\title{
1 Tracking neural activity from the same cells during the entire adult life of mice
}

3 Authors: Siyuan Zhao ${ }^{1,2}$, Xin Tang ${ }^{1,2}$, Sebastian Partarrieu ${ }^{1,2}$, Shiqi Guo ${ }^{1}$, Ren Liu ${ }^{1}$, Jaeyong Lee ${ }^{1}$,

$4 \quad$ Zuwan $\operatorname{Lin}^{1}$ and Jia Liu ${ }^{1, *}$

5

6 Affiliations:

7 1 John A. Paulson School of Engineering and Applied Sciences, Harvard University, Boston, MA 802134 USA

$9 \quad{ }^{2}$ These authors contributed equally to this work: Siyuan Zhao, Xin Tang, Sebastian Partarrieu

10 *e-mail: jia_liu@seas.harvard.edu 


\section{Abstract}

13 Recording the activity of the same neurons over the adult life of an animal is important to

14 neuroscience research and biomedical applications. Current implantable devices cannot provide

15 stable recording on this time scale. Here, we introduce a method to precisely implant

16 nanoelectronics with an open, unfolded mesh structure across multiple brain regions in the mouse.

17 The open mesh structure forms a stable interwoven structure with the neural network, preventing

18 probe drifting and showing no immune response and neuron loss during the yearlong implantation.

19 Using the implanted nanoelectronics, we can track single-unit action potentials from the same

20 neurons over the entire adult life of mice. Leveraging the stable recordings, we build machine

21 learning algorithms that enable automated spike sorting, noise rejection, stability validation, and

22 generate pseudotime analysis, revealing aging-associated evolution of the single-neuron activities. 
24 Long-term stable recording ${ }^{1-4}$ of the same neuron at single-cell and single-spike resolution over

25 the entire adult stage of life of behaving animals is important to understand how neural activity

26 changes with learning and age $e^{4,5,6,7}$, to improve current brain-machine interface performance by

27 reliably interpreting the brain's behavioral and internal states ${ }^{4,6}$, and to study neurodegenerative

28 diseases, aging-associated neurological disorders and cognitive decline ${ }^{7,8}$. Current implantable

29 electronic and optical tools can record neural activity at single-cell and single-spike resolution but

30 suffer from immune response and recording drift due to the mechanical and structural disparities

31 between rigid electronic or optical devices and brain tissue $\mathrm{e}^{9,10}$. Relative shear and repeat motion at

32 the implanted interface keep changing the relative position between recording devices and

33 recorded neurons. The proliferation of astrocytes and microglia form a $\sim 100 \mu \mathrm{m}$ thick glial sheath

34 that cause the death of neurons and isolate recording devices from neurons. Together, they lead to

35 chronic instability of recordings. Optical imaging techniques are further limited by the light

36 penetration depth and three-dimensional (3D) volumetric scanning across the $3 \mathrm{D}$ tissue due to

37 optical aberration and attenuation ${ }^{11}$.

Miniaturized flexible electronics such as mesh nanoelectronics and thin-film probes have been utilized in in vivo electrophysiology ${ }^{12-17}$ given their unique mechanical properties. Mesh nanoelectronics provide a chronically stable, gliosis-free implantation over a few months through the incorporation of tissue-like structural and mechanical properties into nanoelectronics ${ }^{18-20}$. However, due to their mechanical flexibility, invasive methods such as syringe injection are required to implant the tissue-like electronics into the brain ${ }^{18-20}$. The relatively large mechanical damage from the implantation causes permanent damage to the neural network. In addition, implanted mesh nanoelectronics can only unfold in the cavities of the brain such as the 
46 subventricular zone, not in tissue-dense brain regions ${ }^{18-21}$. As a result, the bundled mesh

47 nanoelectronics do not have the optimized mechanical flexibility required to interface with the

48 brain tissue over long time periods ${ }^{18-21}$. On the other hand, flexible thin film brain probes lack the

49 open mesh structures that allow for $3 \mathrm{D}$ integration with the neural network ${ }^{22-24}$. They also need to

50 maintain mechanical strength to prevent damage of the probe during the implantation ${ }^{22-25}$, which

51 can cause an immune response and probe drifting during long-term implantation. As a result, none

52 of the existing technologies has demonstrated long-term stable tracking of the same neuron over

53 the entire adult stage of life of a behaving animal.

54 Here, we solved this issue by implanting a fully unfolded tissue-like mesh nanoelectronics into the

55 brain of a mouse. The fully unfolded mesh can form an interwoven structure within the neural

56 network and eliminate the immune response and probe drifting, maintaining a long-term stable

57 electrode-to-neuron interface at the single-cell level, thus enabling the same neuron to be recorded

58 over the entire adult life of animals (Fig. 1a). To enable this method, we developed mesh

59 nanoelectronics monolithically integrated with ultra-thin and releasable polymer shuttles through

60 lithographic fabrication. We also incorporated unique polymer anchors and water-releasable

61 structures, allowing for controllable, precise, and minimally invasive delivery of mesh

62 nanoelectronics in the mouse brain. This implantation method can keep the designed open mesh

63 structure of the device in the brain across multiple brain regions, including cell-dense regions. The

64 open mesh allows the neural network to form seamlessly interwoven structures, provides a tissue-

65 level flexible interface, and prevents the repeated micromotion and drift between recording

66 electrodes and surrounding neural tissue during yearlong recording, thus allowing for a highly

67 stable recording of neuron activities across multiple brain regions. 
68 By optimizing the size of the implanted nanoelectronics, we achieved stable tracking of the same

69

70

71

72

73

74

75

76

77

78

79

80

81

82

83

84

85

86

87

88

89 neuron over the entire adult life of mice until their natural death (i.e., 5-18 months for mouse \#1, 5-20 months for mouse \#2, and 5-19 months for mouse \#3), confirmed by vigorous statistical tests $^{3,18-21,26}$. Tracking the whisker-stimulation-evoked single spikes from the barrel cortex indicated that the electrode embedded in a single whisker barrel does not drift over the animal's adult life. Using the first several months' recording data to train an autoencoder ${ }^{27}$, a machine learning (ML) tool for representation learning, we further confirmed the stable recording, which also allowed for fully automatic spike sorting, noise rejection, and stability analysis over the adult life of the mice. Finally, ML-based pseudotime analysis of single-unit waveforms identified several neurons with age-dependent changes in electrical activities.

\section{Results}

\section{Monolithically integrated mesh nanoelectronics and ultra-thin shuttle}

To implant completely open and unfolded mesh nanoelectronics into the brain, we integrated the mesh nanoelectronics monolithically with a releasable, ultra-thin polymeric shuttle using standard photolithography procedures (Fig. 1b, Extended Data Fig. 1, 2a-k, Methods). The mesh nanoelectronics were fabricated as described in previous reports ${ }^{18-20}$. Briefly, 16 or $3215-\mu \mathrm{m}-$ diameter electrodes were connected by SU-8 encapsulated $\mathrm{Cr} / \mathrm{Au}$ interconnects to $\mathrm{Cr} / \mathrm{Au}$ input/output (I/O) pads. The encapsulated interconnects were $10-\mu \mathrm{m}$ wide and $<1-\mu \mathrm{m}$ thick, forming a mesh network with a 2D filling ratio at $73.3 \%$, which yielded an effective bending stiffness of $1.26 \times 10^{-15} \mathrm{~N} \cdot \mathrm{m}^{2}$. The mesh nanoelectronics were partially fabricated on the top of a Ni sacrificial layer. Next, a $25-\mu$ m-thick polymer shuttle was defined on the top of the mesh 
nanoelectronics with a 3 - $\mu$ m-thick water-soluble dextran and $20-\mu \mathrm{m}$-thick polymer anchors. These polymer anchors (Fig. 1b, inset) connected the mesh nanoelectronics with the polymer shuttle

92 through the dextran layer (Fig. 1b, red box). After the integrated device was released from the

93 substrate (Fig. 1c, top) by removing the Ni sacrificial layer, the anchor kept the pattern of the mesh nanoelectronics on the polymer shuttle. Then, a few drops of $10 \mathrm{wt} \%$ PEG (35 kDa) was coated to reinforce the bonding between the mesh nanoelectronics and polymer shuttle (Fig. 1c, bottom), as well as enhanced the temporary stiffness and provided protection to the mesh nanoelectronics

97 during implantation. The biodegradable PEG adhesion layer has a sub-micron thickness; thus, the surgical footprint is mostly affected by the thin polymer shuttle. After removing the anchor connection (Fig. 1c, bottom), the mesh nanoelectronics can be readily released from the polymer shuttle by dissolving the PEG in an aqueous solution (Fig. 1d).

The polymer shuttle was used to guide the implantation of the mesh nanoelectronics into the brain tissue (Fig. 1e, left). After the integrated brain probe reached the targeted position, saline was applied to quickly dissolve the PEG and to release the mesh nanoelectronics from the polymer shuttle, which was subsequently withdrawn from the brain tissue (Fig. 1e, right). We characterized the implantation procedure in a transparent $0.6 \mathrm{wt} \%$ agarose gel-based brain phantom with mechanical properties comparable to that of the brain tissue ${ }^{28}$. At a typical implantation speed of $100 \mu \mathrm{m} / \mathrm{s}$, we did not observe any buckles on the probes (Fig. 1f, left). After insertion, 1× phosphate buffered saline (PBS) solution was applied to dissolve the PEG/dextran adhesive layer. The shuttle was then withdrawn at a speed of $10 \mu \mathrm{m} / \mathrm{s}$. After withdrawing the shuttle, the mesh nanoelectronics still maintained its implantation location and open mesh structure without any

111 deformations (Fig. 1f, right). We tested the yield of the implantation in brain phantoms with 112 various speeds. We achieved a 93.3\% yield for insertion and extraction of 16-channel mesh 
nanoelectronics at $100 \mu \mathrm{m} / \mathrm{s}$ (Extended Data Fig. 21, m). There was no significant change of implantation yield with different size mesh electronics of 32 channels $(n=3, p>0.05$, Extended Data Fig. 21, m).

The optimized implantation procedure was then used for mouse brain implantation. Figure $1 \mathrm{~g}$ shows typical implantation of 300- $\mu$ m-wide 16-channel mesh nanoelectronics in the anesthetized mouse brain (Methods). The probe (Fig. 1g, inset) can be easily implanted with the polymer shuttle withdrawn by the same conditions tested for the phantom gel. Damages to the blood vessels were minimal throughout the imaging-guided implantation. To evaluate acute tissue damage, cell loss, and mesh nanoelectronics distribution, we imaged the post hoc fixed and stained tissue slices immediately after implantation (Fig. 1h). Acute damaged area with the thin-shuttle was approximately $0.0068 \pm 0.0016 \mathrm{~mm}^{2}$ (mean $\pm \mathrm{SD}, n=5$, Fig. $1 \mathrm{~h}$ ), which is significantly smaller than those from previous reported implantations using syringe-injection ${ }^{18,19}\left(0.0164 \pm 0.0033 \mathrm{~mm}^{2}\right.$, mean $\pm \mathrm{SD}, p<0.01, n=5$, Fig. $1 \mathrm{~h})$ and biodegradable shuttles ${ }^{21,29}\left(0.0182 \pm 0.0039 \mathrm{~mm}^{2}\right.$, mean $\pm \mathrm{SD}, p<0.01, n=5$, Fig. $1 \mathrm{~h})$. While the tissue damage showed no significant difference compared with samples implanted by $50-\mu \mathrm{m}$ diameter tungsten wire ${ }^{25}\left(0.0062 \pm 0.0014 \mathrm{~mm}^{2}\right.$, mean $\pm \mathrm{SD}, n$ $=5$, Fig. 1h), the cross-sectional images of brain slices with implants showed the clear unfolded mesh structures vs. bundled ribbons by tungsten probe-based delivery (Fig. 1i). Compared with a previously demonstrated implantation method for flexible neural probes ${ }^{18-21,25,29,30}$, the integrated $25-\mu m$-thick polymer shuttle drastically reduces tissue displacement during implantation as well as maintains the designed open structure with nearly $90 \%$ implantation yield. Moreover, the presented method involves minimal manual manipulations since the ultra-flexible nanoelectronics was pre-attached to the thin-shuttle with the lithography process. On average, it took less than 3 min to assemble one mesh nanoelectronics (more than 20 nanoelectronics per hour), including sub- 
micro-thick PEG coating, anchor dicing, and additional packaging with a success rate approaching $100 \%$.

\section{Unfolded mesh nanoelectronics structure 3D interwoven with the neural network.}

We implanted mesh nanoelectronics with different sizes across multiple brain regions. Each mesh structure has an ultra-small cross-section of $10 \times 1 \mu \mathrm{m}^{2}$. The longitudinal bending stiffness of each individual mesh structure reached $1.26 \times 10^{-15} \mathrm{~N} \cdot \mathrm{m}^{2}$, which is comparable to that of brain tissue and orders of magnitude lower than state-of-the-art probes (i.e., ultrasmall carbon ${ }^{31-33}$, polyimide ${ }^{34}$ and elastomer-based 'e-dura' probes ${ }^{35}$ ). Rhodamine $6 \mathrm{G}$ was added to the SU-8 encapsulation layer, enabling the imaging of the mesh structure in the brain. To explore the potential capability of the implantation, Figure 2a shows a 2-mm-wide, 3-mm-long mesh nanoelectronics implanted into a mouse brain across cortex, hippocampus, and thalamus regions. The size of this device can potentially include 1,024 recording sites through 3D stacking of electrodes ${ }^{36}$ (Fig. 2a-d, Methods). A representative $3 \mathrm{D}$ reconstructed image of the mesh nanoelectronics in the brain tissue at 6-week post-implantation (Fig. 2a) showed the fully unfolded, open mesh structure interweaving with neurons and astrocytes across multiple brain regions (i.e., cortex, hippocampus, thalamus, etc.). A slight bending of the mesh suggested that the tissue-like nanoelectronics were flexible within the tissue. A zoomed-in view of the hippocampus CA1 region (Fig. 2b) shows a smooth distribution of neurons and astrocytes across the mesh. Notably, neurons in the cell-dense region (hippocampus) can still penetrate the open mesh structure (Fig. 2c), forming an intertwined tissue-nanoelectronics interface. Figure $2 \mathrm{~d}$ shows that the size of the recording electrode (white dashed circles) is comparable to the size of the soma. The subcellular feature size, tissue-level flexibility, and 3D interwoven network collectively eliminated the micromotion between the functional electrode and 
recorded neurons ${ }^{37}$. Additional replications of mesh nanoelectronics with different sizes were implanted in mouse brains and are shown in Extended Data Fig. 3.

Next, we performed longitudinal immunostaining characterizations to assess the distribution of key cell types around mesh nanoelectronics over the time course of implantation. To demonstrate that the open mesh structure reduces immune responses during chronic implantation (Fig. 2e, top), thin-film nanoelectronics with the same dimensions as mesh nanoelectronics were used (Fig. 2e, bottom) as control and contralaterally implanted in the same mouse brain. The bending stiffness of the thin film nanoelectronics is only slightly higher than that of the mesh nanoelectronics $(39.8 \times$ $10^{-15} \mathrm{~N} \cdot \mathrm{m}^{2}$ vs. $1.26 \times 10^{-15} \mathrm{~N} \cdot \mathrm{m}^{2}$, Methods). The brain tissue was harvested and sliced for immunostaining 2-, 6-and 12-week, and 1-year post-implantation. Horizontal slices were stained with cell-type-specific protein markers for imaging of neurons, astrocytes, and microglia (Extended Data Fig. 4). We quantitatively analyzed horizontal brain slices implanted with 16channel, 300- $\mu \mathrm{m}$-wide film/mesh nanoelectronics (Fig. 2f-i, Methods). Protein marker signals were calculated by normalizing the fluorescence intensity around the implantation site with the baseline value defined as the average fluorescence intensity over a range of 525-550 $\mu \mathrm{m}$ away from the nanoelectronics. Statistical analysis demonstrated a significant degradation of neuron density (NeuN) and an enhancement of astrocytes and microglia intensity (GFAP and Iba-1, respectively) near the thin-film nanoelectronics at all time points ( $p<0.05, n=5$, Fig. $2 \mathrm{f})$. These results proved that the thin-film nanoelectronics can still trigger the proliferation of astrocytes/microglia and reduced the neuron density at the nanoelectronics-brain interface. Meanwhile, the open mesh nanoelectronics introduced minimal damage to the surrounding neurons and negligible immune response. Importantly, the result demonstrated that no significant neuron loss was detected at 2-week post-implantation for mesh samples (Fig. 2f), suggesting 
minimal acute damage from the thin-polymer shuttle. In addition, the neuron density near the mesh surface remained the same at one-year post-implantation (Fig. 2f).

We further calculated the normalized intensity of neural cell fluorescence signals within regions 100- $\mu \mathrm{m}$ away from the nanoelectronics to assess neuron loss and inflammation reaction at the different post-implantation periods (Fig. 2g-i). The mesh nanoelectronics samples showed a neuron density of $83.9 \pm 13.0 \%$ (mean $\pm \mathrm{SD}, n=5$, Fig. $2 \mathrm{~g})$ at 2 weeks, which is greater $(p<0.001$ $n=5$, Fig. $2 \mathrm{~g})$ than that from the thin film nanoelectronics $(66.9 \pm 7.8 \%$, mean $\pm \mathrm{SD}$, Fig. $2 \mathrm{~g})$ in the contralateral brain slices. Compared to the non-implanted regions, neuron intensity of mesh nanoelectronics samples increased to $93.1 \pm 10.2 \%, 99.1 \pm 8.0 \%$, and $102.4 \pm 11.2 \%$ (mean $\pm \mathrm{SD}$, $n=5$, Fig. 2g) 6 weeks, 12 weeks, and 1-year after implantation, respectively. On the contrary, thin-film nanoelectronics samples showed significant neuron loss for the same periods $(70.2 \pm$ $4.6 \%, 84.8 \pm 3.3 \%$, and $85.2 \pm 2.3 \%$ at 6 weeks, 12 weeks, and 1-year after implantation, respectively. $p<0.05, n=5$, Fig. $2 \mathrm{~g}$ ). The intensity of astrocytes and microglia around the mesh slightly increased at 2 weeks $(115.1 \pm 9.1 \%$ at microglia, $132.6 \pm 20.5 \%$ at astrocytes, mean $\pm \mathrm{SD}$, $n=5$, Fig. $2 \mathrm{~h}, \mathrm{i})$ and then reduced at 6 weeks $(113.6 \pm 5.8 \%$ at microglia, $110.1 \pm 10.0 \%$ at astrocytes, mean $\pm \mathrm{SD}, n=5$, Fig. 2h, i). Moreover, continuous monitoring of the inflammation around the mesh nanoelectronics revealed nearly normal immune cell distribution at 12 weeks $(95.3 \pm 13.1 \%$ at microglia, $105.5 \pm 12.7 \%$ at astrocytes, mean $\pm \mathrm{SD}, n=5$, Fig. $2 \mathrm{~h}$, i), and even up to one year $(104.9 \pm 9.0 \%$ at microglia, $109.5 \pm 17.9 \%$ at astrocytes, mean $\pm \mathrm{SD}, n=5$, Fig. $2 \mathrm{~h}$, i). We attribute the little-to-no immune response of the mesh nanoelectronics to the ultra-flexible open structure that is imperceptive to surrounding brain tissue, neurons, and the cells involved in inflammation. Compared with the open mesh structure, the thin-film nanoelectronics implantation introduced significantly higher levels of astrocytes $(181.9 \pm 17.9 \%, 272.6 \pm 40.3 \%, 174.4 \pm 22.8 \%$ 
and $182.1 \pm 32.7 \%$ at 2-, 6-, 12-and 1-year post-implantation, respectively, mean $\pm \mathrm{SD}, n=5$, Fig. $2 \mathrm{~h}, \mathrm{i})$ and microglia aggregation $(177.2 \pm 14.5 \%, 162.3 \pm 18.4 \%$, and $125.1 \pm 12.6 \%$ and $154.9 \pm$ $30.0 \%$ at 2-, 6-, 12-and 1-year post-implantation, respectively. Mean $\pm \mathrm{SD}, n=5$, Fig. 2h, i) over the same period $(p<0.05$, significant enhancement compared with the open mesh at all time points, Fig. 2h, i). Notably, we can still observe the proliferation of astrocytes and microglia around the thin-film nanoelectronics at one year post-implantation. Together, these results demonstrate that open mesh nanoelectronics introduce little-to-no inflammation and mechanical damage to the surrounding tissues as compared with thin-film nanoelectronics over yearlong implantation.

\section{Long-term stable recording at single-cell resolution across multiple brain regions.}

To test the stability of the recording, we implanted $600-\mu \mathrm{m}$-wide mesh nanoelectronics with 32 channels and 300- $\mu$ m-wide mesh nanoelectronics with 16 channels into multiple mouse brain regions for head-fixed behaving electrophysiology (Methods). Electrodes were implanted into different brain regions including the somatosensory cortex and striatum (32-channel, mesh nanoelectronics\#1, Fig. 3a); red nucleus, interstitial nucleus, and ventral tegmental area in midbrain (32-channel mesh nanoelectronics\#2, Fig. 3a); and visual cortex and hippocampus (16channel mesh nanoelectronics\#3, Fig. 3a). Putative individual neurons were isolated using Waveclus $3^{38}$ (Methods). Intrinsic spike waveform variability from the superficial (mesh nanoelectronics \#1, \#3, Fig. 3b, d) and deep (mesh nanoelectronics \#2, Fig. 3c) brain regions is consistent with different putative neuron types in each brain region ${ }^{39,40}$. Moreover, the hippocampus recordings show higher neuron yield per electrode $(2.0 \pm 0.4$ neurons, median \pm 1.5 interquartile range, $n=8$, Fig. $3 \mathrm{e})$, spike amplitude $(148.146 \pm 77.4 \mu \mathrm{V}$, median \pm 1.5 interquartile range, $n=8$, Fig. 3f) and firing rate $(17.3 \pm 7.6$ spike/s, median \pm 1.5 interquartile range, $n=8$, 
Fig. 3g) as compared to other recorded brain regions (primary somatosensory cortex, striatum, midbrain, primary visual cortex, Fig. 3e-g).

Next, we evaluated the long-term stability of recordings from 32-channel and 16-channel mesh nanoelectronics from 5 independent animals ( $n=43$ electrodes from two 32-channel and $n=29$ electrodes from three 16-channel mesh nanoelectronics). 72 putative individual neurons from multiple regions were recorded 10 days post-implantation, which increased to 115 putative individual neurons after 60 days. Both 16-channel and 32-channel mesh nanoelectronics show low noise level and high signal to noise ratio (SNR) at 60 days post-implantation in behaving animals (16-channel: $9.97 \pm 1.72 \mu \mathrm{V}$ at noise level, $12.67 \pm 6.34$ at SNR, $n=29$ electrodes; 32-channel: $9.27 \pm 2.10 \mu \mathrm{V}$ at noise level, $13.25 \pm 5.94$ at $\mathrm{SNR}, n=43$ electrodes, mean $\pm \mathrm{SD})$. The statistical results (Fig. 3h-j) showed that the normalized neuron count per electrode, average amplitude, and SNR of $300-\mu \mathrm{m}$-wide,16-channel mesh nanoelectronics increased over the first 30 days of implantation and then stabilized ( $n=29$ electrodes from three 16-channel mesh nanoelectronics).

These parameters from the 600 - $\mu \mathrm{m}$-wide, 32 -channel mesh nanoelectronics stabilized at 50 -day post-implantation, suggesting the potential device size-related effect on the signal stability $(n=43$ electrodes from two 32-channel mesh nanoelectronics). These results contrast with reports from previous brain probes where amplitudes, SNR, and neuron counts degrade weeks after implantation ${ }^{3,437}$, suggesting that the unfolded, open mesh nanoelectronics formed a long-term stable interface with neurons and tissue.

\section{Tracking the same neuron's activity over the entire adult life of mice}


Two mice implanted with 16-channel mesh nanoelectronics and one mouse with 32-channel mesh nanoelectronics were recorded monthly until their natural death (5-18 months for mouse \#1, 5-20 months for mouse \#2, and 5-19 months for mouse \#3). $82.8 \pm 6.2 \%$ neurons are stably recorded

252 (mean $\pm \mathrm{SD}$, compared to the first recording session). Compared to mice of 5 months with glossy brown fur, the aged mice of 18-months with mesh electronics implanted exhibited normal and

254 healthy aging, including weight gain, barbering around the eyes, and thinning and grey fur in the dorsal back $\operatorname{skin}^{41}$ (Extended Data Fig. 5a-e). Statistical analysis revealed that there was a significant increase of gray hairs and a decrease of black hairs in aged mice when compared to the mature adult mice ( $p<0.05, n=3$, Extended Data Fig. 5f). The electrode interfacial impedances exhibited relatively constant values of $920.2 \pm 107.2 \mathrm{k} \Omega$ vs. $857.2 \pm 85.7 \mathrm{k} \Omega$ at months 6 vs. 18

259 (mean $\pm \mathrm{SD}, n=30$, Extended Data Fig. 6a), indicating stable electrical and mechanical properties of the mesh nanoelectronics ${ }^{42}$.

We first assessed the stability of the signals by spike sorting and statistical analyses. Spike waveforms were projected to a $2 \mathrm{D}$ embedding space for stability validation by using UMAP- ${ }^{26}$ and PCA-based dimension reduction algorithms. PCA is commonly used to define the number and stability of recorded single-neuron signals over time ${ }^{19,20,31}$. UMAP is a non-linear ML-based dimension reduction algorithm that can learn a low-dimensional embedding space to preserve as much of the local and more of the global data structure than linear dimension reduction algorithms

267 such as PCA. 26 neurons were isolated across all recording sessions starting from 5 months and lasting until natural death. The clusters for each sorted spike in both UMAP and PCA embeddings show nearly constant positions and well separated from each other in the first and second 270 component plane (UMAP1-UMAP2 and PC1-PC2) through >1-year recordings (Fig. 4a, Extended 271 Data Fig. 6b, Methods). In addition, the corresponding single-unit waveforms' shapes (Fig. 4b), 
as well as their firing dynamics (i.e., interspike interval) were stable (Extended Data Fig. 6c, Methods). Auto-correlation analysis showed that single-unit waveforms were highly similar and almost indistinguishable to themselves $(0.90 \pm 0.14$ across all recording sessions from 3 mice, mean $\pm \mathrm{SD}$, Fig 4c, Methods). L-ratio ${ }^{43}$ and silhouette score ${ }^{44}$ analysis (Fig. 4d, Methods) confirmed good unit separation and accurate identification of individual neurons, demonstrating that the signals were sufficiently separated to permit isolation of single units. Statistical analysis on single-unit recording stability examined by five waveform features (amplitude, duration, peaktrough ratio, repolarization slope, and recovery slope $)^{40}$ and signal-to-noise ratio (SNR) showed that their average values were nearly constant and the majority ( $79 \%$ from 3 mice) of neuron waveform features did not change significantly over time $(p>0.05$ two-sided $t$ test, Fig. 4e-h, Methods), demonstrating that the neuron spikes showed consistent characteristic features over time. Notably, the consistent signal-to-noise ratio (SNR) demonstrated that the electrode-to-cell interface was not degraded during the entire period (Fig. 4f). Collectively, all these results indicate that the waveforms were stably recorded from the same neuron over the entire recording period.

In addition to recording of spontaneous activity, we validated the stability of recording by examining the stable recording of whisker stimulation-elicited neuron activities ${ }^{32,45}$. Specifically, we identified one electrode (electrode A) on the mesh nanoelectronics as being close to a D2 barrel neuron in primary somatosensory cortex ( $\mathrm{S} 1$ cortex) by successful recording of the whiskerstimulation-elicited single-unit spikes (Fig. 4i-1). Recording from stimulation of other whiskers (e.g., C3) or another electrode (e.g., electrode B that is close to electrode A) away from the D2 barrel field were used as control (Extended Data Fig. 7). We performed the whisker deflection with a galvanometer-driven stimulation contralateral to the implant (Fig. 4i). A $1 \mathrm{~Hz}, 900 \mathrm{deg} / \mathrm{s}$ deflection was applied to the targeted whisker (Methods). The raster plot and peri-stimulus time 
histogram (PSTH) of this single-unit recording showed that the electrode $\mathrm{A}$ of the mesh nanoelectronics can record strong and rapid neuron firing in response to the principal whisker D2 deflection (Fig. 4j). We can record well defined neuron activity and waveforms from 8 months to 16 months (Fig. 4j). Spikes observed from the electrode A with C3 whisker deflection (Extended Data Fig. 7a-c) or from electrode B with D2 whisker stimulation (Extended Data Fig. 7d-e) showed no correlation with the whisker stimulation. Notably, Figure $4 \mathrm{k}$ and 1 showed that the evoked spike count and the time delay to the stimulation exhibited no significant change over time $(p>0.05, n$

$302=5$ ). These behavior-triggered electrical activities further demonstrated the capability of this method to track the same neuron during the adult lifetime of mice.

\section{ML-based validation and analysis}

306 We further applied an unbiased, autoencoder-based ${ }^{27}$ automated neuronal signal processing and 307 analysis to benchmark the stability of the signal. Autoencoders is a self-supervised learning 308 algorithm, which is only able to meaningfully reconstruct data similar to what they have been trained on, thus providing an unbiased way to examine the stability of the recording. We trained three two-headed autoencoders by using detected spike waveforms, corresponding electrode

311 information and sorted neuron labels from the first 6-month recording data of the three mice. Each

312 autoencoder i) learned nonlinear dimension reduction transformations compressing spike 313 waveforms into a 2D embedding space, ii) classified the spike as a specific neuron in the training 314 data, and iii) reconstructed the embeddings back to the input data space (Fig. 5a). The classification 315 and reconstruction were simultaneously optimized during the training of the autoencoder, enabling 316 the autoencoder the capabilities of spike sorting, postprocessing, and stability verification

317 (Extended Data Fig. 8a, Methods) at the same time. Notably, the autoencoder showed much faster 
classification speed and higher reconstruction accuracy compared with UMAP and random forest ${ }^{46}$ classifier-based spike sorting (Extended Data Fig. 8b, Methods). While we used the autoencoder trained with the first six-month recording to achieve the best performance, we found that a twomonth recording dataset is sufficient to train the model with only about $4 \%$ accuracy decrease (Extended Data Fig. 8c). The waveforms of the remaining 8-month recording data can still be classified and reconstructed (Fig. 5b and Extended Data Fig. 8d) with the classification accuracy (Fig. 5c, Extended Data Fig. 8e) reaching $89 \pm 4 \%$ (mean $\pm \mathrm{SD}, n=3$ mice). An anomaly dataset was constructed to simulate the drift of the recording using spikes gathered from a fourth independent mouse to test the drift detection ability of the autoencoder. The results showed that the mean squared error (MSE) between reconstructed and original waveforms (Fig. 5d) was higher for spikes detected from simulated drifting neurons, compared to the stable neurons used in the training and testing dataset. This significant difference allowed for drift detection based on reconstruction accuracy. A threshold could be used to distinguish the testing spikes and drifting spikes, which eliminated the majority of spikes from the drifting dataset $(83 \pm 12 \%$, mean $\pm \mathrm{SD}, n$ $=3$ mice) and kept most spikes from the testing dataset $(86 \pm 7 \%$, mean $\pm \mathrm{SD}, n=3$ mice, Extended Data Fig. 8f). By visualizing the autoencoders' embedding space in the bottleneck (Fig. 5e), cluster embeddings of the neuron spike waveforms from the same mouse showed higher separability than drifting spikes from a different mouse (Extended Data Fig. 8g-k). Furthermore, the training manifold convex hull was used as a stability verification tool for spike processing to quantify the within-boundary subset of testing dataset spikes (Fig. 5f and Extended Data Fig. 8h-k). Low amounts $(8 \pm 11 \%$, mean $\pm \mathrm{SD}, n=3$ mice) of out-of-manifold testing spike embeddings further demonstrated recording stability as a similarity in waveform shape between the first and last recording months, meaning the later spikes' embeddings lay within the embedding space created 
341 by the first recorded spikes (Fig. 5f and Extended Data Fig. 8h-k). Collectively, high classification

342 accuracy and low out-of-manifold percentage confirmed the long-term stable single-unit spike

343 detected from the same neurons over time. This result also suggests that the stable recording data

344 can be used to build the ML model to perform automated spike sorting based on the first few

345 months of recording from a given mouse by capturing single-neuron waveform salient

346 characteristics used as the input to the classification head. Additionally, the ML model successfully

347 detected the spikes from a different mouse, highlighting the model's ability to detect the drift of

348 recording.

\section{Entire adult life study of brain aging at the single-neuron level}

351 The adult mice life recording offers an opportunity to observe aging-associated electrical behavior

352 changes at single-neuron resolution. We performed both qualitative and quantitative analyses of 353 aging-associated changes at the single-neuron level over the mouse adult life. Analysis of clusters using PCA showed the stability of spikes from a group of neurons with largely overlapping clusters (purple and red, respectively) from each electrode, while the other spikes (green and blue) from the same electrode showed a slight change over the time course of recording (Fig. 6a). We quantitatively assessed the multivariate spread of cluster centroid positions by comparing average

358 position shifts between consecutive cluster centroid positions (Fig. 6a, Methods) to average cluster

359 distribution spreads. This assessment supported the stability $\left(0.47\right.$ o $\left._{\text {purple, }} 0.58 \mathrm{o}_{\text {red }}\right)$ and variability 360 (1.96 o green, 2.63 ơblue) described previously. We analyzed the time-evolution of average neuron waveforms in a representative 3D feature space while calculating trajectories with a B-spline

362 interpolation of successive positions (Fig. 6b, Methods). Non-correlated features were chosen after 363 performing correlation analysis (duration, peak-trough ratio, and repolarization slope in Extended 
Data Fig. 9a). 21\% of neurons (i.e., green and blue) showed a clear trajectories trend while others analyzed the time-evolution of the UMAP embeddings of spike waveforms (Fig. 6c, Extended Data Fig. 9b) in real time and ML-defined pseudotime ${ }^{47}$. To study the continuous and gradual transition of the neuron waveforms instead of the discrete real time label, we constructed a pseudotemporal path termed as pseudotime to order spikes in the latent space using monocle $3^{47}$, an ML tool originally for exploring the dynamics of gene expression within cell types and trajectories over time (Methods). The pseudotime of stable neurons (red and purple) remained the same value as the real time varied, which further validated the stability in the waveform during the aging-long recording. Similarly, the pseudotime of previously defined slow aging-associated neurons increased as the mouse got old. These qualitative and quantitative results suggest that the longterm stable recording from these open mesh nanoelectronics can track the aging-related electrical activity evolution from the same or same group of neurons in mice at single-cell level.

\section{Conclusion}

379 We demonstrated that the ultra-thin shuttle monolithically integrated mesh nanoelectronics can be implanted across multiple brain regions with an open mesh structure with minimal tissue damage.

381 The open mesh structure is interwoven with the neural network in the brain of the animal, enabling immune response-free implantation and long-term stable 3D electrode-to-neuron integration. This structural stability allowed us to track the activity of the same neuron over the entire adult life of mice until natural death as supported by our extensive statistical data analyses showing stable impedance, waveform, firing dynamics, and recording performance, something not achieved by other state-of-the-art electrodes. We leveraged the high recording stability of this method, capable 
387 of successfully training an autoencoder using the first month's recording, which further validate

388 the stability of recording. Combining the stable recording and autoencoder, we can automate spike

389 processing, sorting and stability verification on the remaining months' recording. The unique

390 ability to successfully track individual neurons in a chronically stable manner over such a long

391 timespan provides a continuous view of aging-associated changes in neural activity. Combining

392 the evolution of spiking activity at both real time and ML-calculated pseudotime, we observed

393 potential aging-associated waveform changes at the single-neuron level. We believe long-term

394 stable tracking of single neuron activity patterns across a stably recorded population of cells

395 combined with automated data analysis tools will open new opportunities for the next-generation

396 brain-machine interface and bioelectronic medicine. This technology also promises to inform our

397 understanding of many long-term processes, including development, learning, recovery from

398 injury, neurodegeneration and age-related cognitive decline. In the future, we envision that further

399 integration of stretchability into our current device design, which can further adapt to the large

400 volume change during early brain development, could further allow us to achieve the long-term

401 stable recording over the entire lifespan of animals. 
bioRxiv preprint doi: https://doi.org/10.1101/2021.10.29.466524; this version posted November 1, 2021. The copyright holder for this preprint (which was not certified by peer review) is the author/funder. All rights reserved. No reuse allowed without permission.

\section{Figures and Figure Legends}

a

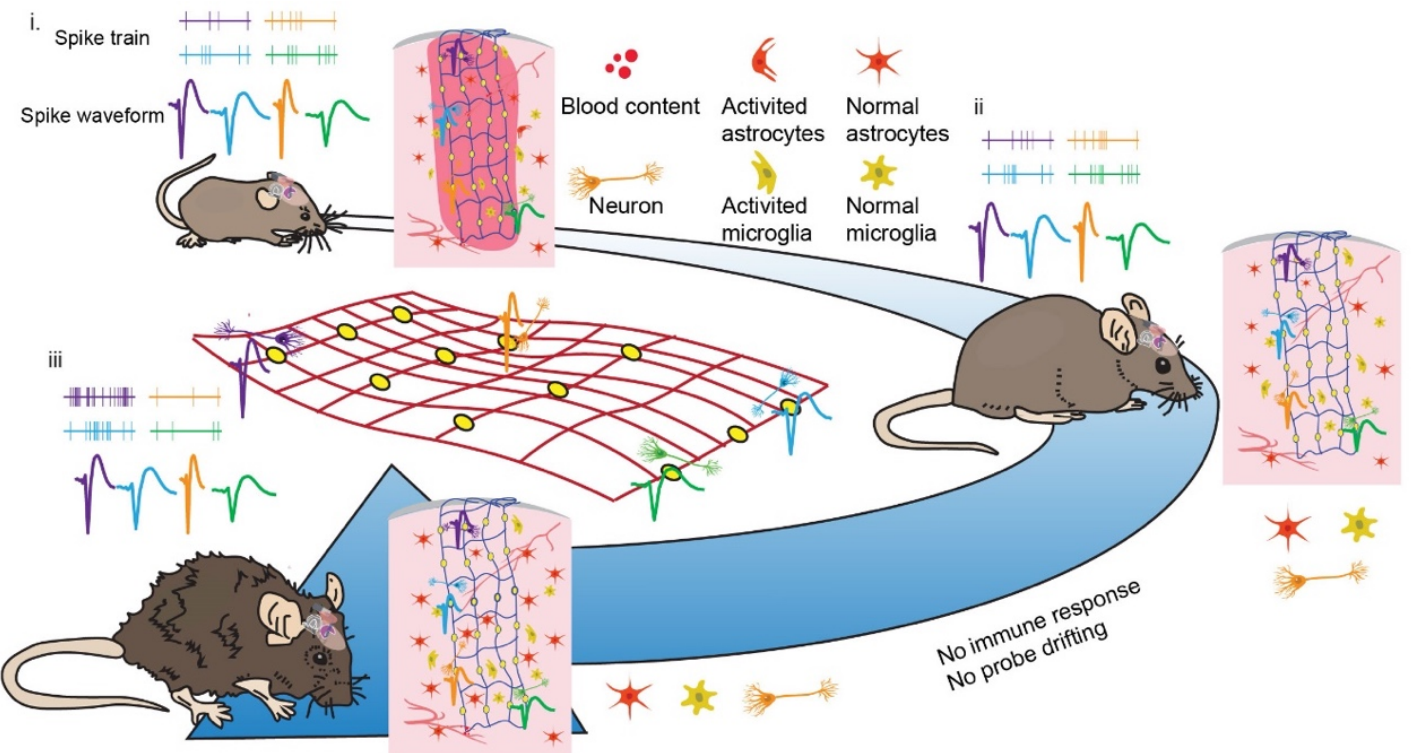

b
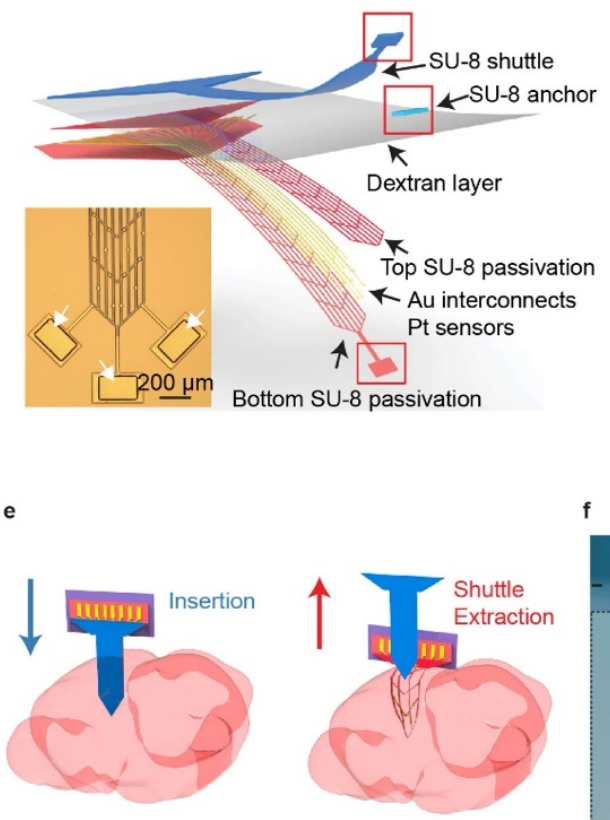

g

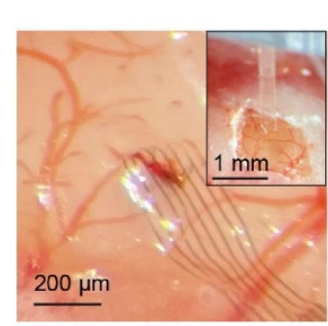

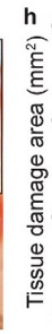

c

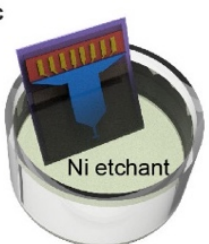

i. I/O pad cleaning and ponding

iii. PEG dip

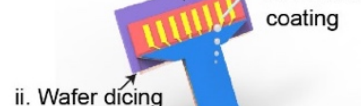

ii. Wafer dícing
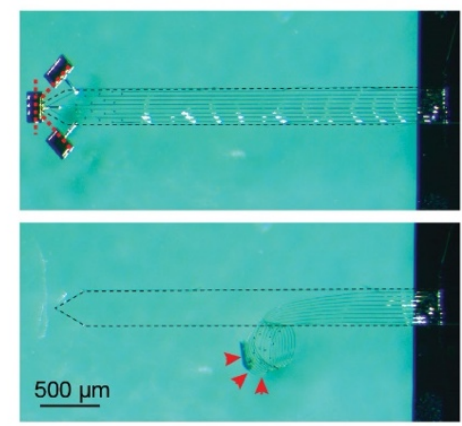

iv. Anchor cutting f
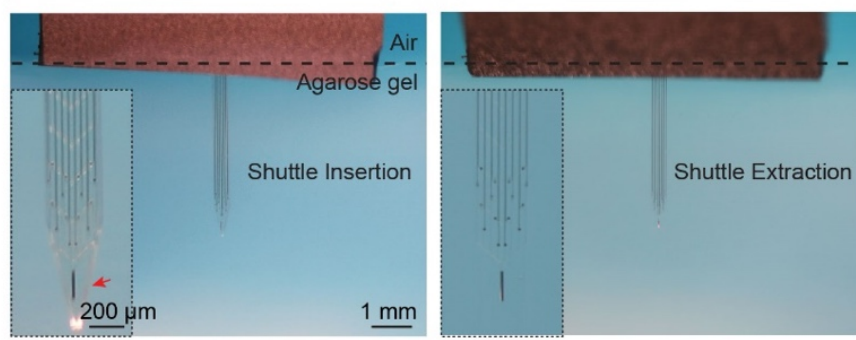
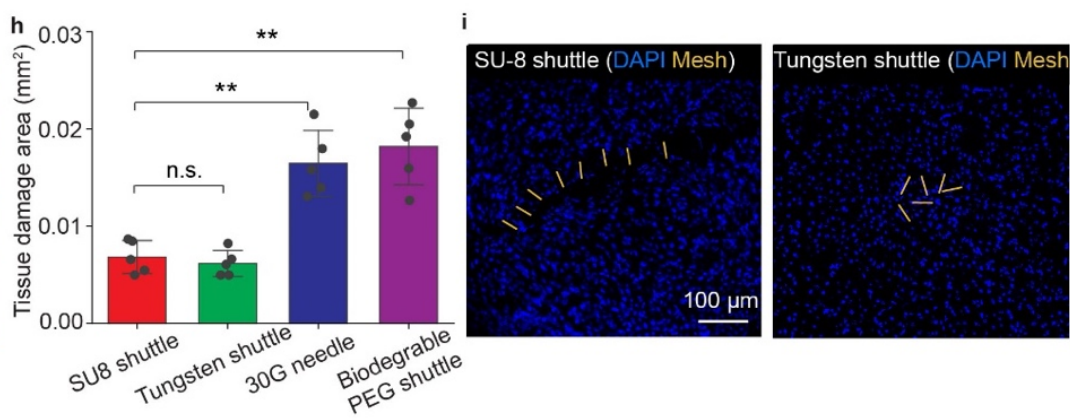
Fig. 1 | Minimally invasive implantation of tissue-level flexible mesh nanoelectronics in the brain. a, Schematics showing the long-term stable electrical recording of the same neuron over the entire adult life of mice enabled by the minimally invasively implanted and fully unfolded tissue-level flexible mesh nanoelectronics. Mesh nanoelectronics seamlessly integrate with neural networks without immune response. Single-cell electrophysiology from the same animal at (i) mature adult (3-6 months), (ii) middle (10-14 months), and (iii) old (18-24 months) stages are recorded. Colored waveforms represent different neurons that are stably recorded over the entire adult life of mice. b, Exploded view of the integrated mesh nanoelectronics showing the distinct material layers. The fully assembled nanoelectronics consisting of (from top to bottom) a 25- $\mu \mathrm{m}$ thick polymer shuttle, a $20-\mu \mathrm{m}$-thick polymer anchor, a 3- $\mu \mathrm{m}$-thick dextran dissolvable layer, a 450-nm-thick top SU-8 encapsulation layer, 50-nm-thick platinum electrodes and 70-nm-thick gold $(\mathrm{Au})$ interconnects, and a 450-nm-thick bottom SU-8 encapsulation layer. Inset: bright-field (BF) microscopic image of mesh nanoelectronics connected with polymer shuttle through anchors (white arrows). c, Schematics showing stepwise releasing of mesh nanoelectronics from the substrate and shuttle. Mesh nanoelectronics was released from the fabrication substrate after removing the Ni sacrificial layer while connected with the polymer shuttle by the anchors (top). The released shuttle/nanoelectronics were cleaned for bonding and dicing, and then coated by the biodegradable PEG through dip-coating. After cutting the polymer anchors, the mesh nanoelectronics was released from the shuttle by dissolving PEG (bottom). d, Photographs showing the released polymer shuttle/mesh nanoelectronics hybrid from the substrate (top) and released mesh nanoelectronics from the shuttle (bottom). Red dashed lines and arrows highlight the cutting lines of the anchors and the released mesh nanoelectronics, respectively. $\mathbf{e}$, Schematics showing the brain implantation process. f, In vitro images of mesh nanoelectronics implantation in a $0.6 \%$ agarose gel. Insets: zoom-in images showing the released mesh nanoelectronics maintain the unfolded structure and implantation location after withdrawing the polymer shuttle. $\mathbf{g}$, Photograph showing the representative brain implantation with minimal tissue damage. Inset: ultrathin polymer shuttle-enabled implantation. $\mathbf{h}$, Statistical analysis of tissue acute damage zone of different implantation methods. Data represented as mean $\pm \mathrm{SD}$, individual data points are overlaid ( ${ }^{*} p<0.01$, two-tailed unpaired $t$ test, $n=5$ ). i, Representative images of 20 - $\mu$ m-thick horizontal brain slices showing the acute mechanical injuries by the ultrathin-polymer shuttle (left) and 50- $\mu \mathrm{m}$ diameter tungsten shuttle (right). Yellow and blue represent mesh nanoelectronics and DAPI, respectively. 

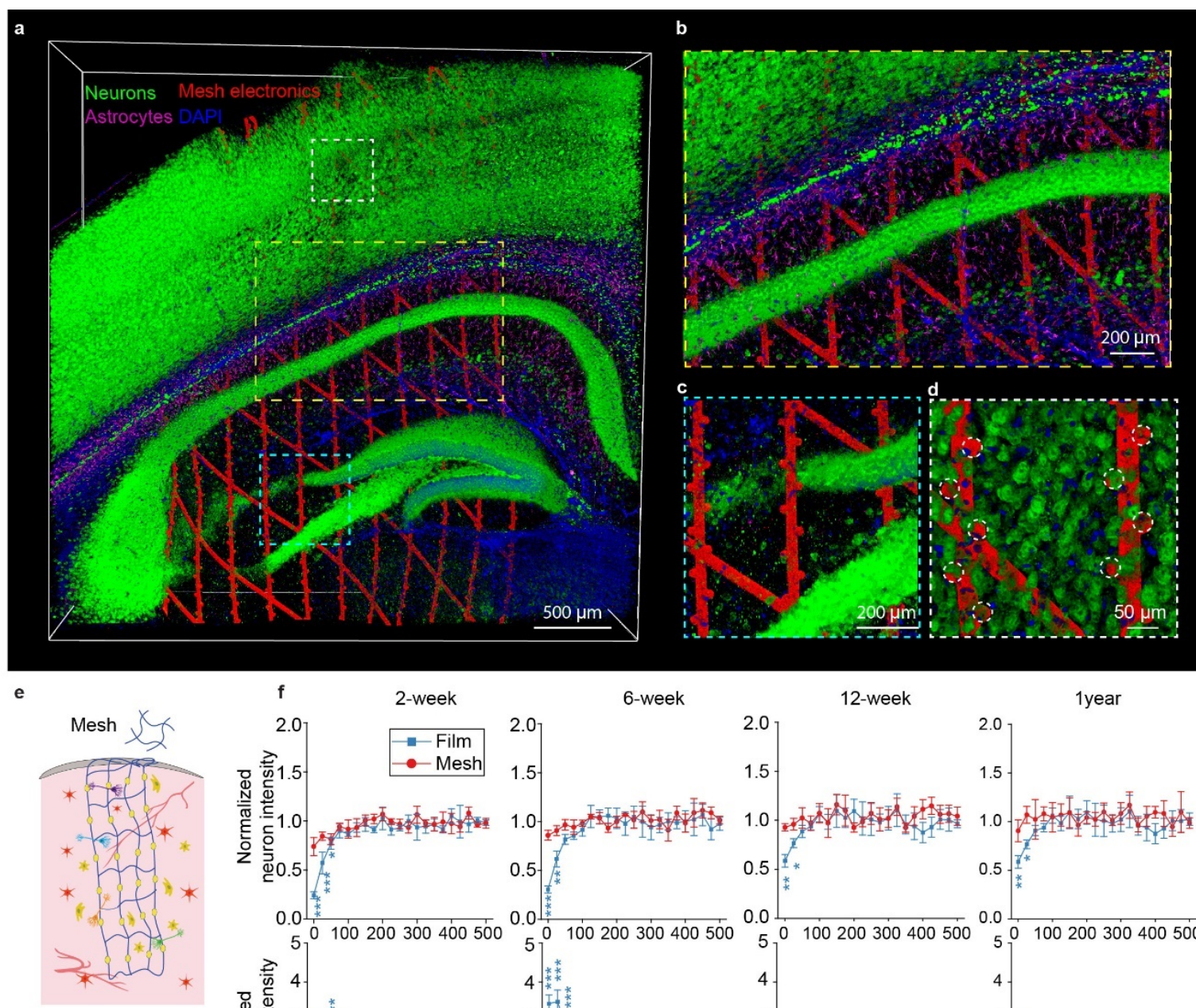

Thin film
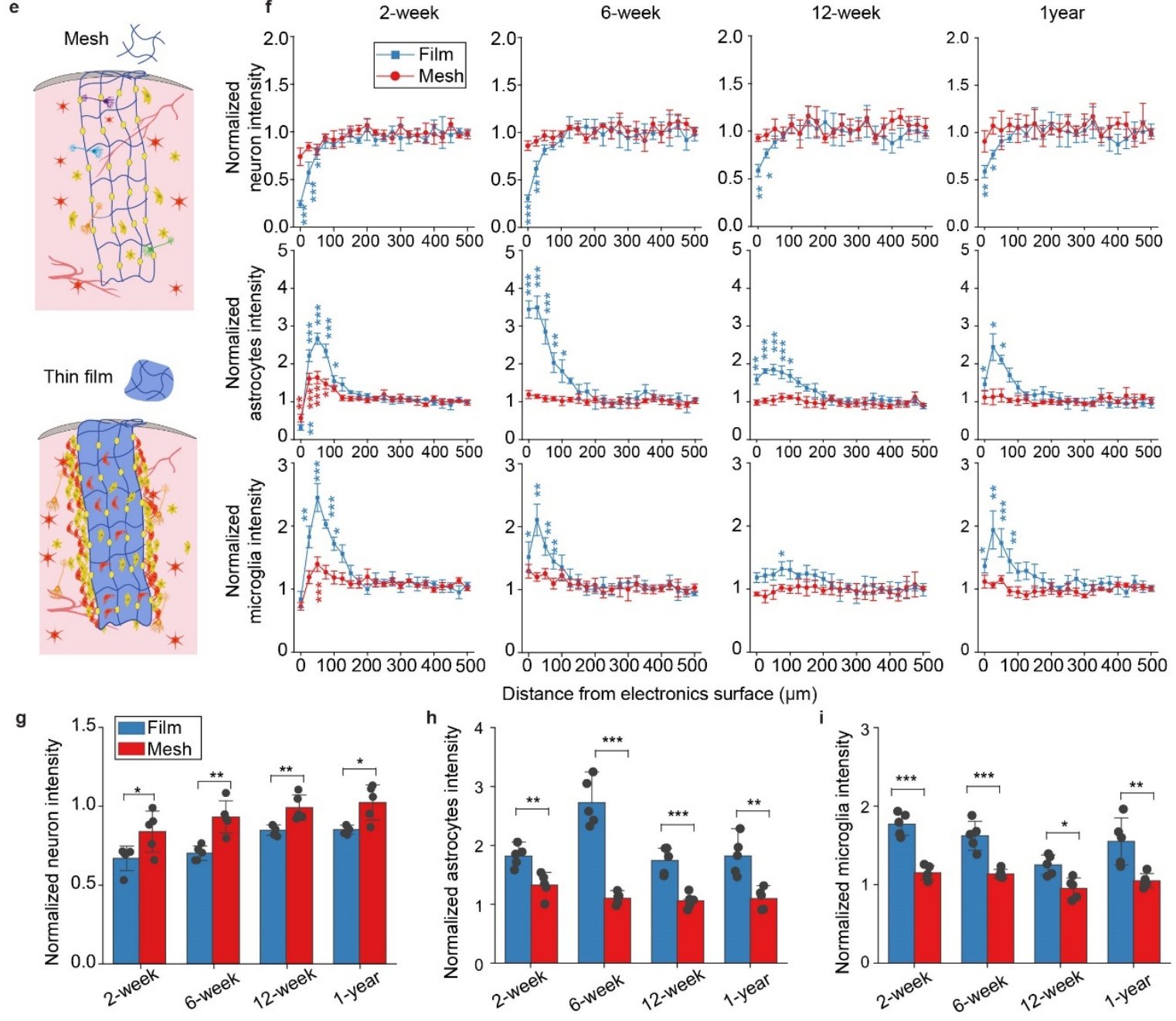
Fig. 2 | Unfolded mesh nanoelectronics seamlessly integrating with the neuron network across multiple brain regions. a, Representative $3 \mathrm{D}$ reconstructed confocal fluorescence imaging of $600-\mu \mathrm{m}$-thick brain tissue implanted with 2-mm wide mesh nanoelectronics for 6 weeks. Green, purple, blue, and red label neurons, astrocytes, nuclei, and mesh nanoelectronics. b-d, Zoom-in views of the regions highlighted by white (b), cyan (c), and yellow (d) dashed boxes in (a), showing the seamless integration of the mesh with the neural network. Neuron-like electrodes are highlighted by white dashed circles in (d). e, Schematics illustrating 1- $\mu$ m-thick mesh (top) and thin-film (bottom) nanoelectronics implanted and unfolded inside brain tissue for the long-term immune response characterization. f, Normalized fluorescence intensity as a function of distance from the mesh/thin-film electronic and tissue boundary at 2 weeks, 6 weeks, 12 weeks, and 1-year post-implantation. The relative signal was obtained by normalizing the fluorescence intensity with the baseline value defined as the fluorescence intensity over a range of 525-550 $\mu \mathrm{m}$ away from the electronics. Data represented as mean \pm SEM (intensity compared with that of distance at 500$\mu \mathrm{m},{ }^{*} p<0.05, * * p<0.01,{ }^{* * *} p<0.001$, two-tailed unpaired $t$ test, $n=5$ ). g-i, Normalized neuron (g), astrocytes (h), and microglia (i) intensity and neuron density within $100-\mu \mathrm{m}$ from the electronic surface. Data represented as mean $\pm \mathrm{SD}$, individual data points are overlaid $(* p<0.05$, $*_{*}^{*} p<0.01,{ }^{* * *} p<0.001$, two-tailed unpaired $t$ test, $n=5$ ).

457 
bioRxiv preprint doi: https://doi.org/10.1101/2021.10.29.466524; this version posted November 1, 2021. The copyright holder for this preprint (which was not certified by peer review) is the author/funder. All rights reserved. No reuse allowed without permission.

a
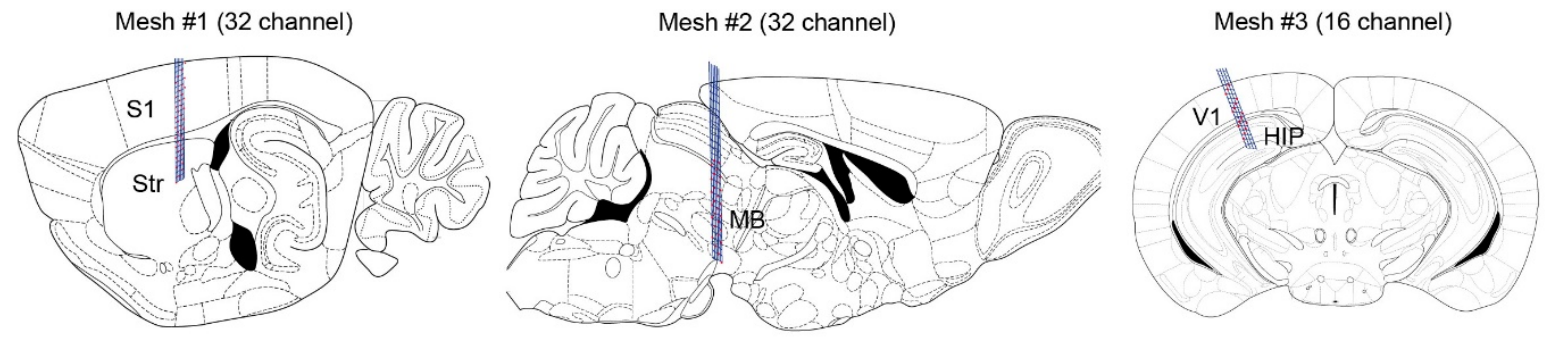

b

क
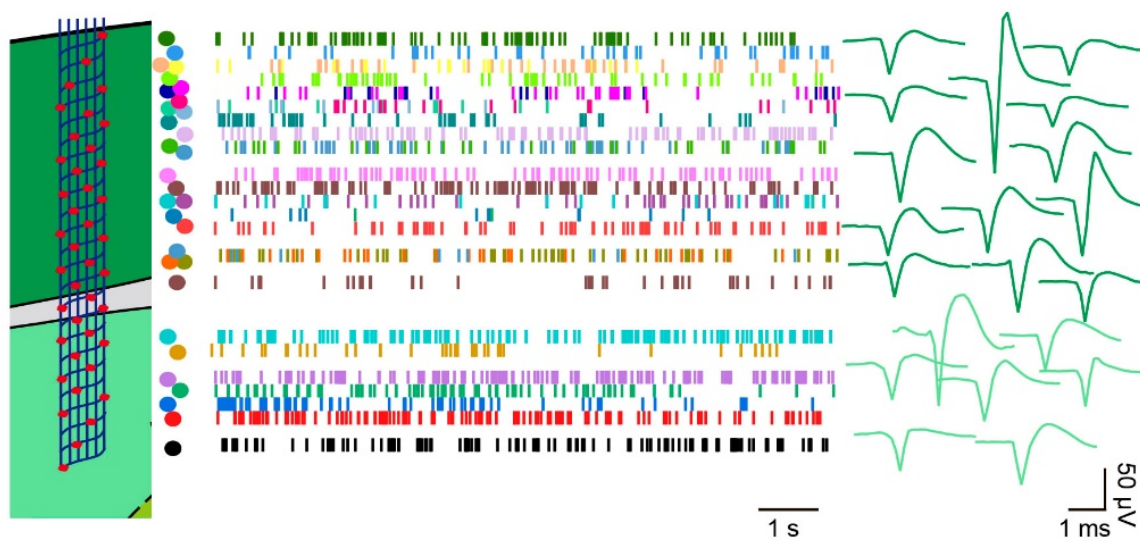

e

c

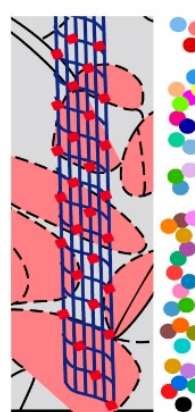

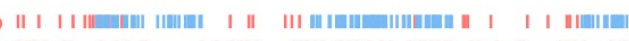
|III I| I II || II IIII IIIII IIIIIII I IIIII II I || IIII I| |

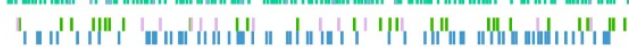

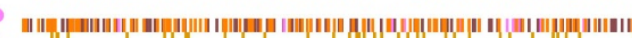

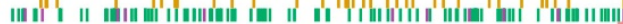

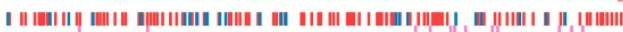

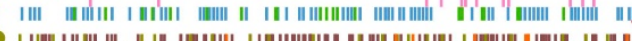

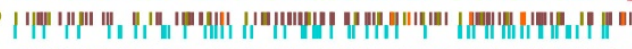

d

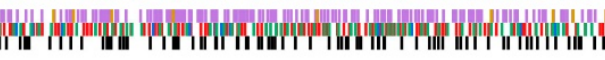
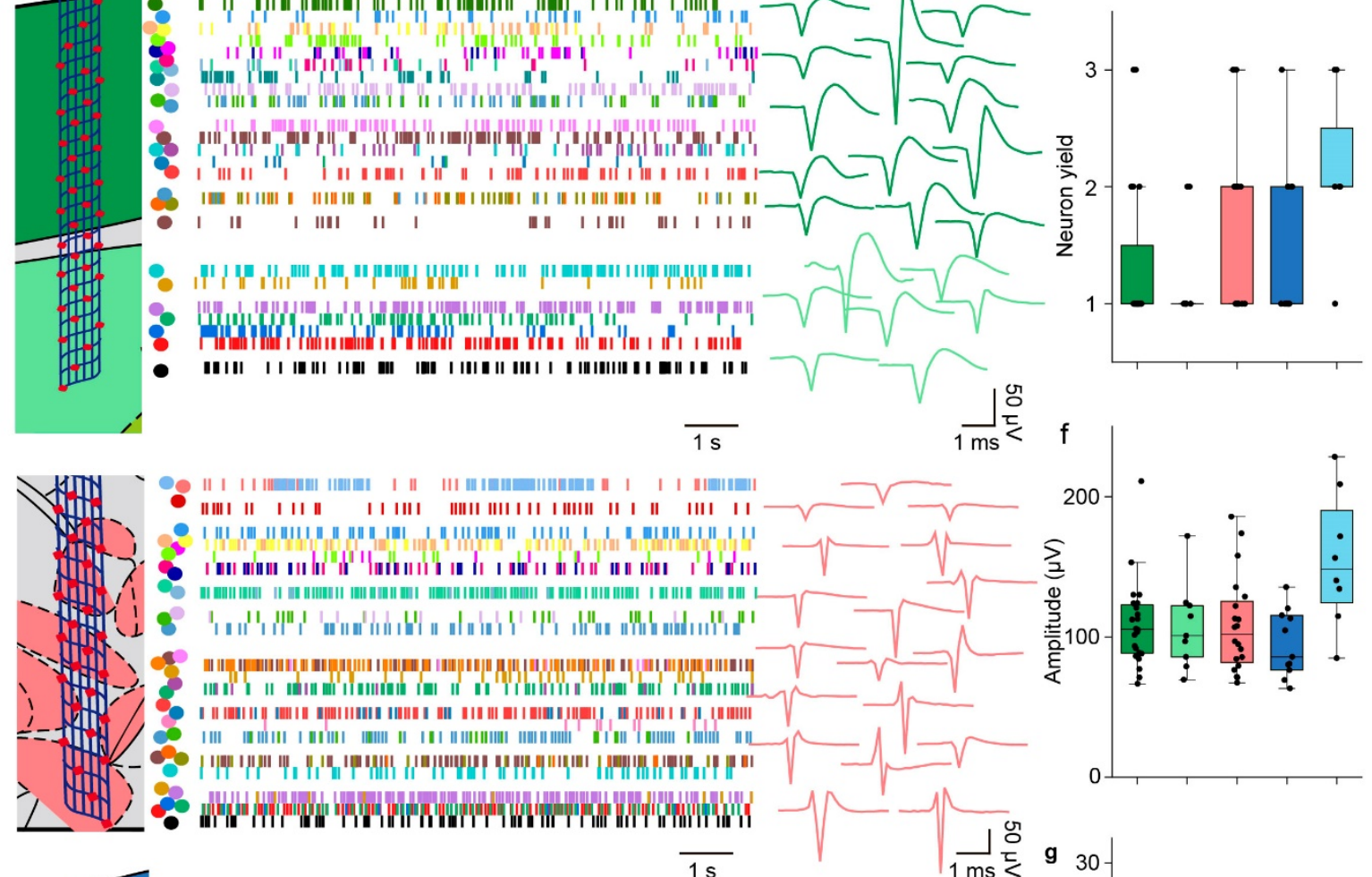

ร

| | ||||| || |||| |||| || | ||| || || || || | ||| ||| | ||||||||| || || || ||| ||| |||

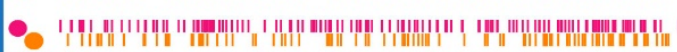

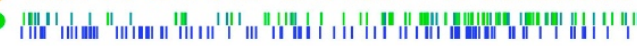

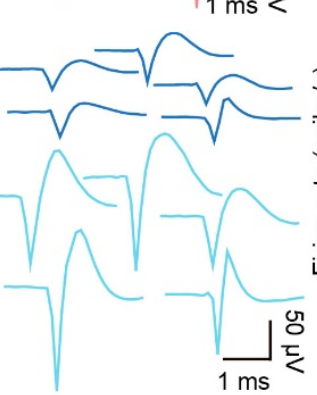

$\stackrel{\frac{0}{1}}{\bar{T}}$

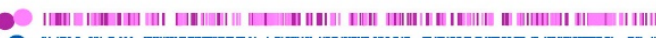

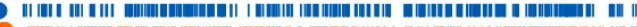
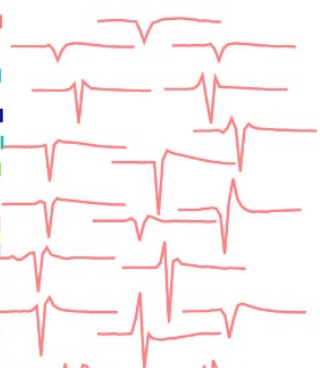

h

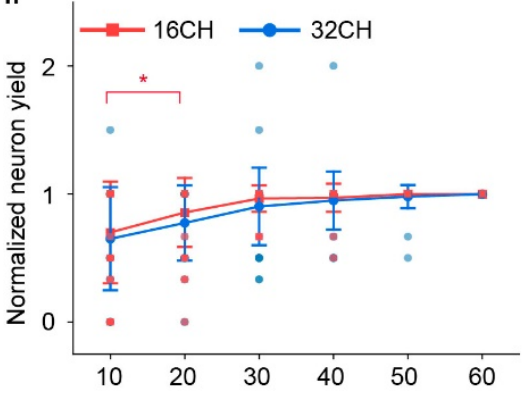

$\overline{1 s}$

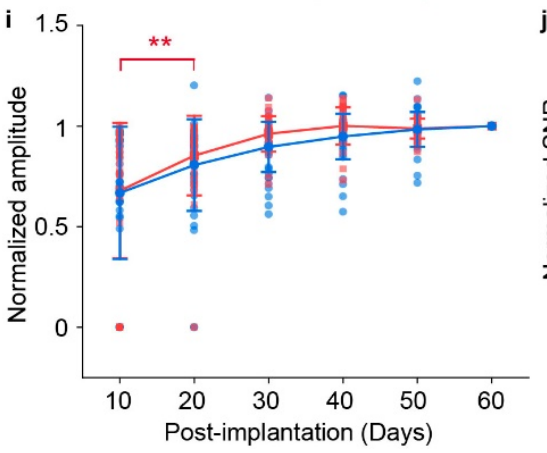

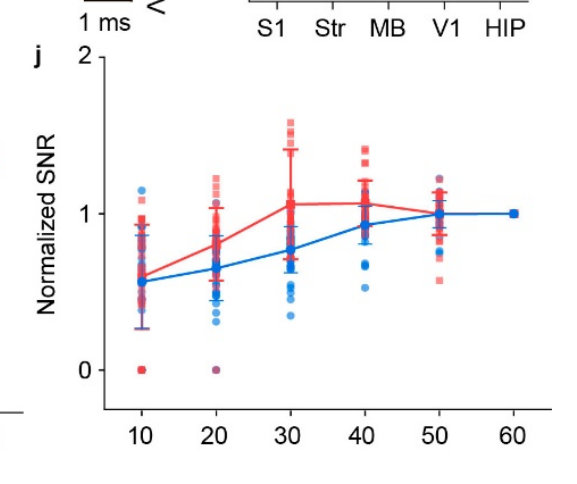


Fig. 3 | Chronically stable recording across multiple brain regions. a, Schematics illustrating the representative brain regions implanted with mesh nanoelectronics for chronic recording. S1, primary somatosensory cortex; Str, striatum; MB, midbrain; V1, primary visual cortex; HIP, hippocampus. b-d, Approximate mesh locations overlaid on the Allen Mouse Brain Atlas (left) and representative spiking raster (middle) and single-unit waveform (right) from two 32-channel $(\mathbf{b}, \mathbf{c})$ and one 16-channel (d) mesh nanoelectronics in head-fixed behaving animals at 60 days post-implantation. Colored dots and blocks indicate individual neurons and spike times, respectively (middle). Representative average single-unit action potential waveforms were extracted from a 2-min recording session (right). e-g, Quantification of sorted neuron yield per electrode (e), waveforms amplitude (f), and firing rate (g) across 5 brain regions in a 2 min recording session. Box plots show median and quartile range (whiskers denote $1.5 \times$ the interquartile range). Individual data points are overlaid ( $n=29$ electrodes from three 16-channel mesh nanoelectronics and $n=43$ electrodes from two 32-channel mesh). h-j, Normalized sorted neuron yield (h), amplitude (i), and signal-to-noise ratio (SNR) (j) over the time course of 60 days. Data represented mean $\pm \mathrm{SD}$, individual data points are overlaid $(n=29$ electrodes from three 16channel mesh nanoelectronics and $n=43$ electrodes from two 32-channel mesh nanoelectronics. ${ }^{*} p<0.05,{ }^{*} p<0.01$, comparison of different days within 16-,32-channel mesh electronics, twotailed unpaired $t$ test).

477 

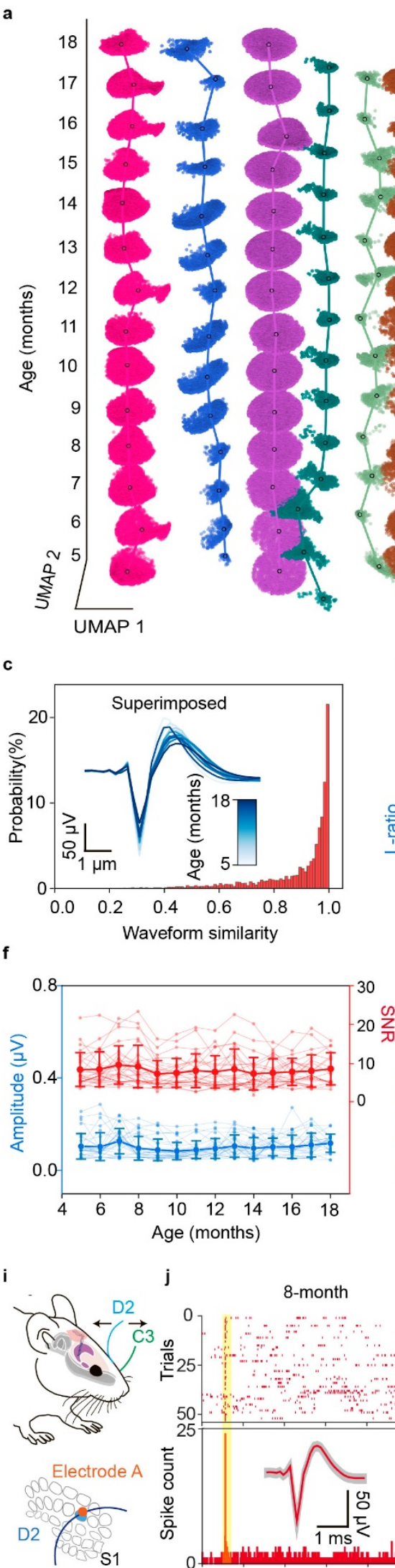

j
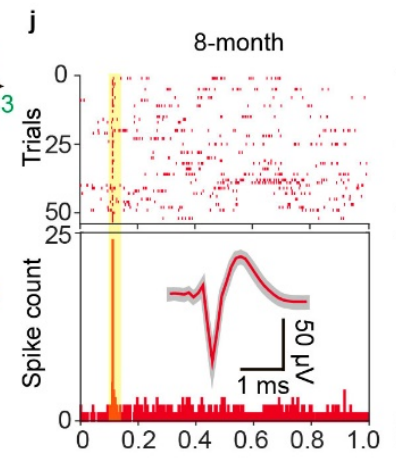
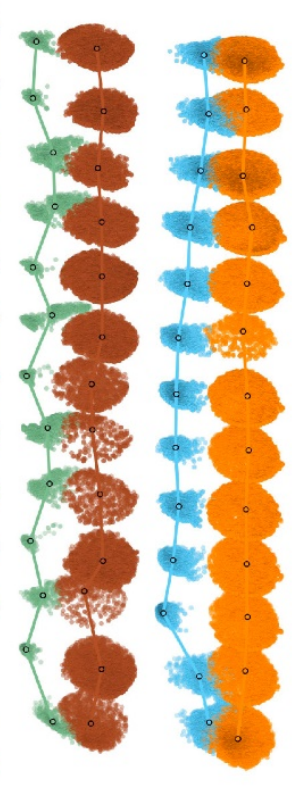

d

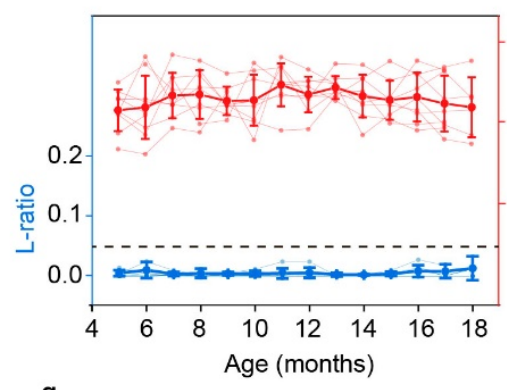

g

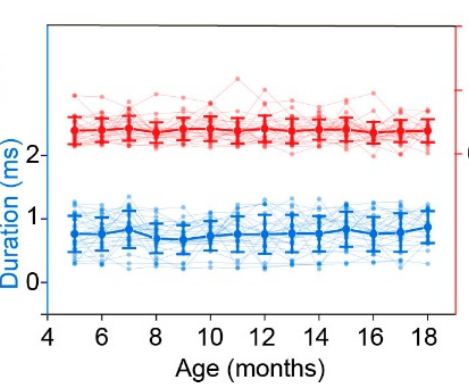

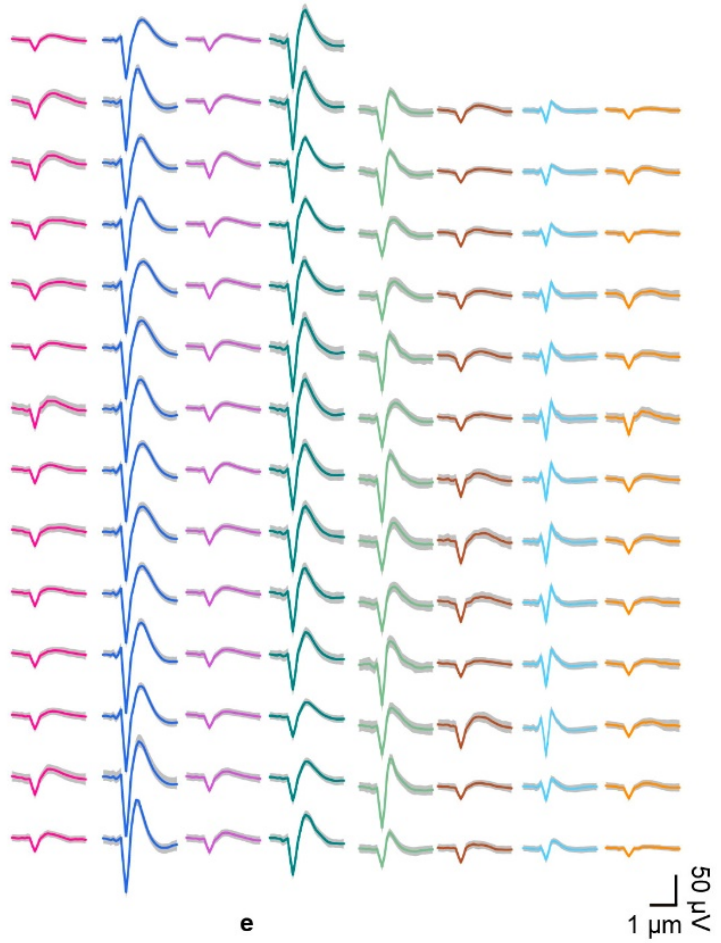

Repolarization slope Recovery slope

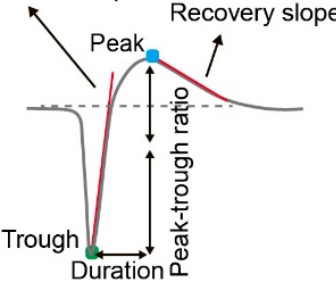

h

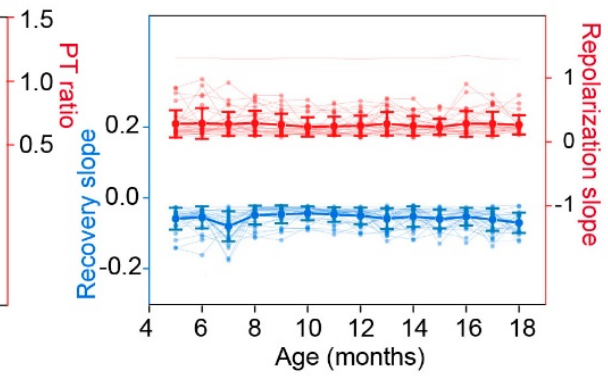

k

I
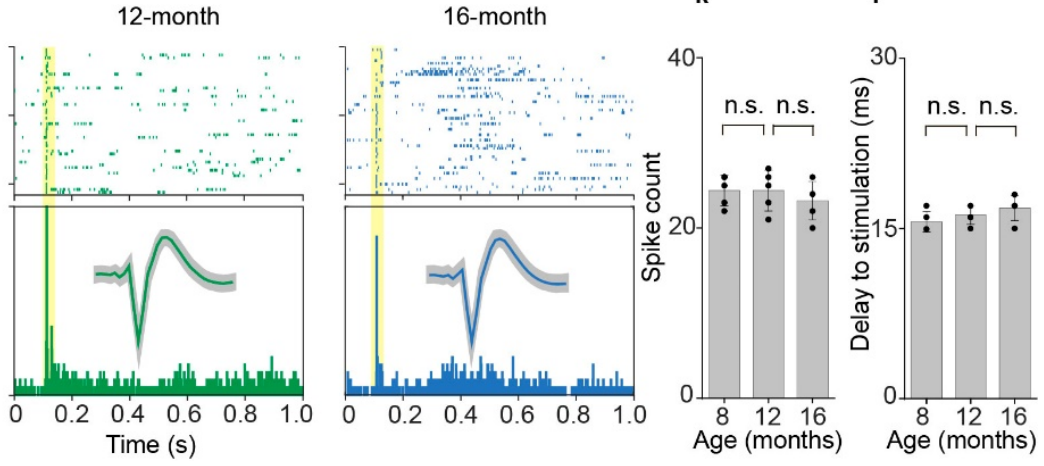
Fig. 4 | Stably tracking the single-unit action potential from the same group of neurons over the entire adult life of mice. a, Time evolution of single-units clustered by Leiden over the entire adult life of mice from the mature adult ( 5 months) to the aged (18 months) stage. The $\mathrm{x}-$ and $\mathrm{y}-$ axes denote the first and second uniform manifold approximation and projection (UMAP) dimensions, respectively, and the z-axis denotes mouse age in months. Color scheme maintained throughout. b, Time course analysis of the average waveforms of single-unit action potential in the Leiden clustering results in (a). Waveform represented mean \pm SD. c, Waveform correlations for every single-unit across all recording sessions ( $n=26$ units from 3 mice). Inset: Overlaid average waveforms across all recording session for the representative putative same neuron. d, Likelihood-ratio (L-ratio) and silhouette score showing the clustering quality for the single unit action potentials from identified neurons. Data represented mean $\pm \mathrm{SD}$, individual data points are overlaid. The constant dashed line is 0.05 L-ratio, commonly taken as a threshold of high cluster quality. e, Schematic showing features extracted from the single-unit action potential waveform used for the analysis in (f-h). $\mathbf{f}-\mathbf{h}$, mean \pm SD (individual data points are overlaid) of features illustrated in (e) and signal-to-noise ratio (SNR) over three mice life recording. In order, amplitude, SNR, duration, peak-trough ratio (PT ratio), recovery slope, and repolarization slope are shown. i1, Long-term stable recording of the behavior-associated neuron. i, Schematic diagram of whisker deflection. An individual vibrissa was deflected in the rostral-caudal plane using a computercontrolled galvanometer system. D2 and C3 whiskers are labeled in blue and green, respectively (top). Schematic diagram of whisker barrel arrangement in S1 (bottom). Evoked spike from electrode A was associated with D2 whisker deflection. Control experiments were shown in Extended Data Fig. 7. j, Raster plot and peri-stimulus time histogram (PSTH, $1 \mathrm{~ms}$ bin size) of the single unit identified from electrode A response to D2 whisker deflection from 8- to 16-month recording. Inset: average single-unit waveforms from recordings in the S1 in response to D2 whisker deflection over time, Waveform represented mean \pm SD. $\mathbf{k}$, Population data showing the number of spikes (out of 55 trials) evoked by whisker deflection over the time course of implantation. I, Time delay of the evoked spikes to the stimulation over the time course of

506 implantation (n.s.: not significant, two-tailed unpaired $t$ test, $n=5$ ). Individual data points are 507 overlaid. 
a

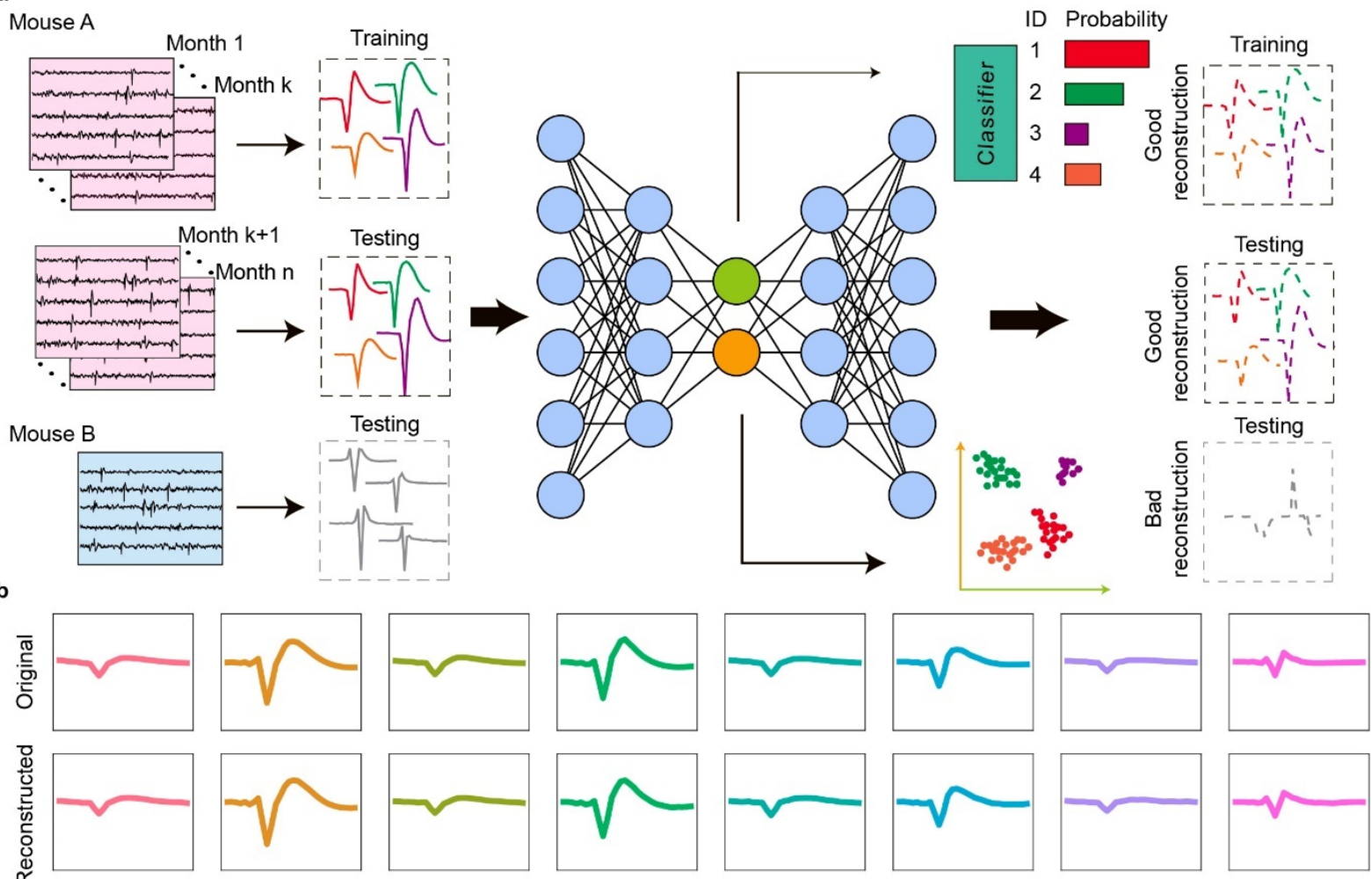

c

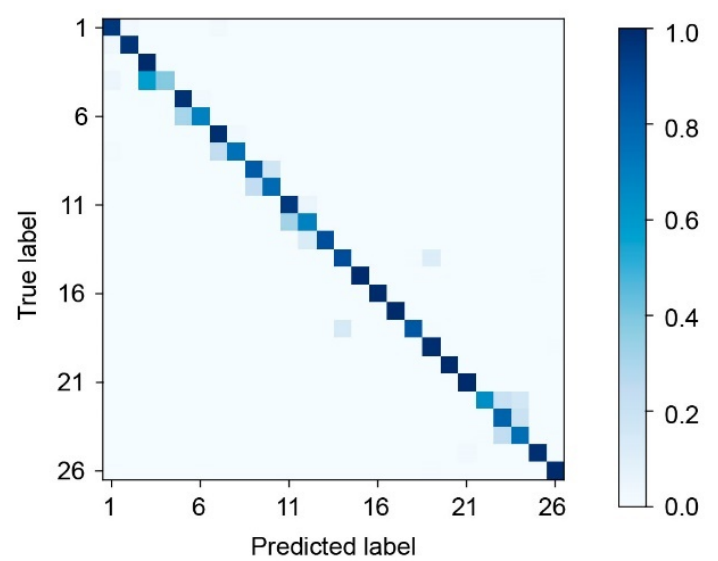

e

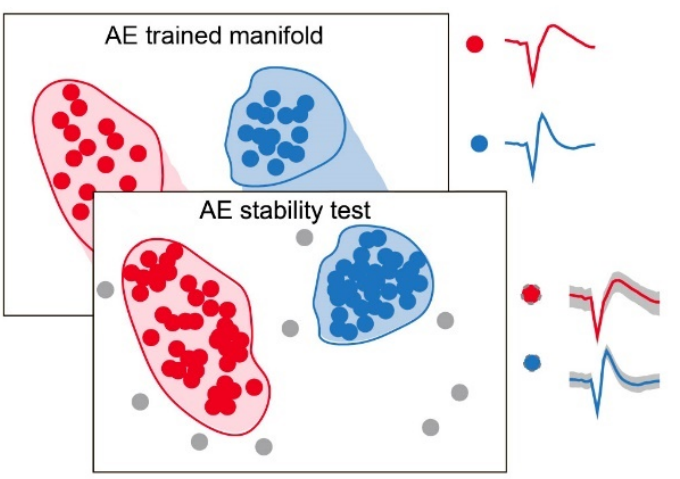

d
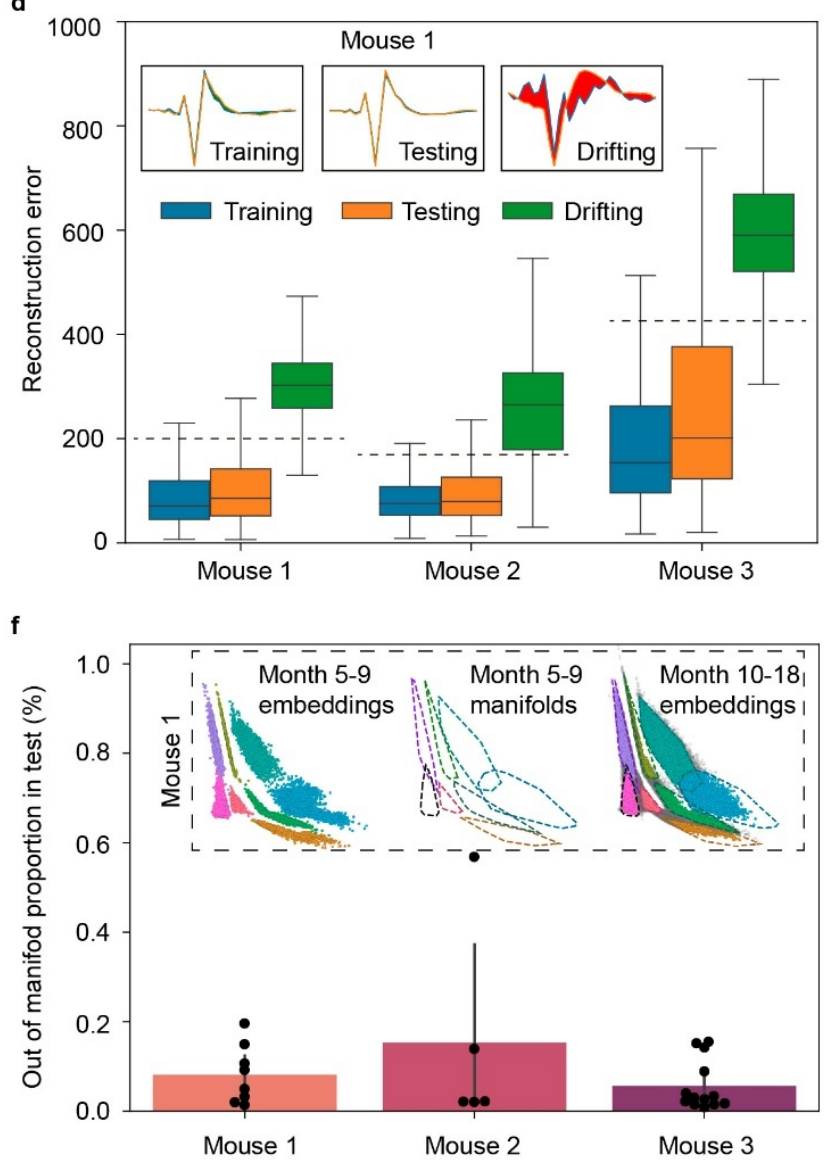
Fig. 5 | Long-term stable single-cell recording enabled autoencoder-based spike processing. a, Schematics of an autoencoder-based model which consists of an encoder, a classification head, and a decoder. Single-unit action potential waveforms are first extracted from the recording voltage traces and encoded with channel number to a lower-dimensional latent space. The latent representation is then used for classification and decoding. Spikes from the first 6 recording months including 5,490 spikes were used for training. The subsequent 8 months of data including 158,317 spikes were used for testing. Spikes from different groups of neurons collected from a different mouse were also used as simulated drifting data for testing. b, Original and reconstructed average representative single-unit waveforms from the testing data. Original waveforms are colored by neuron labels. Reconstructed waveforms are colored by the classification output. c, Confusion matrix comparing testing dataset ground-truth neuron labels with autoencoder classification output across all three mice. d, Boxplot of median and quartile range of meansquared error (MSE) distribution for each of the training, testing, and drift datasets. Whiskers denote $1.5 \times$ interquartile range. Dashed lines indicate thresholds corresponding to mean + SD of mouse-specific MSE training distribution, eliminating $83 \pm 4 \%$ of training and $86 \pm 7 \%$ of testing (Extended Data Fig. 8f). Inset: representative average waveforms and their corresponding reconstructions by the mouse-specifically trained autoencoder. The colored areas correspond to the difference between the reconstructed and original waveform. e, Schematics showing the autoencoder can be used to quantify the stability of the recording. The trained manifold (highlighted by red and blue) was used to test the stability of the recording by quantifying the percentage of the neuron single-unit action potentials from the testing data falling inside the training manifold. f, Bar plot illustrating the percentage of the testing dataset for each neuron falling outside of the training manifold as per the process illustrated in (e). Data represented mean through train-test waveform resemblance. Inset: applying noise rejection process to mouse 1 data, in order: training embeddings, training manifold boundaries, testing embeddings with out-ofmanifold spikes colored in grey. 
a

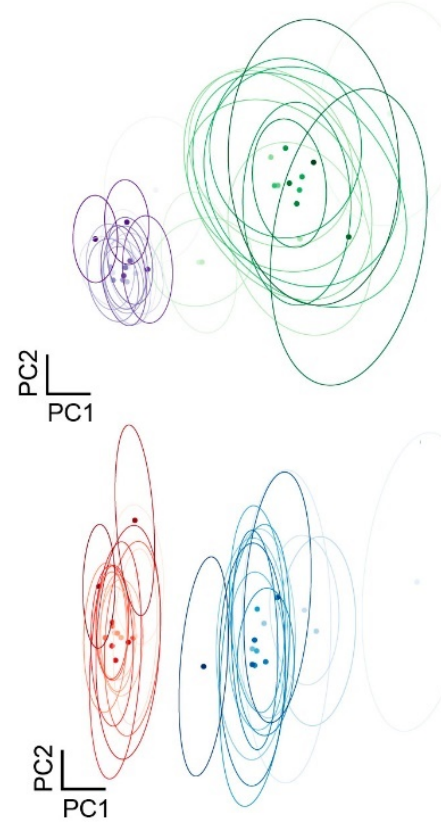

b

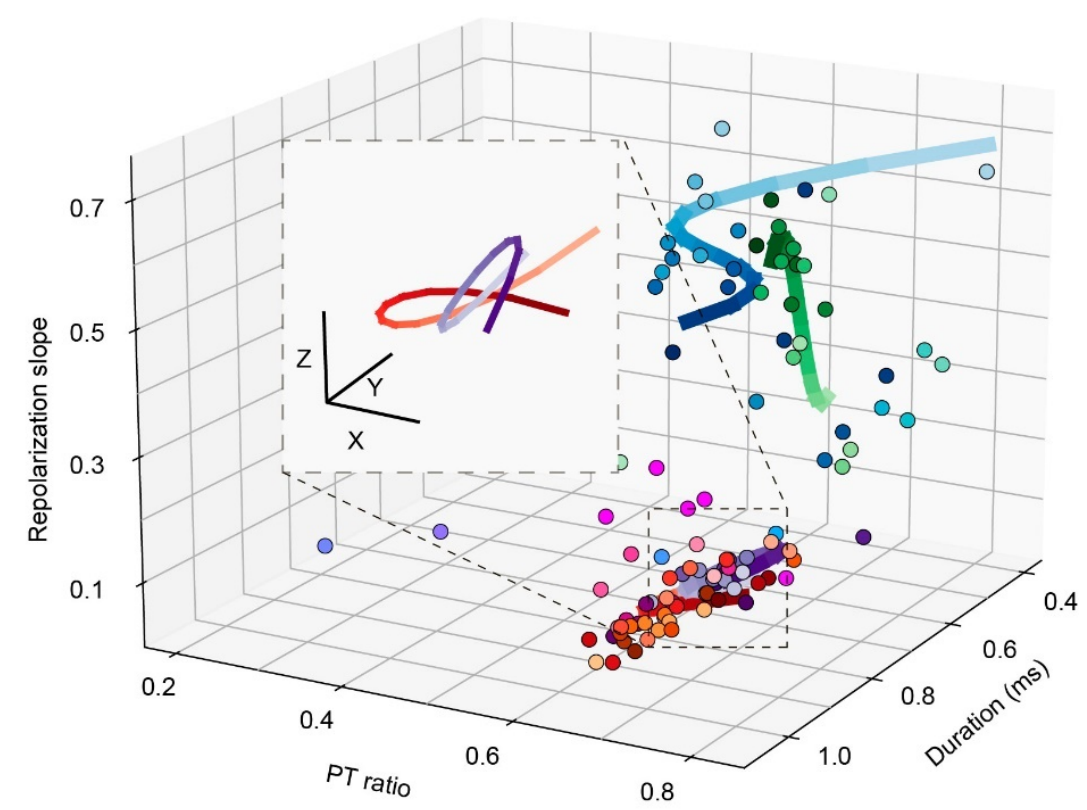

c

Real time

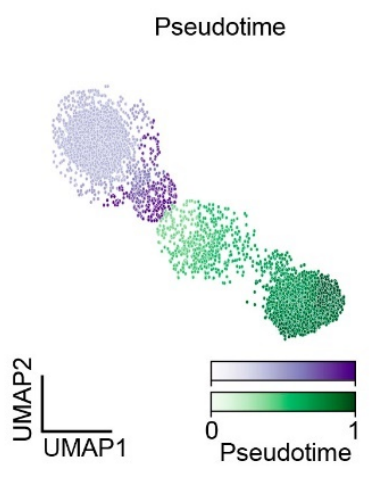

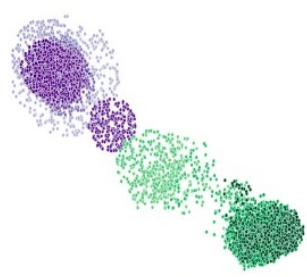

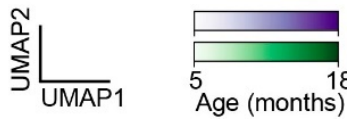

Fig. 6 | Adult life study of brain aging at the single-neuron level. a, Principal component analysis (PCA) of representative waveforms of neurons showing stable (purple and red) and progressive changing (green and blue) electrophysiological behaviors. Dots and ovals represent the centers and \pm 2 o of PC clusters, respectively. Average cluster centroid successive position shifts: 0.47 o $_{\text {purple, }} 0.58$ ored $^{\text {red }} 1.96$ ogreen, 2.63 oblue. Low values indicate stable cluster centroid position across time relative to single cluster whereas high values suggest time-evolution of corresponding cluster centroids and associated single-unit action potential waveforms. In both cases, a single electrode channel tracked stable $(<1 \%)$ and time-evolving single-unit action potential waveform. b. Trajectory analysis conducted with a B-spline interpolation of representative waveform features over the time course of implantation for the same neurons. Dots with the same color represented mean values of features calculated by the waveforms associated with the same color-coded neurons from data shown in (a). Trajectories highlight the representative neurons shown in (a) showing slow progressive and aging-associated electrophysiological properties. Inset: zoom-in view of purple and red trajectory. Scale bar, $\mathrm{x}=$ 
$5510.01, y=0.025, z=0.01$. c, Comparison of the electrophysiology Monocle3-based pseudotime and 552 real time evolution over the adult life of mice of overall UMAP representation of representative 553 individual single-unit action potential waveforms. Pairs of neurons recorded by the same electrode 554 are compared. 


\section{Methods}

\section{Fabrication of ultra-thin shuttle monolithically integrated mesh nanoelectronics}

(1) Cleaning a silicon wafer grown with thermal oxide (500-nm thickness) with acetone, isopropyl alcohol, and deionized water. (2) Depositing 100-nm-thick nickel (Ni) using a Sharon thermal evaporator as a sacrificial layer. (3) Spin-coating SU-8 precursor (SU-8 2000.5, MicroChem) at $3000 \mathrm{rpm}$, which was pre-baked at $\left(65^{\circ} \mathrm{C}, 95^{\circ} \mathrm{C}\right)$ for $2 \mathrm{~min}$ each, exposed to $365 \mathrm{~nm}$ ultra-violet (UV) for $200 \mathrm{~mJ} / \mathrm{cm}^{2}$, post-baked at $\left(65^{\circ} \mathrm{C}, 95^{\circ} \mathrm{C}\right)$ for $2 \mathrm{~min}$ each, developed using SU-8 developer (MicroChem) for $60 \mathrm{~s}$, and hard-baked at $180{ }^{\circ} \mathrm{C}$ for $40 \mathrm{~min}$ to define mesh SU-8 patterns for bottom encapsulation. (4) Spin-coating LOR3A photoresist (MicroChem) at $4000 \mathrm{rpm}$, followed by pre-baking at $180{ }^{\circ} \mathrm{C}$ for $5 \mathrm{~min}$; spin-coating S1805 photoresist (MicroChem) at 4000 $\mathrm{rpm}$, followed by pre-backing at $115^{\circ} \mathrm{C}$ for $1 \mathrm{~min}$; the sample was then exposed to $405 \mathrm{~nm} \mathrm{UV}$ for $40 \mathrm{~mJ} / \mathrm{cm}^{2}$, and developed using CD-26 developer (Microposit) for $70 \mathrm{~s}$ to define interconnects patterns. (5) Depositing 5/70/5-nm-thick chromium/gold/chromium ( $\mathrm{Cr} / \mathrm{Au} / \mathrm{Cr}$ ) by a Denton electron-beam evaporator, followed by a standard lift-off procedure in remover PG (MicroChem) to define the $\mathrm{Au}$ interconnects. (6) Repeating Step (4) to define electrode array patterns in LOR3A/S1805 bilayer photoresists. (7) Depositing 5/50-nm-thick chromium/platinum (Cr/Pt) by a Denton electron-beam evaporator, followed by a standard lift-off procedure in remover PG (MicroChem) to define the electrode array. (8) Repeating Step (3) for top SU-8 encapsulation. (9) Spin-coating SU-8 precursor (SU-8 2025, MicroChem) at $4000 \mathrm{rpm}$, which was pre-baked at $65^{\circ} \mathrm{C}$ for $2 \mathrm{~min}$ and $95^{\circ} \mathrm{C}$ for $8 \mathrm{~min}$, exposed to $365 \mathrm{~nm}$ ultra-violet (UV) for $200 \mathrm{~mJ} / \mathrm{cm}^{2}$, post-baked at $65^{\circ} \mathrm{C}$ for $2 \mathrm{~min}$ and $95^{\circ} \mathrm{C}$ for $6 \mathrm{~min}$, developed using SU-8 developer (MicroChem) for $6 \mathrm{~min}$, and hard-baked at $180^{\circ} \mathrm{C}$ for 1 hour to define SU-8 anchors patterns to connect the mesh and SU8 shuttle. (10) Spin-coating $20 \mathrm{wt} \%$ dextran solution at $1000 \mathrm{rpm}$ for $20 \mathrm{~s}$. which was at $80{ }^{\circ} \mathrm{C}$ for 

width of SU-8 scaffolds, $w_{m}$ is the width of gold interconnects, $h_{m e s h}=0.91 \mu \mathrm{m}$ and $h_{\text {film }}=0.92 \mu \mathrm{m}$

$1 \mathrm{~min}$ and $180{ }^{\circ} \mathrm{C}$ for $30 \mathrm{~min}$. (11) Spin-coating SU-8 precursor (SU-8 2025, MicroChem) at 3000 $\mathrm{rpm}$, which was pre-baked at $65^{\circ} \mathrm{C}$ for $2 \mathrm{~min}$ and $95^{\circ} \mathrm{C}$ for $8 \mathrm{~min}$, exposed to $365 \mathrm{~nm}$ ultra-violet (UV) for $200 \mathrm{~mJ} / \mathrm{cm}^{2}$, post-baked at $65{ }^{\circ} \mathrm{C}$ for $2 \mathrm{~min}$ and $95{ }^{\circ} \mathrm{C}$ for $6 \mathrm{~min}$, developed using SU-8 developer (MicroChem) for $6 \mathrm{~min}$, and hard-baked at $180{ }^{\circ} \mathrm{C}$ for 1 hour to define the SU-8 shuttle pattern. (12) Cleaning the input/output with water and soldering a 32-channel flexible flat cable (Molex) onto the input/output pads using a flip-chip bonder (Finetech Fineplacer). (13) Soaking the mesh nanoelectronics in nickel etchant for 2 to 4 hours to completely release the mesh nanoelectronics from the substrate wafer. (14) Rinsing the mesh nanoelectronics with deionized water and PBS three times each. (15) Dicing the substrate to the desired length. Dip-coating 10 wt $\%$ PEG solution to attach the mesh nanoelectronics and SU-8 polymer shuttle. The monolithically integrated mesh nanoelectronics was allowed to dry in the air. (16) After cutting the anchor, the monolithically integrated mesh nanoelectronics was ready for implantation.

\section{Bending stiffness calculations}

We estimated and compared the bending stiffness values (D) of mesh and thin-film nanoelectronics using a beam model. The bending stiffness of mesh nanoelectronics with three-layer polymer/mesh/polymer structure can be calculated as

$$
\mathrm{D}=\frac{E_{S}}{12}\left(w h^{3}-w_{m} h_{m}^{3}\right)+\frac{E_{m}}{12} w_{m} h_{m}^{3}
$$

where $E_{\mathrm{s}}$ and $\mathrm{E}_{\mathrm{m}}$ are the young's moduli of SU8 and gold, 2 and $79 \mathrm{GPa}$, respectively, $w$ is the is the measured total thickness of SU-8, $h_{\text {metal }}=80 \mathrm{~nm}$ is the thickness of Cr/Au interconnects. The 
599 calculated bending stiffness of mesh and thin-film nanoelectronics, $1.26 \times 10^{-15} \mathrm{~N} \cdot \mathrm{m}^{2}$ and $39.8 \times 10^{-}$

$600 \quad{ }^{15} \mathrm{~N} \cdot \mathrm{m}^{2}$, respectively.

601

602

\section{Brain implantation}

603 All the animal experiments were approved by the Institutional Animal Care and Use Committee 604 of Harvard University. The implantations were carried out on the male C57BJ/6 mice $(\sim 16$ weeks 605 of age). The animals were housed in a regular $12 \mathrm{~h} / 12 \mathrm{~h}$ light/dark cycle. Animals were 606 anesthetized with 2-3\% isoflurane and maintained under anesthesia with $0.75-1 \%$ isoflurane 607 during the intracranial implantation surgery. Stainless-steel screws were implanted in the 608 cerebellum and used as ground electrodes. A craniotomy $\left(2 \times 2 \mathrm{~mm}^{2}\right)$ was performed on the brain, 609 and the cortical surface was exposed upon removal of the dura mater. The mesh nanoelectronics 610 with the releasable shuttle was attached onto a micromanipulator on the stereotaxic frame. The 611 micromanipulator was manually controlled to insert the nanoelectronics into the mouse brain at 612 the targeted depth at the tip. Sterile PBS was applied on the rear end of the nanoelectronics to 613 dissolve the PEG/dextran and release the SU-8 shuttle from mesh nanoelectronics. After 614 PEG/dextran was fully dissolved on both ends, the SU-8 shuttle was extracted with the second 615 manipulator, leaving ultra-flexible mesh nanoelectronics implanted at the target positions.

616 Craniotomies were sealed with a silicone elastomer (World Precision Instruments, USA). Ceramic 617 bone anchor screws, together with dental methacrylate, were used to fix the FFC and electrode set 618 onto the mice's skull.

\section{Immunohistochemistry}


621 The following procedures were performed according to our previous reports ${ }^{18,48}$

622

623

624

625

626

627

628

629

630

631

632

633

634

635

636

637

638

639

640

641

642

643

Histology sample preparation: At each time point (2-, 6-, 12-week and 1-year post-implantation), mice were anesthetized with $40-50 \mathrm{mg} / \mathrm{kg}$ sodium pentobarbital and then transcardially perfused with $\sim 40 \mathrm{ml}$ PBS pre-wash, and $\sim 40 \mathrm{ml} 4 \%$ paraformaldehyde (PFA) in PBS, followed by decapitation. The scalp skin was removed, and the exposed skull/dental cement were ground for 10-20 min at 15,000 r.p.m. using a high-speed micro motor tool. The brain with the mesh/thin film nanoelectronics undisturbed was removed from the cranium and postfixed in PFA for $24 \mathrm{~h}$ at $4{ }^{\circ} \mathrm{C}$. The brain was transferred to incrementally increasing sucrose solutions (10-30\%, w/v) until sunk to the bottom for the thin tissue preparation.

Immunohistochemical staining of $20-\mu \mathrm{m}$ thin tissue: After cryostat sectioning, brain slices were incubated PBST $(1 \times$ PBS with $0.2 \%$ Triton X-100, Thermo Fisher Scientific) for 30 min, and then blocked with $5 \%(\mathrm{w} / \mathrm{v})$ normal donkey serum for 2 hours. After three rinses with PBST for 30 min each, slices were then incubated at $4{ }^{\circ} \mathrm{C}$ overnight in the primary antibodies: chicken anti-glial fibrillary protein GFAP (targeting astrocytes, 1:200, Abcam \#ab4674, USA), goat anti-ionized calcium binding adaptor molecule 1 (Iba1) (targeting microglia, 1:100, Abcam \#ab5076, USA), and rabbit anti-neuronal nuclear NeuN (targeting nuclei of neurons, 1:200, Abcam \#ab177487, USA), followed by the slices being washed three times for $30 \mathrm{~min}$ each with PBST. Slices were then incubated in a secondary antibody solution at room temperature for $2 \mathrm{~h}$ with protection from light (1:500, Alexa Fluor 647 donkey anti-chicken, Jackson Immunoresearch, USA; 1:500, Alexa Fluor 594 donkey anti-goat; 1:500, Alexa Fluor 488 donkey anti-rabbit, Invitrogen, USA). After being washed three times for 30 min each with PBST, brain slices were also stained by incubating with 4',6-diamidino-2-phenylindole (DAPI, Sigma-Aldrich, USA) to mark all cell nuclei for 30 min. After being washed, slices were mounted on glass slides with coverslips using Prolong Gold 
644 (Invitrogen, USA) mounting media. The slides remained covered (protected from light) at room temperature, allowing for $12 \mathrm{~h}$ of clearance before imaging.

646 Tissue clearing and staining for thick tissues: After vibratome sectioning, brain slices were 647 placed in $1 \times$ PBS containing 4\% (w/v) acrylamide (Sigma-Aldrich) and 0.25\% (w/v) VA-044 648 thermal polymerization initiator (Fisher Scientific) at $4{ }^{\circ} \mathrm{C}$ for 3 days. The solution was replaced 649 with fresh solution immediately before placing the brain slices in X-CLARITY polymerization system (Logos Biosystems) for $3 \mathrm{~h}$ at $37^{\circ} \mathrm{C}$. After polymerization, any remaining gel from the tissue surface was removed and the slices were rinsed with PBST before placing them in electrophoretic tissue clearing solution (Logos Biosystems) at $37^{\circ} \mathrm{C}$ for $3-5$ days until the samples were translucent. Brain slices were incubated with PBST overnight, followed by three washes with PBST, and then blocked with $5 \%(\mathrm{w} / \mathrm{v})$ normal donkey serum for 2 days. After three rinses with PBST, slices were then incubated at $4^{\circ} \mathrm{C}$ for 5-7 days in the primary antibodies containing: chicken anti-glial fibrillary acidic protein (GFAP) (targeting astrocytes, 1:200, Abcam \#ab4674, USA) and/or and NeuN (targeting nuclei of neurons, 1:200, Abcam \#ab177487, USA), followed by the

658 slices being washed three times with PBST. Slices were then incubated in a secondary antibody solution at $4{ }^{\circ} \mathrm{C}$ for 5-7 days with protection from light (1:500, Alexa Fluor 647 donkey anti-

660 chicken, Jackson Immunoresearch, USA; 1:500 and/or Alexa Fluor 488 donkey anti-rabbit,

\section{Microscope imaging and image data analysis}


667 Confocal fluorescent images were acquired using a Leica SP8 confocal system (Leica, USA).

668 Images were collected using a $25 \times, 0.95 \mathrm{NA}$ water-immersion or $40 \times, 1.3 \mathrm{NA}$ oil-immersion

669 objective lens. $488 \mathrm{~nm}, 591 \mathrm{~nm}$, and $633 \mathrm{~nm}$ lasers as the excitation sources for Alexa Fluor 488,

670 Alexa Fluor 594, and Alexa Fluor 647, respectively. Standard TIFF files were exported and

671 colorized using LAS X Software. ImageJ software and custom code were used for image analysis.

672 The distance of each pixel to mesh/film nanoelectronics was defined as its shortest distance from

673 the mesh/film boundary. Baseline fluorescence intensity is defined as the average fluorescence

674 intensity of all pixels 525-550 $\mu \mathrm{m}$ away from the boundary. Intensity values with distances binned

675 over an interval of $25 \mu \mathrm{m}$ were averaged and normalized against the baseline intensity.

676

\section{6. Electrophysical measurement}

678 Mice with implanted mesh nanoelectronics and FFC connector were recorded chronically monthly

679 using CerePlex Direct recording system (Blackrock Microsystem, USA), starting from 1-month 680 post-implantation. Mice were anesthetized with $1 \%$ isoflurane in medical-grade $\mathrm{O}_{2}$ or head-fixed

681 during the measurement. Homemade PCB for connecting the FFC and head stage. Two animals

682 are excluded from the long-term study (Fig. 4) since the poor body condition and connectors failed

683 at an early stage. The electrophysiological recording was made with a $30-\mathrm{kHz}$ sampling rate and a

$68460-\mathrm{Hz}$ notch filter. Whiskers of interest were trimmed at $\sim 15 \mathrm{~mm}$ from the face and inserted into

685 a polyimide tube fixed to the galvanometer system (PT-30K, SpaceLas, China) positioned $\sim 10 \mathrm{~mm}$

686 from the vibrissal pad to yield high-fidelity sensory stimuli. Stimulation was always delivered

687 along the rostro-caudal axis. Voltage command and output for the actuator were programmed by

688 Axon Digidata 1550B (Molecular Devices, USA). 


\section{Data analysis}

690

691

Spike sorting and clustering: All recording data was analyzed offline. 6 min continuous recordings were used for analysis of each month. Spike detection was performed using the WaveClus3 software package (https://github.com/csn-le/wave_clus). In brief, raw recording data was filtered using four poles Butterworth filters in the $300-3000 \mathrm{~Hz}$ frequency range before spikes were detected using an amplitude threshold 5 times the estimated standard deviation of the noise. After spike alignment, 30 data points with the sampling rate of $10 \mathrm{kHz}$ were kept for each detected spike representing $3 \mathrm{~ms}$. No normalization procedure was applied to the spike waveforms due to the stable nature of recorded amplitudes (Fig. 4f) and experiments (Extended Data Fig. 10a-f) showing normalization decreased putative neuron cluster separability as well as meaningfulness of PCA embeddings. Spike sorting results of WaveClus3 obtained through superparamagnetic clustering were kept and used for comparison with the results of our chosen spike sorting approach. Additional quality metrics calculated for individual neurons demonstrate that the individual neurons were clearly defined (Extended Data Fig. 10g-i). Results reported in Figure 4 and 6 used clusters determined by Leiden ${ }^{49}$ https://github.com/vtraag/leidenalg) clustering performed on the graph constructed by Uniform Manifold Approximation and Projection (UMAP, https://github.com/lmcinnes/umap) for each individual month and over individual channels. Geometric considerations ensured that no neuron was being recorded by two separate channels. Cluster labels were then aligned by choosing a label matching scheme which minimized the meansquared error between the average cluster waveforms of a given month and a chosen template.

Stability analysis of single-unit action potentials: After spike detection, sorting and clustering, stability analysis was performed. A custom computational pipeline for assessing the stability analysis, which was built using Python v3.9.4. Performing prior spike extraction using third-party 
712 software or using the pipeline to perform spike extraction and sorting directly in python are both

713 possible.

714 UMAP analysis was used to confirm the recording stability. UMAP embeddings' coordinates were 715 calculated for all spikes of a given channel before plotting the corresponding points per recording 716 month producing a visualization over time. The representations originating from different channels 717 were plotted next to each other after manual curation for quality control to ensure only the best 718 quality spikes were included in the analysis. Additionally (Fig. 6a), average cluster centroid 719 position shifts were compared to average cluster distributions spread. Concretely, let us name $X \in$ $720 \Re^{d x 2}$ a vector of cluster centroid positions over d days in a given $2 \mathrm{D}$ (x, y) embedding. For each 721 identified neuron, we calculated:

$$
\sqrt{\left(\frac{u_{x}}{\sigma^{a v_{x}}}\right)^{2}+\left(\frac{u_{y}}{\sigma^{a v_{y}}}\right)^{2}}
$$

723 with $u_{x}=\frac{1}{d-1} \sum_{i=i}^{d-1}\left|x_{i+1}-x_{i}\right|, u_{y}=\frac{1}{d-1} \sum_{i=i}^{d-1}\left|y_{i+1}-y_{i}\right|$ the average successive cluster 724 centroid absolute position shifts along each axis, and $\sigma^{a v}{ }_{x}=\frac{1}{d} \sum_{i=1}^{d} \sigma_{i}^{x}, \sigma^{a v}{ }_{y}=$ $725 \frac{1}{d} \sum_{i=1}^{d} \sigma^{y}{ }_{i}$ the average cluster distribution SD along each axis. This allowed us to compare 726 centroid position shifts with average cluster SD along each axis to examine the embedded 727 waveform stability. Results of correlation analysis using Pearson correlation coefficients and 728 associated two-sided $t$ tests were carried out using scipy v1.6.3 (http://www.scipy.org) stats 729 module.

730 Cluster quality metrics shown in Figure $4 d$ were obtained by calculating silhouette score using 731 scikit-learn v0.24.2's (http://scikit-learn.org) implementation over individual channels and months. 


$$
L_{\text {ratio }}(C)=\frac{L(C)}{n_{c}}
$$

$$
\text { where, } L(C)=\sum_{i \notin C} 1-C D F_{\chi_{d f}^{2}}\left(D_{i, C}{ }^{2}\right)
$$

735

736

737

with $n_{c}$ the number of spikes belonging to cluster $\mathrm{C}, C D F_{\chi^{2}}{ }_{d f}$ the cumulative distribution function of the $\chi^{2}$ distribution, $d f=2$ in our case and $D_{i, C}{ }^{2}$ being the mahalanobis distance of spike i from the center of cluster C. Linear regressions were performed using scikit-learn. Within unit correlation analysis in Figure. $4 \mathrm{c}$ was calculated based on the Pearson product-moment correlation coefficient. The corrcoef function in numpy v1.18.5 (http://www.numpy.org) was used between all pairs of average cluster waveforms and recording days to provide the auto and cross correlation coefficients. Feature extraction was performed by using functions adapted from AllenSDK (https://github.com/AllenInstitute/AllenSDK).

Analysis of interspike intervals: The spiking times of each sorted neuron were used to calculate the interspike interval (ISI) histograms for individual months per cluster with a bin size of $2 \mathrm{~ms}$.

Data analysis of whisker-stimulus provoked recording: For whisker responsive recording, firing timing of each detected single-unit spike was presented and aligned by trial number in the raster plot. For the peristimulus time histogram (PSTH), $1 \mathrm{~ms}$ bin size was used and the spike counts were accumulated from all trials within each recording session.

Autoencoder-based automatic spike processing: All autoencoders were built using tensorflow 2.5 (https://www.tensorflow.org). The encoder consisted of two fully connected (FC) layers with 100 nodes each before the bottleneck 2D layer. Inputs to the network were concatenations of individual spike waveforms and their corresponding one-hot encoded channel (geometric considerations of the sparsity of the mesh recording device ensure that no single neuron can be 
754

755

756

757

758

759

760

761

762

763

764

765

766

767

768

769

770

771

772

773

774

775

776

recorded on two separate channels). From the bottleneck, the classification head, which consists of a single FC layer with softmax activation, outputs probabilities of belonging to a particular neuron class. The decoder's symmetric structure with respect to the encoder allows for reconstruction of the original waveform. Activations for encoder and decoder layer were set as LeakyReLU with alpha $=0.3$ as well as an added $L_{2}$ regularizer on the encoder's last layer, enforcing lower latent embedding values. Adam optimizer with default parameters was used. For a given dataset, $X \in R^{n \times 30}, n \in$ Nof $\mathrm{n}$ spikes, each composed of 30 data points recorded on $\mathrm{c}$ channels, we denote the input batch to the autoencoder $\hat{X} \in R^{p x(30+c)}$. The autoencoder considered here is composed of an encoder, $f_{e}$, decoder $f_{d}$ and classifying head $f_{c}$ parametrized by $\theta=\left(\theta_{e}, \theta_{d}, \theta_{c}\right)$. The network's (slightly simplified, as the encoder's last layer regularization is not explicit here) loss function over a batch of $\mathrm{p}$ spikes is thus:

$$
L(\widehat{X}, \theta)=\sum_{i=1}^{p}\left[\alpha|| x_{i}-f_{d}\left(f_{e}\left(x_{i}\right)\right)||_{2}^{2}+\beta C E\left(f_{c}\left(f_{e}\left(x_{i}\right)\right)\right)\right]
$$

where CE is the usual categorical cross-entropy loss used for multi-class classification problems.

The loss function is clearly defined by two components: reconstruction and classification, each with their own weight to regulate their overall contribution to the loss function (reconstruction error is also on a different scale than the cross-entropy loss so the relative weighting terms $\alpha$ and $\beta$ are necessary for balancing contributions of the losses). Training the network consists of learning the correct parametrization $\theta$ (network weights) which minimizes the loss function over the training dataset. Early Stopping was applied on an evaluation dataset to avoid overfitting of the training data. One autoencoder was trained per mouse using a subset of that mouse's first months recording data. The subset was chosen using conservative thresholds on the within-cluster centroid distance of UMAP embedded spike waveforms. This allows to train the autoencoder in a very short time on high-quality spikes without hurting classification results (Extended Data Fig. 8b). It also 
777

778

779

780

781

782

783

784

785

786

787

788

789

790

791

792

793

794

795

796

797

798

3

means the autoencoder's training manifold can be used as a stability verification tool (Fig. $5 \mathrm{f}$ and Extended Data Fig. 8h-k). Indeed, waveforms recorded in the later parts of the recordings with similar shapes as the high-quality training waveforms will fall inside the training manifold.

Aging analysis at the single-neuron level: UMAP coordinates of the representation in Fig. 4 were used to visualize the different neuron trajectories shown in Figure 6 defined by their electrophysiological properties across the adult mouse life recording. Indeed, these UMAP embeddings were used to compute a trajectory graph with the corresponding "pseudo temporal" scale of evolution, with Monocle 3 (https://cole-trapnell-lab.github.io/monocle3). The convex hull delimiting regions spanned by individual neuron time-evolution was calculated using Scipy; Principal Component Analysis (PCA) was used as another form of dimensionality reduction and was calculated per channel and month to view the evolution of spike waveforms per cluster (Extended Data Fig. 6b). Clusters obtained from the same channel were used to obtain cluster centroids per channel for individual recording months along with $2 \mathrm{SD}$ confidence ellipses of corresponding covariance. Confidence ellipses were centered on cluster means while radii were calculated without explicit computation of eigenvalues by using Pearson correlation coefficients and equations (6) and (7):

$$
\sqrt{\lambda_{1}}=\sqrt{1+p}
$$

$$
\sqrt{\lambda_{2}}=\sqrt{1-p}
$$

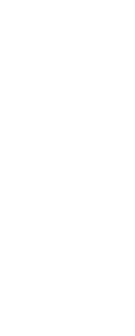


799 Feature trajectory analysis was performed by fitting trajectories, from feature embeddings in a 800 selected 3D feature space of mean feature values per cluster over real recording months. The 801 features chosen for the representation were repolarization slope, peak trough ratio and duration. 802 These were selected based on prior correlation analysis (Extended Data Fig. 9a) to make sure the 803 trajectory was not a degenerate representation of feature evolution because of highly correlated 804 features. Trajectory fittings were constructed using a B-spline interpolation on previously 805 discussed points by first finding the parametric definition of the curve using Scipy splrep function 806 before evaluating the spline using splev function. A cubic $(k=3)$ spline was used with $\mathrm{s}=2$. as the 807 smoothing condition in splrep. 
808 Acknowledgements We acknowledge the discussion and assistance from all Liu Group members,

809 Jane Salant, and Prof. Bence P. Ölveczky. We acknowledge the support from the Harvard

810 University School of Engineering and Applied Sciences' Startup fund and Harvard University

811 Faculty of Arts and Sciences’ Dean's Competitive Fund for Promising Scholarship.

812 Author contributions J.L. and S.Z. conceived and designed the experiments. S.Z., R. L, J. Lee.

813 fabricated and characterized the electrodes. S.Z., Z.L. performed the brain implantation, in vivo

814 recording and histology study. S.Z., X.T., S.P. conducted the data analysis. The manuscript was

815 written by J.L., S.Z., X.T., and S.P.

816 Correspondence and requests for materials should be addressed to J. L.

817 (jia_liu@seas.harvard.edu) 


\section{References}

8191 Gallego, J. A., Perich, M. G., Chowdhury, R. H., Solla, S. A. \& Miller, L. E. Long-term 820 stability of cortical population dynamics underlying consistent behavior. Nat. Neurosci. 23, $821260-270(2020)$.

8222 Steinmetz, N. A. et al. Neuropixels 2.0: A miniaturized high-density probe for stable, long823 term brain recordings. Science 372, eabf4588 (2021).

8243 Dhawale, A. K. et al. Automated long-term recording and analysis of neural activity in 825 behaving animals. eLife 6, e27702 (2017).

8264 Schoonover, C. E., Ohashi, S. N., Axel, R. \& Fink, A. J. P. Representational drift in primary 827 olfactory cortex. Nature 594, 541-546 (2021).

8285 Igarashi, K. M., Lu, L., Colgin, L. L., Moser, M.-B. \& Moser, E. I. Coordination of 829 entorhinal-hippocampal ensemble activity during associative learning. Nature 510, 143$830147(2014)$.

8316 Dhawale, A. K., Wolff, S. B. E., Ko, R. \& Ölveczky, B. P. The basal ganglia control the 832 detailed kinematics of learned motor skills. Nat. Neurosci. 24, 1256-1269 (2021).

8337 Wang, M. et al. Neuronal basis of age-related working memory decline. Nature 476, 210$834 \quad 213(2011)$.

8358 Grady, C. The cognitive neuroscience of ageing. Nat. Rev. Neurosci. 13, 491-505 (2012).

8369 Meng, G. et al. High-throughput synapse-resolving two-photon fluorescence 837 microendoscopy for deep-brain volumetric imaging in vivo. eLife 8, e40805 (2019).

83810 Salatino, J. W., Ludwig, K. A., Kozai, T. D. Y. \& Purcell, E. K. Glial responses to 839 implanted electrodes in the brain. Nat. Biomed. Eng. 1, 862-877 (2017). 
$84011 \mathrm{Ji}, \mathrm{N}$. The Practical and fundamental limits of optical imaging in mammalian brains. $841 \quad$ Neuron 83, 1242-1245 (2014).

$84212 \mathrm{Yu}, \mathrm{K}$. J. et al. Bioresorbable silicon electronics for transient spatiotemporal mapping of 843 electrical activity from the cerebral cortex. Nat. Mater. 15, 782-791 (2016).

84413 Chiang, C.-H. et al. Development of a neural interface for high-definition, long-term 845 recording in rodents and nonhuman primates. Sci. Transl. Med. 12, eaay4682 (2020).

84614 Song, E. et al. Flexible electronic/optoelectronic microsystems with scalable designs for 847 chronic biointegration. Proc. Natl. Acad. Sci.116, 15398-15406 (2019).

84815 Liu, Y. et al. Soft and elastic hydrogel-based microelectronics for localized low-voltage 849 neuromodulation. Nat. Biomed. Eng. 3, 58-68 (2019).

85016 Yin, R. et al. Soft transparent graphene contact lens electrodes for conformal full-cornea 851 recording of electroretinogram. Nat. Commun. 9, 2334 (2018).

85217 Zhang, J. et al. Stretchable transparent electrode arrays for simultaneous electrical and 853 optical interrogation of neural circuits in vivo. Nano Lett. 18, 2903-2911 (2018).

85418 Liu, J. et al. Syringe-injectable electronics. Nat. Nanotechnol. 10, 629-636 (2015).

85519 Yang, X. et al. Bioinspired neuron-like electronics. Nat. Mater. 18, 510-517 (2019).

85620 Fu, T.-M. et al. Stable long-term chronic brain mapping at the single-neuron level. Nat. $857 \quad$ Methods 13, 875-882 (2016).

85821 Guan, S. et al. Elastocapillary self-assembled neurotassels for stable neural activity 859 recordings. Sci. Adv. 5, eaav2842 (2019).

86022 Kim, T.-i. et al. Injectable, cellular-scale optoelectronics with applications for wireless $861 \quad$ optogenetics. Science 340, 211-216 (2013). 
86223 Musk, E. An integrated brain-machine interface platform with thousands of channels. $J$. Med. Internet. Res.21,e16194 (2019)

86424 He, F. et al. Multimodal mapping of neural activity and cerebral blood flow reveals long865 lasting neurovascular dissociations after small-scale strokes. Sci. Adv. 6, eaba1933 (2020). mechanics and mechanical properties of mouse brain tissue at micrometer scales. IEEE Trans. Biomed. Eng. 56, 45-53 (2008).

86926 McInnes, L., Healy, J. \& Melville, J. Umap: Uniform manifold approximation and 870 projection for dimension reduction. Preprint at https://arxiv.org/abs/1802.03426 (2018).

87127 Liu, W. et al. A survey of deep neural network architectures and their applications. Neurocomputing 234, 11-26 (2017).

87328 Jeong, J.-W. et al. Wireless optofluidic systems for programmable in vivo pharmacology $874 \quad$ and optogenetics. Cell 162, 662-674 (2015).

87529 Seo, K. J. et al. Transparent, flexible, penetrating microelectrode arrays with capabilities of single-unit electrophysiology. Adv. Biosyst. 3, 1800276 (2019).

87730 Xie, C. et al. Three-dimensional macroporous nanoelectronic networks as minimally invasive brain probes. Nat. Mater. 14, 1286-1292 (2015).

87931 Kozai, T. D. Y. et al. Ultrasmall implantable composite microelectrodes with bioactive surfaces for chronic neural interfaces. Nat. Mater. 11, 1065-1073 (2012).

88132 Lu, L. et al. Soft and MRI compatible neural electrodes from carbon nanotube fibers. Nano Lett. 19, 1577-1586 (2019).

88333 Guitchounts, G., Markowitz, J. E., Liberti, W. A. \& Gardner, T. J. A carbon-fiber electrode 884 array for long-term neural recording. J. Neural Eng. 10, 046016 (2013). 
88534 Rousche, P. J. et al. Flexible polyimide-based intracortical electrode arrays with bioactive capability. IEEE Trans. Biomed. Eng. 48, 361-371 (2001).

88735 Minev, I. R. et al. Electronic dura mater for long-term multimodal neural interfaces. Science 347, 159-163 (2015). implants. J. Neural Eng. 16, 026013 (2019).

89137 Barrese, J. C. et al. Failure mode analysis of silicon-based intracortical microelectrode arrays in non-human primates. J. Neural Eng. 10, 066014 (2013).

89338 Chaure, F. J., Rey, H. G. \& Quiroga, R. Q. A novel and fully automatic spike-sorting implementation with variable number of features. J. Neurophysiol. 120, 1859-1871 (2018).

89539 Viswanathan, P. \& Nieder, A. Visual receptive field heterogeneity and functional 896 connectivity of adjacent neurons in primate frontoparietal association cortices. J. Neurosci.

89840 Jia, X. et al. High-density extracellular probes reveal dendritic backpropagation and 899 facilitate neuron classification. J. Neurophysiol. 121, 1831-1847 (2019).

$90041 \quad$ Flurkey, K., M. Currer, J. \& Harrison, D. E. The Mouse in Biomedical Research Ch.20 901 (eds James G. Fox et al.) 637-672 (Academic Press, Burlington, 2007).

90242 Zhao, S. et al. Full activation pattern mapping by simultaneous deep brain stimulation and fMRI with graphene fiber electrodes. Nat. Commun. 11, 1788 (2020).

90443 Schmitzer-Torbert, N., Jackson, J., Henze, D., Harris, K. \& Redish, A. D. Quantitative 905 measures of cluster quality for use in extracellular recordings. Neuroscience 131, 1-11 (2005). 
90744 Rousseeuw, P. J. Silhouettes: A graphical aid to the interpretation and validation of cluster 908 analysis. J. Comput. Appl. Math. 20, 53-65 (1987).

90945 Wang, Q., Webber, R. M. \& Stanley, G. B. Thalamic synchrony and the adaptive gating of 910 information flow to cortex. Nat. Neurosci. 13, 1534-1541 (2010).

$91146 \quad$ Breiman, L. Random Forests. Mach. Learn. 45, 5-32 (2001).

91247 Cao, J. et al. The single-cell transcriptional landscape of mammalian organogenesis. Nature $913 \quad 566,496-502(2019)$.

$914 \quad 48$ Zhao, S. et al. Graphene encapsulated copper microwires as highly MRI compatible neural 915 electrodes. Nano Lett. 16, $7731-7738$ (2016).

91649 Traag, V. A., Waltman, L. \& van Eck, N. J. From Louvain to Leiden: guaranteeing well917 connected communities. Sci. Rep. 9, 5233 (2019).

918

919

920

921

922

923

924

925

926

927 
a

Deposit $\mathrm{Ni}$

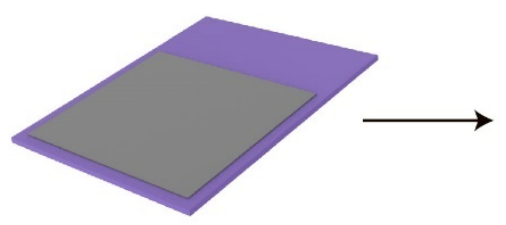

$\mathbf{f}$ Spin-coated dextran
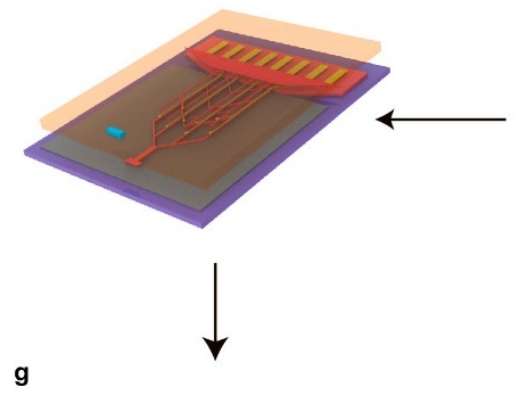

Pattern SU-8 shuttle

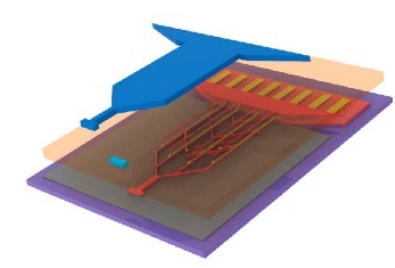

b

Pattern bottom SU-8

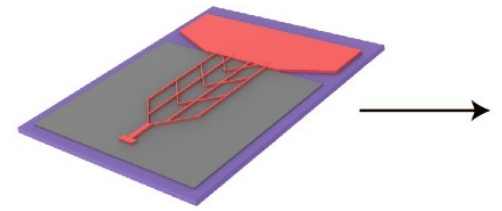

e

Pattern anchor

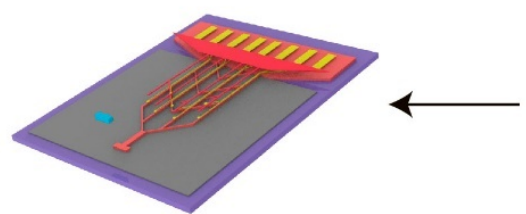

$\mathrm{Si} / \mathrm{SiO}_{2}$ chip

Au interconnects /Pt sensors

Dextran c

Pattern Au interconnects and $\mathrm{Pt}$ sensors

d

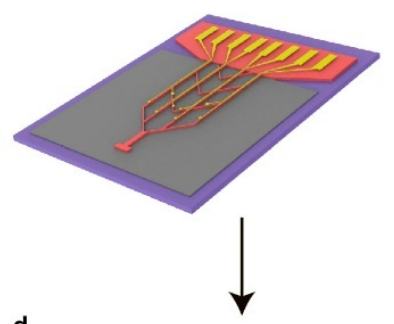

Pattern top SU-8

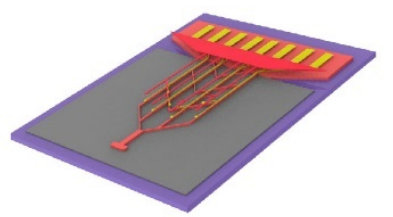

$\mathrm{Ni}$

Bottom/Top SU-8

SU-8 anchor

SU-8 shuttle
Extended Data Fig. 1 | Schematics show the stepwise fabrication of monolithically integrated tissue-level flexible mesh nanoelectronics. a, A $\mathrm{Ni}$ sacrificial layer (grey) was defined by photolithography and deposited through thermal evaporation on the $\mathrm{Si} / \mathrm{SiO}_{2}$ wafer (purple). b, SU82000.5 bottom passivation layer (red) was defined by photolithography. c, Cr/Au interconnects and Pt microelectrodes (yellow) were sequentially defined by photolithography and deposited through electron beam (e-beam) evaporation on the top of the SU-8 passivation layer. d, SU-8 2000.5 top passivation was defined by photolithography (red). e, SU-8 2025 anchor was defined by photolithography (cyan). f, Dextran sacrificial layer (pink) was spin coated. g, SU-8 2025 shuttle was defined by photolithography (navy). 
a

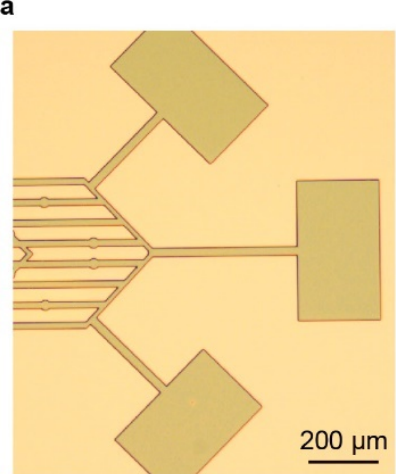

e

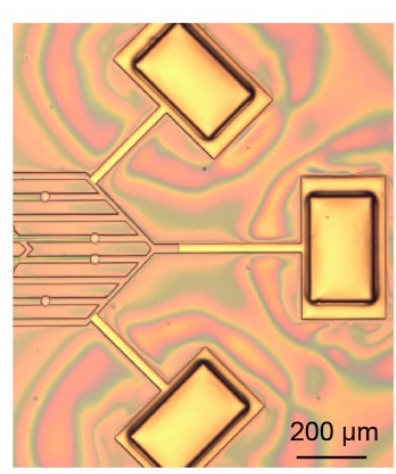

b
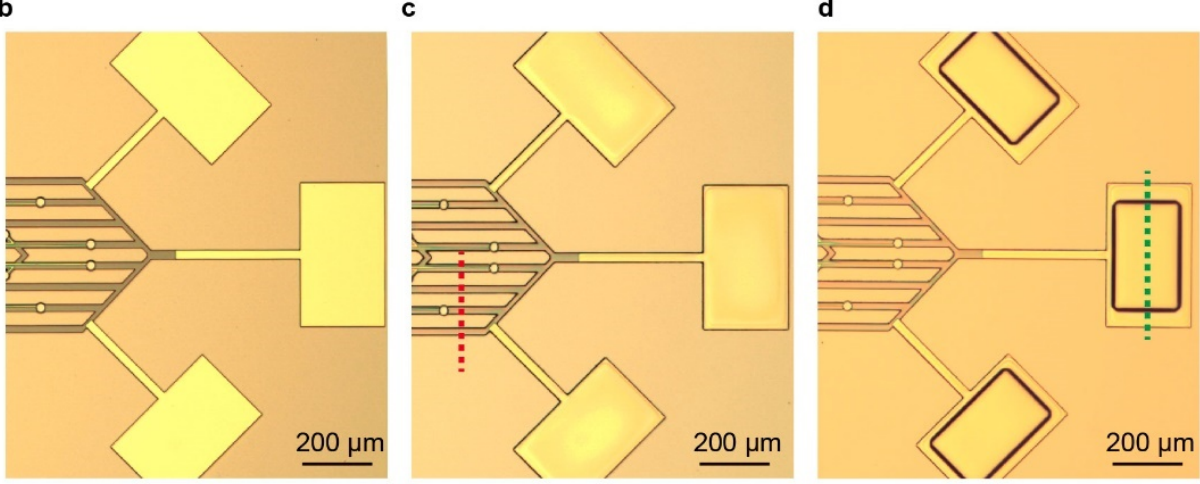

f

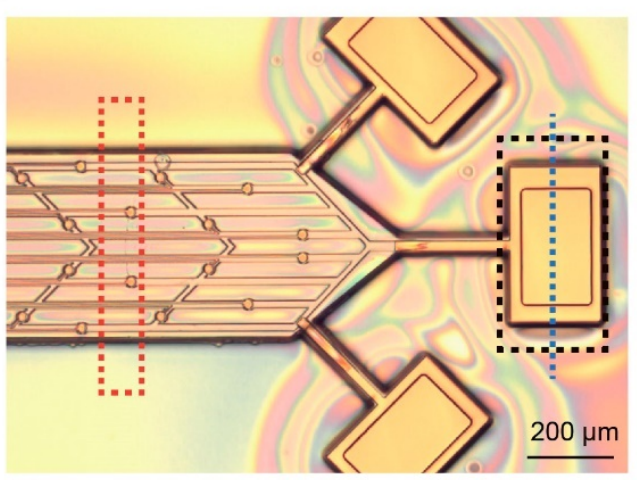

g

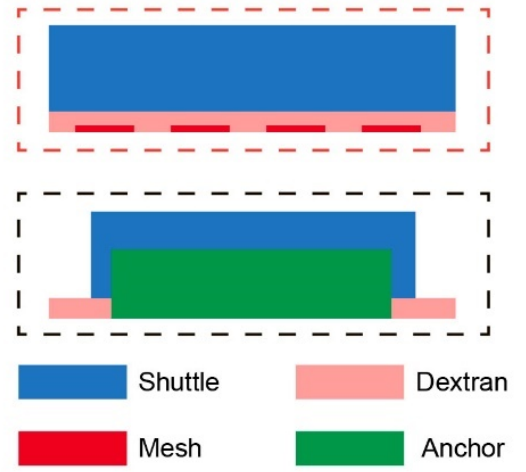

h

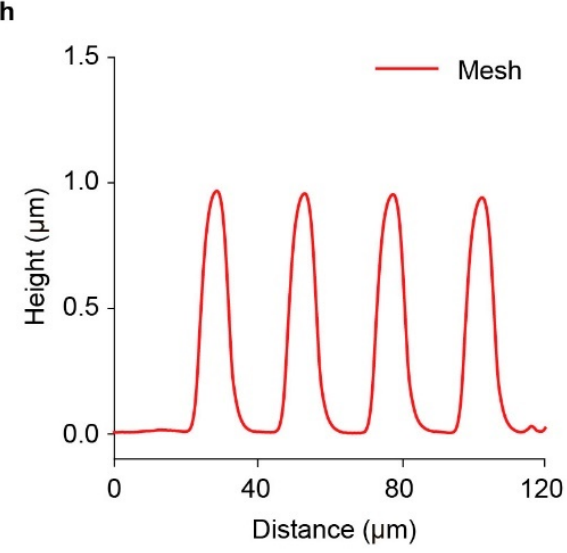

$\mathbf{k}$

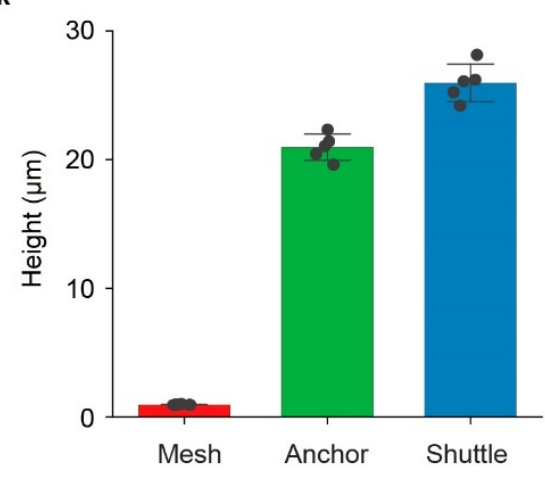

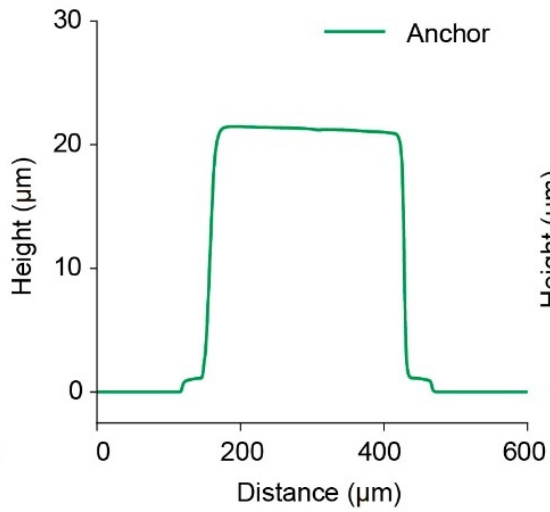

I

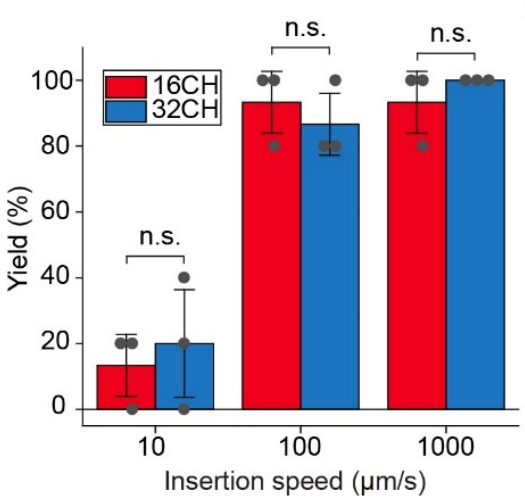

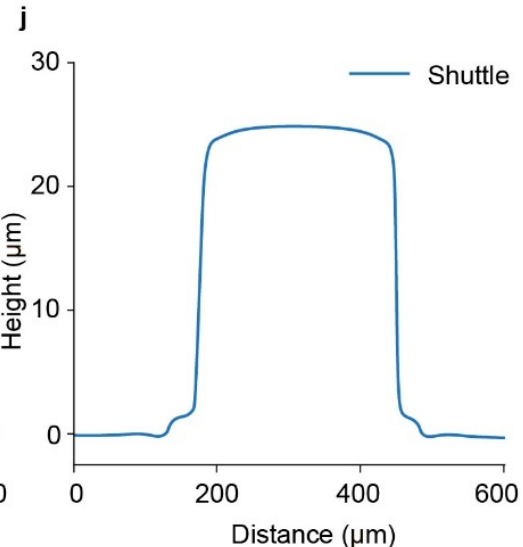

m

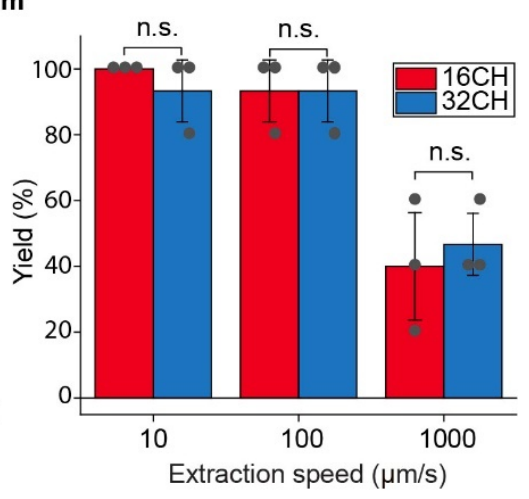


940 Extended Data Fig. 2 | Anchor structures for controllable implantation. a-f, Optical images 941 illustrating each step of the fabrication corresponding with Extended Data Fig. 1b-g, respectively. $942 \mathrm{~g}$, Schematics showing the cross-section of the monolithically integrated mesh nanoelectronics at 943 the red and black dashed boxes highlighted regions in (f), respectively. $\mathbf{h}-\mathbf{j}$, Contact profilometer 944 measurements along with the open mesh structure in (c, red dashed line), anchor structure in (d, 945 green dashed line), and shuttle structure in (f, blue dashed line). $\mathbf{k}$, Statistical summary of the 946 thickness of the open mesh, anchor, and shuttle layer structures $(n=5)$. $\mathbf{l}, \mathbf{m}$, Insertion (l) and 947 extraction $(\mathbf{m})$ yield of 16-channel and 32-channel mesh nanoelectronics with different speeds (n.s.: 948 not significant, two-tailed unpaired $t$ test, $n=3$ times experiment, each time include 5 949 insertion/extraction). 
bioRxiv preprint doi: https://doi.org/10.1101/2021.10.29.466524; this version posted November 1, 2021. The copyright holder for this preprint (which was not certified by peer review) is the author/funder. All rights reserved. No reuse allowed without permission.

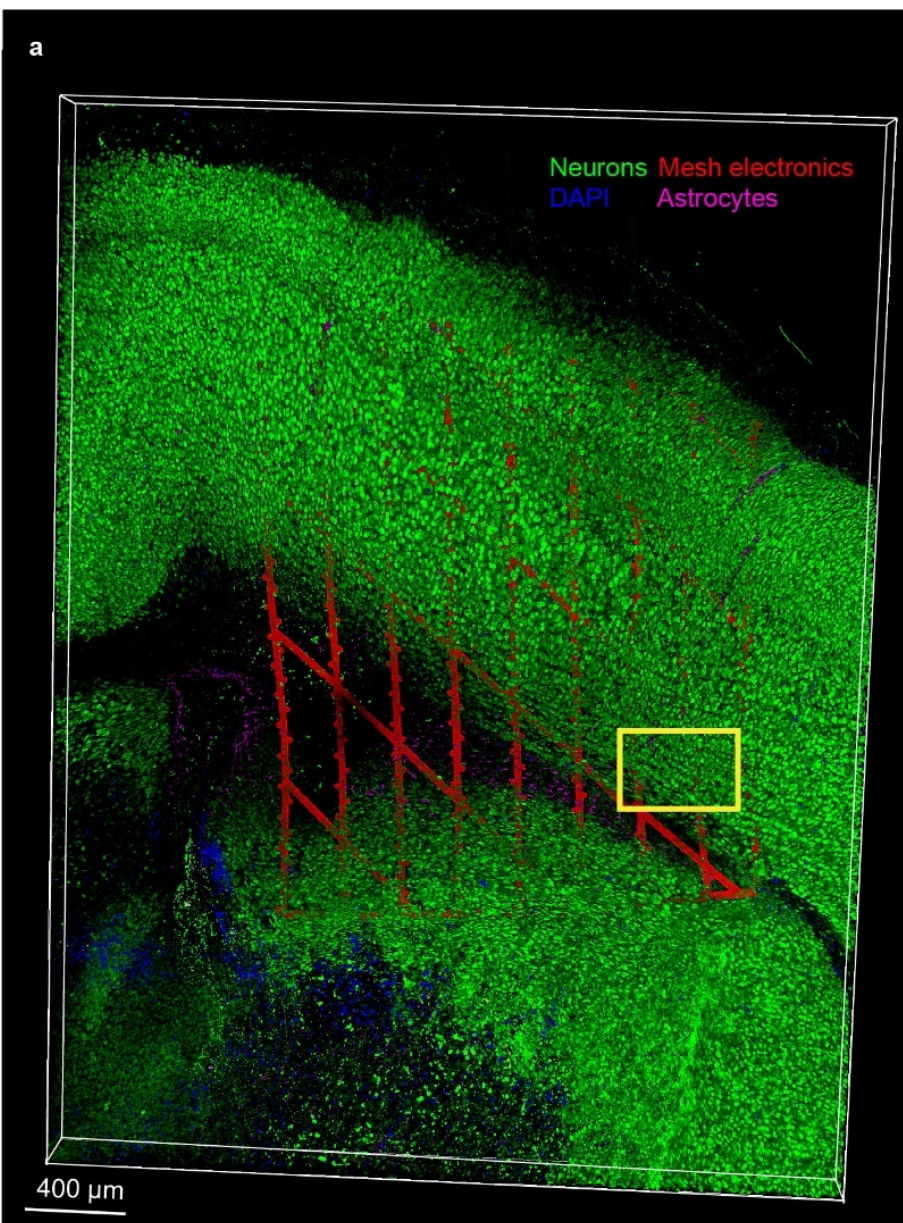

b

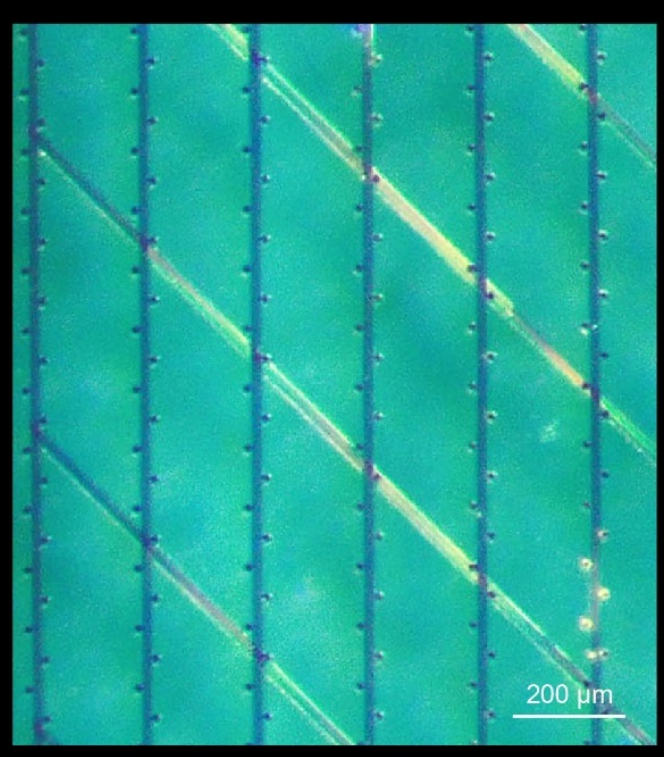

c

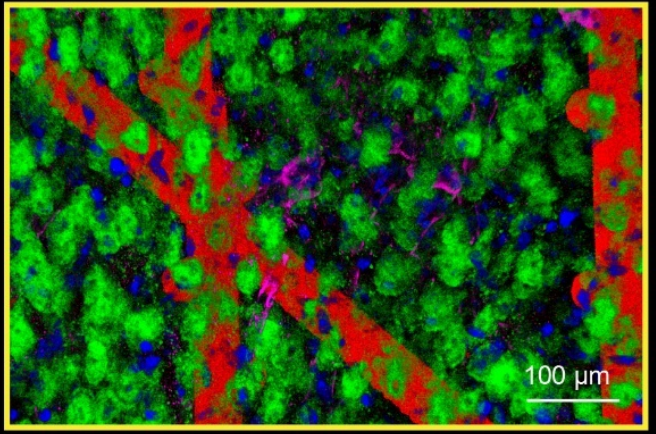

d

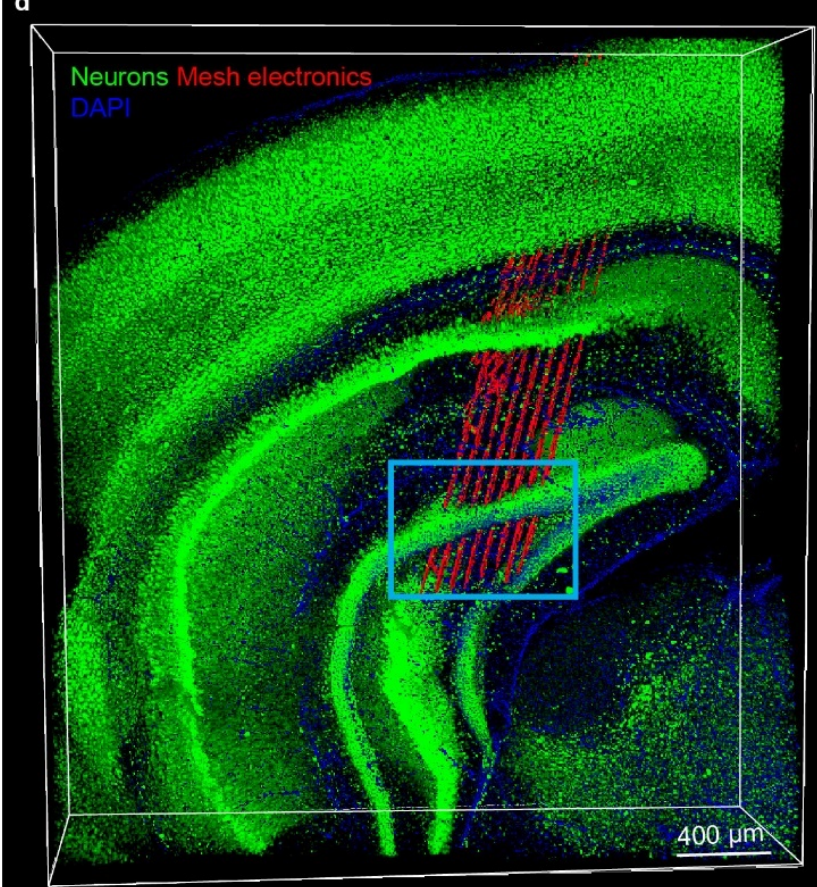

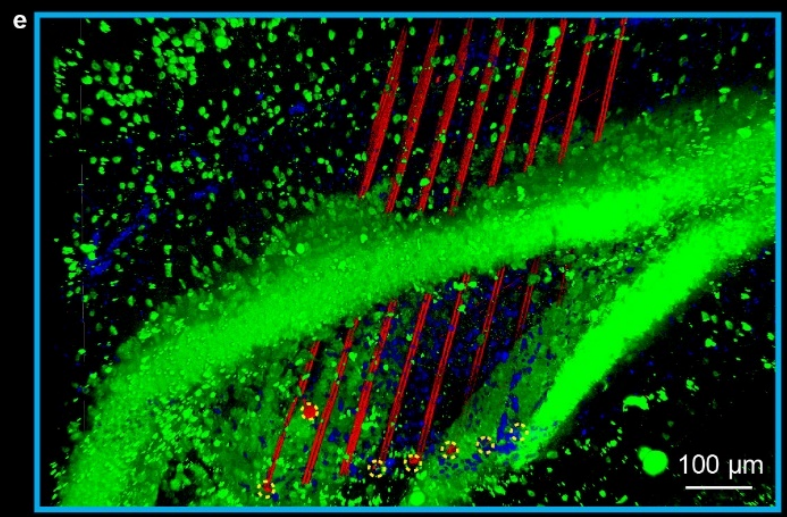

f

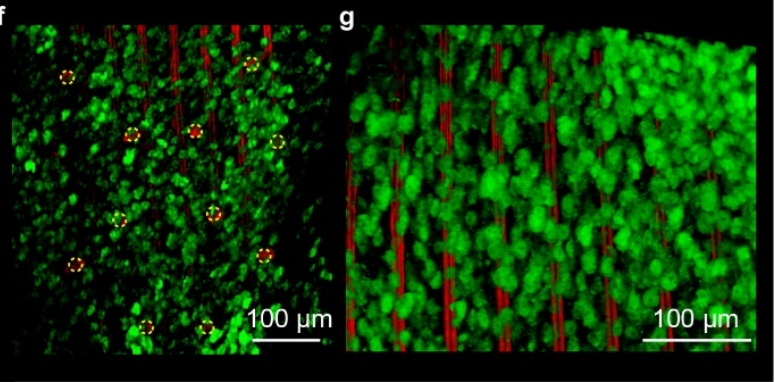


951 Extended Data Fig. 3 | Independent replicates of mesh nanoelectronics with different sizes 952 were implanted in mice brains. a, 3D reconstructed confocal fluorescence imaging of neuron 953 (green), nuclei (blue), and astrocytes (purple) with 1024-channel mesh nanoelectronics (red) 954 sustaining their open mesh structure across multiple brain regions at 6-week post-implantation. b, 955 Representative photograph illustrates the high density, 1024-channel mesh nanoelectronics. c, 956 Zoom-in views of the regions highlighted by yellow (c) box in (a). d, A 3D reconstructed interface 957 of neurons (green), nuclei (blue) with shape maintained 16-channel mesh electronic (red) at 6958 week post-implantation. The mesh electronic was across the cortex and hippocampus with a 959 designed 30-degree angle corresponding to the dorsal-ventral direction. e, A zoom-in view of the 960 hippocampus region highlighted by the cyan box in (a). f, g, Neuron interpenetration inside the 961 subcellular electrode, individual electrodes are indicated by yellow dashed circles in (e) and (f) 962 and opening mesh structure (g). These results show neuron interpenetration inside the opening 963 mesh and minimal astrocyte increases at the surface and interior of mesh, and thus demonstrate 964 the reproducibility of these results in Fig. 2. 
a

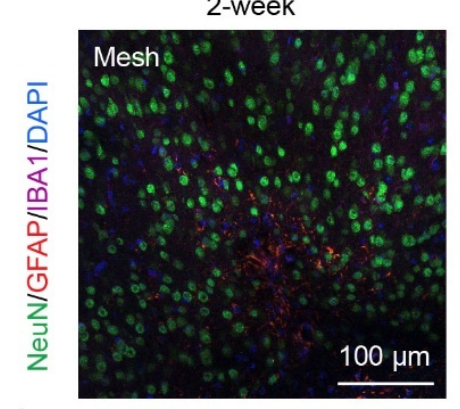

b

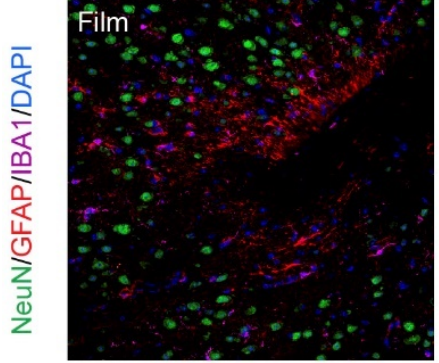

6-week
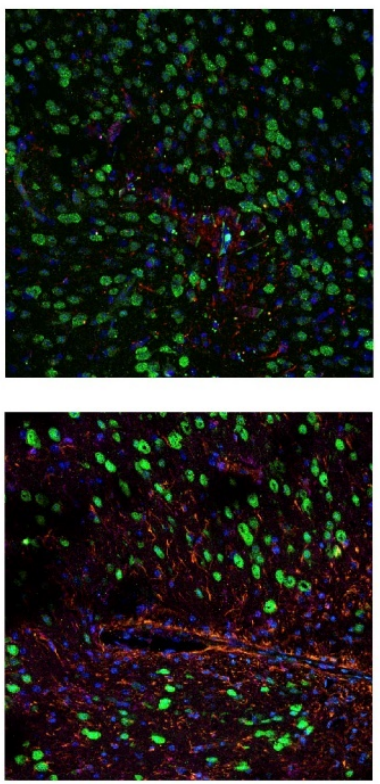

12-week
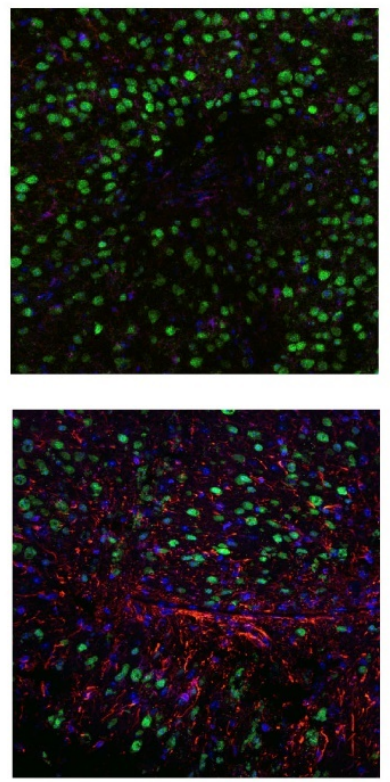

1-year
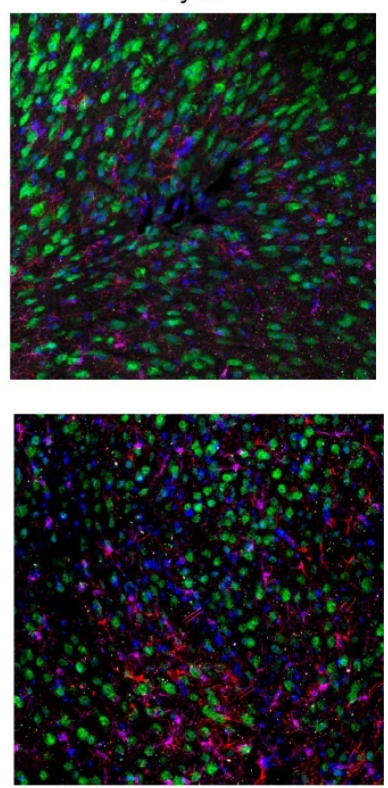

967 Extended Data Fig. 4 | Time-dependent histology studies of brain tissue reaction to $\sim 1 \boldsymbol{\mu m}-$ 968 thick open mesh/thin-film nanoelectronics. a, b, Representative immunofluorescence images of 969 brain tissue reaction following 2-week, 6-week, 12-week, and 1-year post-implantation of a mesh 970 (a) and a thin-film (b) nanoelectronics from the contralateral hemisphere. The tissue was labeled 971 for astrocytes (red), microglia (purple), neurons (green), and nuclei (blue). Time-dependent 972 histology studies have been repeated on $n=5$ independent samples for each time point, with 973 statistical analyses shown in Fig. 2f-i. 
a

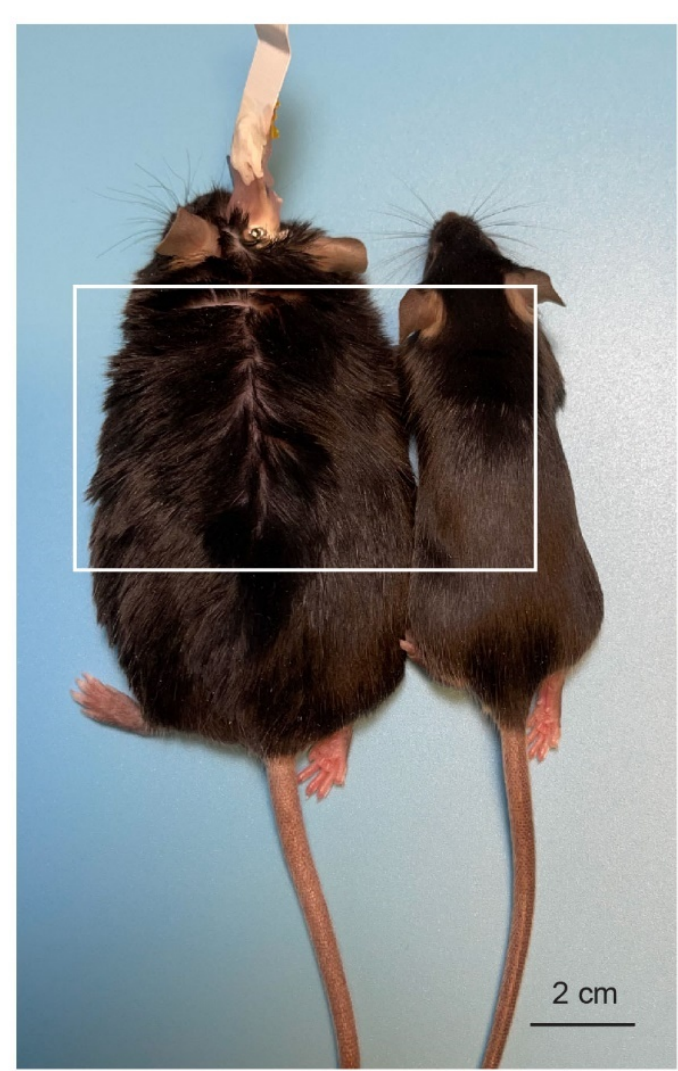

b

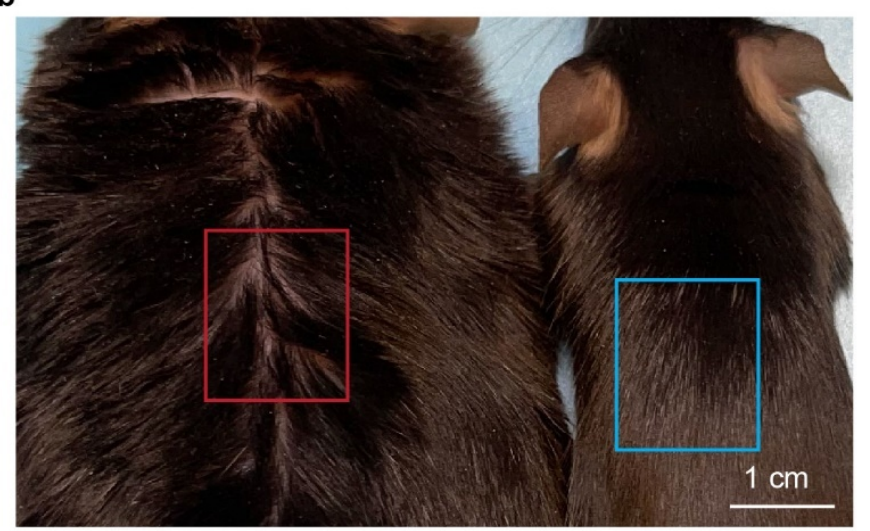

c
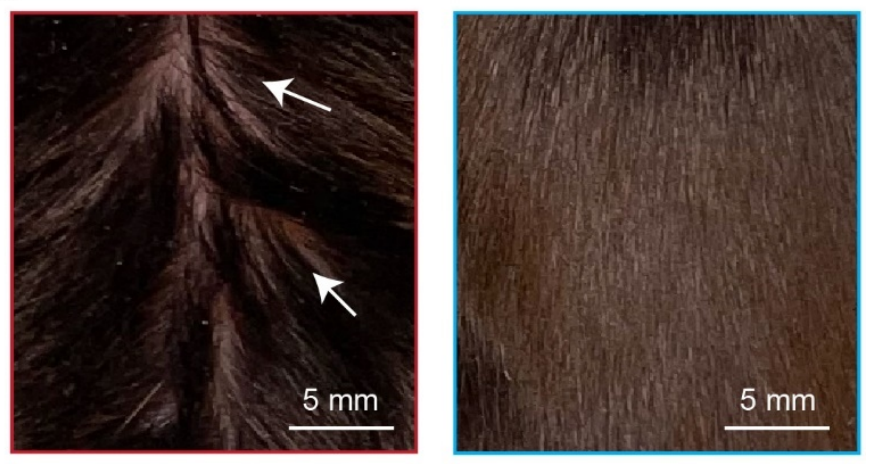

d

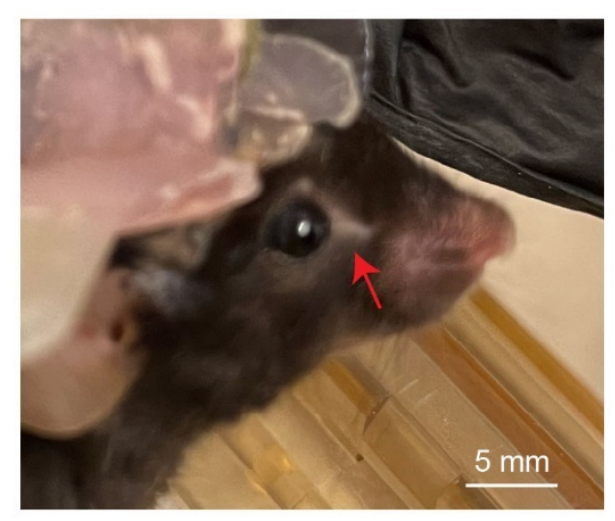

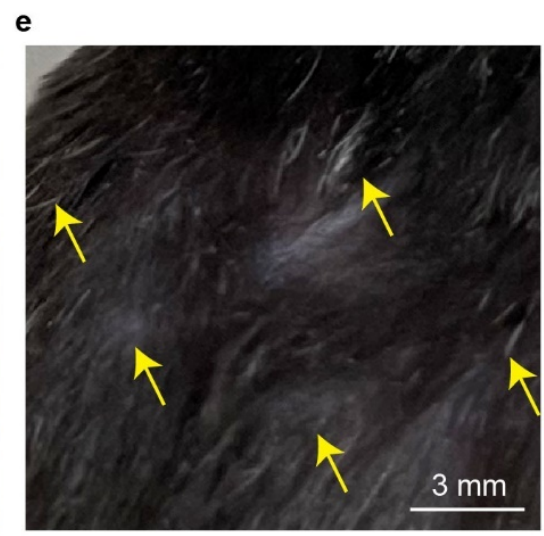

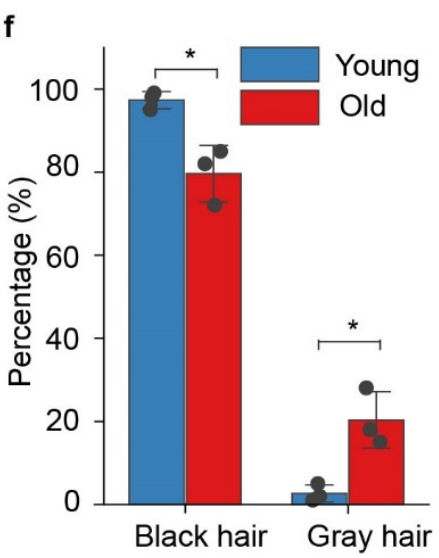

Extended Data Fig. 5 | Aged mice characterization. a, Representative photograph showing the old mouse of weight gain (18 months) with tissue-like mesh nanoelectronics implant (left) compared with the mature adult mouse ( 5 months, right). b, Zoom-in views of the regions highlighted by white boxes in (a). c, Zoom-in views of the thinning hair (white arrows) of aged mouse and glossy brown fur of mature adult mouse highlighted by red and blue boxes in (b). d-e, Representative photograph showing the barbering around eyes (d, red arrow), grey and thinning fur in the dorsal back skin (e, yellow arrows) of the aged mouse (18 months) with mesh nanoelectronics implant. Statistical analysis reveals that significantly increased gray hairs in dorsal back skin in old-aged mice ( ${ }^{*} p<0.05$, two-tailed unpaired $t$ test, $n=3$ ). 

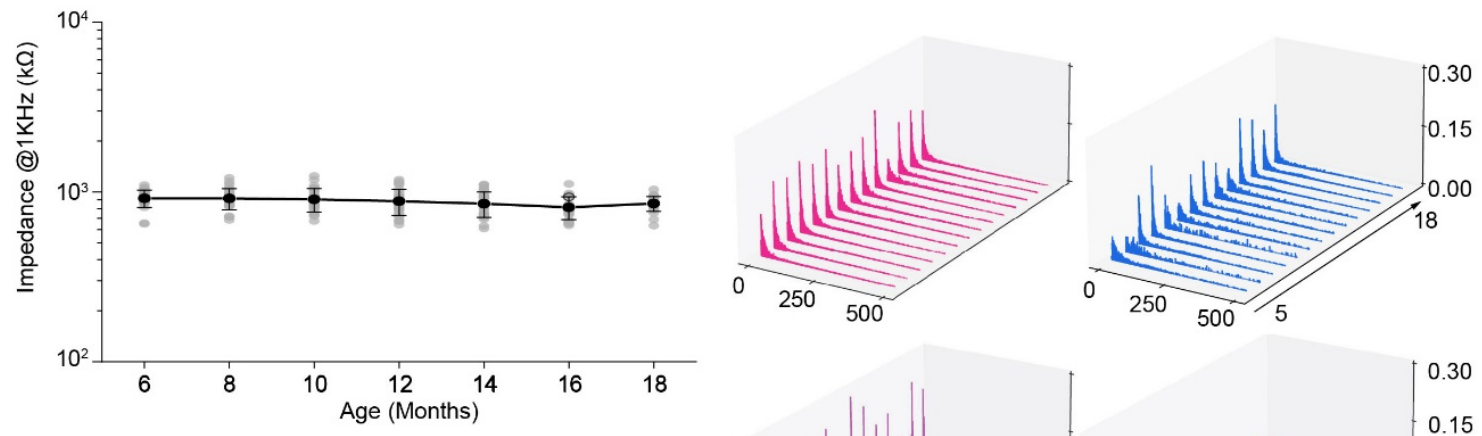

b
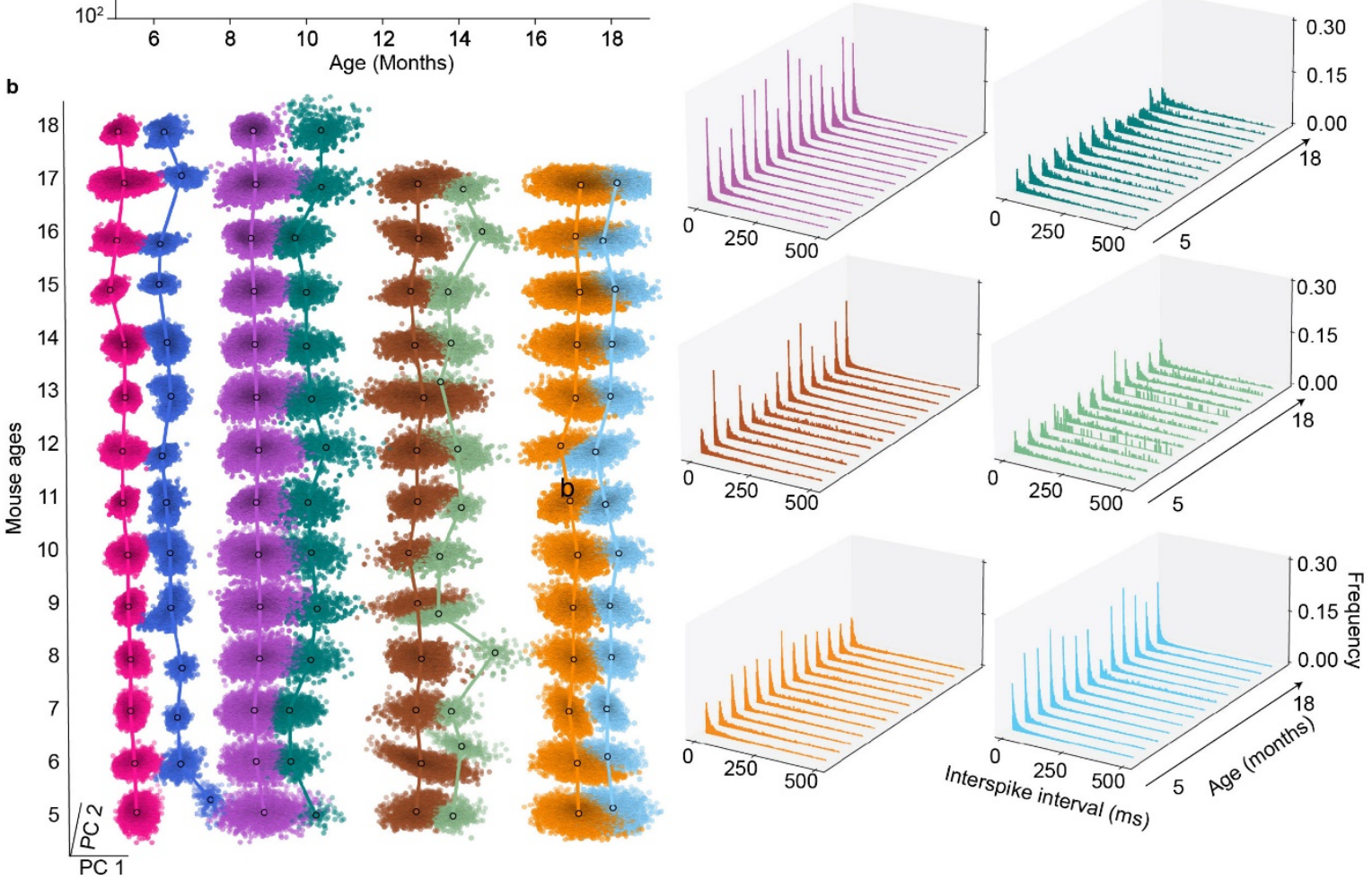

Extended Data Fig. 6 | Long-term stable recording characterization. a, Time-dependent electrode interfacial impedance at $1 \mathrm{kHz}$ measured by the Cereplex Direct (Blackrock Microsystems, USA). Data represented mean \pm SD, individual data points are overlaid. The electrode interfacial impedances exhibited relatively constant values of $920.2 \pm 107.2 \mathrm{k} \Omega$ vs. 857.2 $\pm 85.7 \mathrm{k} \Omega$ (mean $\pm \mathrm{SD}, n=30)$ at months $6 \mathrm{vs} .18$. b, Time evolution of representative single-unit spikes clustered by Leiden clustering. The $\mathrm{x}$ - and y-axes denote the first and second PC dimensions, respectively, and the z-axis denotes mouse age in months. Dimension-reduced clusters associated with a single unit are shown (Fig. 4a) using the same color. These data show stable clusters with nearly constant positions in the first and second principal component plane (PC1-PC2) over the entire recording period from 5 months to 18 months. c, Time evolution of interspike interval (ISI) histograms of representative neurons identified in Fig. 4 from 5 months to 18 months. The x- and $y$-axes denote the time between subsequent action potentials of spontaneous firing neuron, and mouse age in months, respectively, and the z-axis denotes frequency. Bin size, $2 \mathrm{~ms}$. 
a

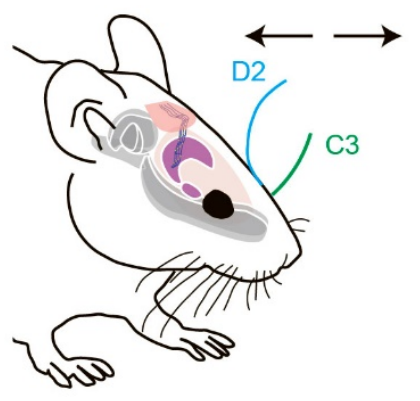

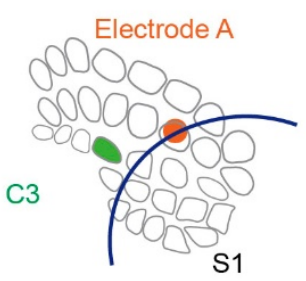

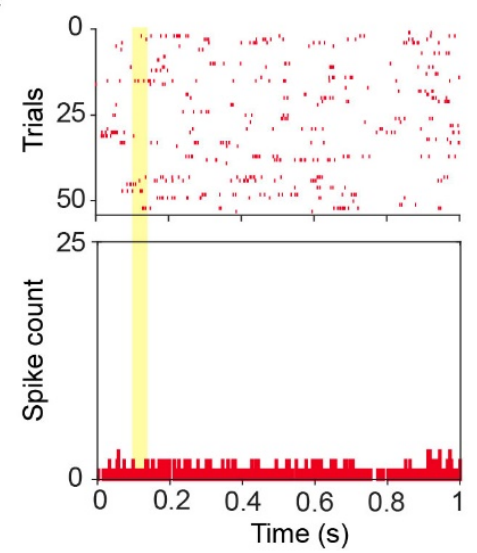

d

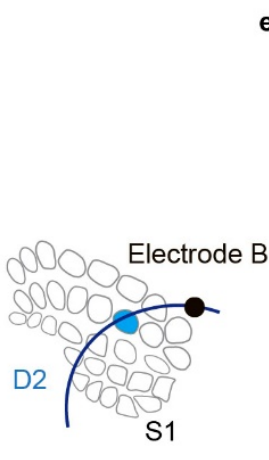

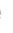

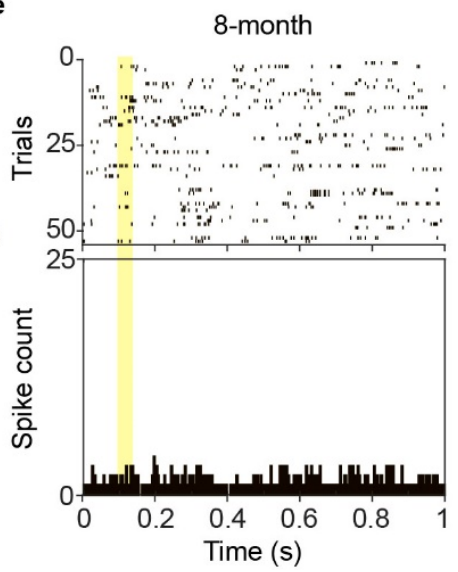

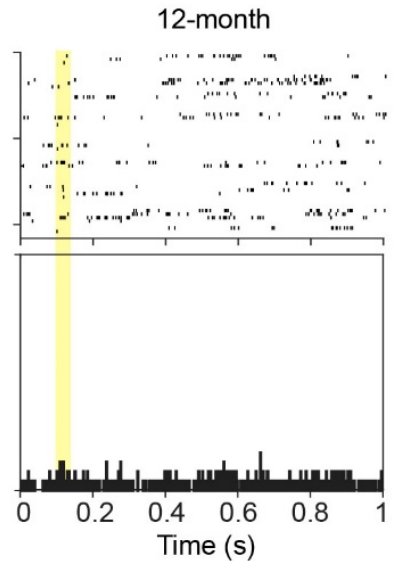

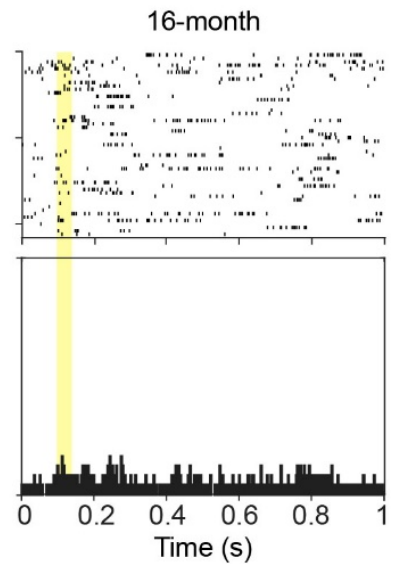

Extended Data Fig. 7 | Validation of whisker-related activity using sham stimulation. a, Schematic diagram of whisker deflection. Individual vibrissa was deflected in the rostral-caudal plane using a computer-controlled piezoelectric bending actuator. D2 and C3 whiskers are labeled in blue and green, respectively. b, Schematic diagram of whisker barrel and electrode arrangement in S1. C3 whisker barrel and electrode A position is labeled in green and orange, respectively. c, Raster plot and peri-stimulus time histogram (PSTH, $1 \mathrm{~ms}$ bin size) of electrode A show no observable spiking activities when applied to the C3 whisker at 8 months. d, D2 whisker and electrode B position are labeled in blue and black in S1 barrel field, respectively. e, Raster plot and PSTH (1 ms bin size) of electrode B show no observable spiking activities when applied to the D2 whisker from 8 months to 16 months. 


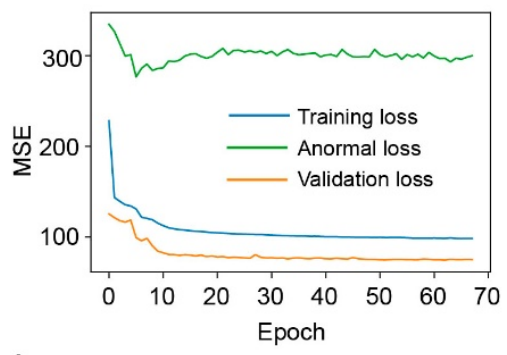

d

Original

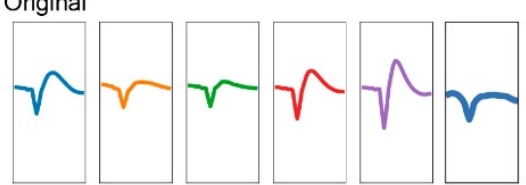

Reconstructed
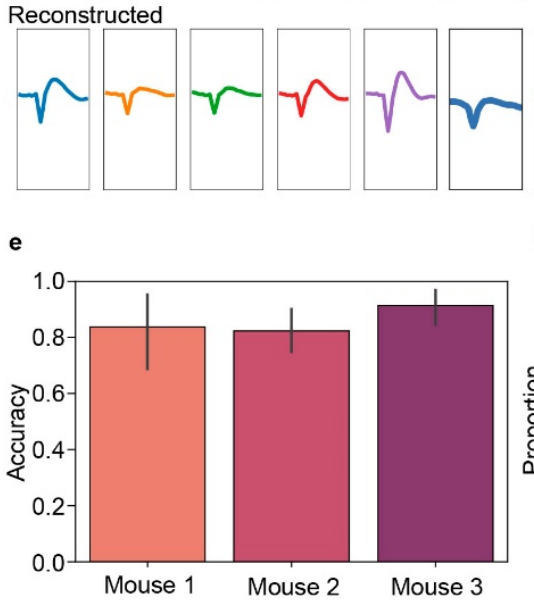

h
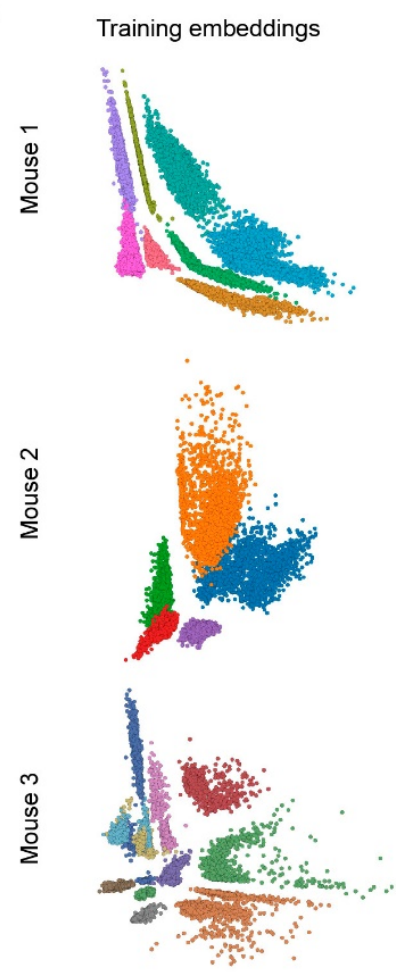

i b

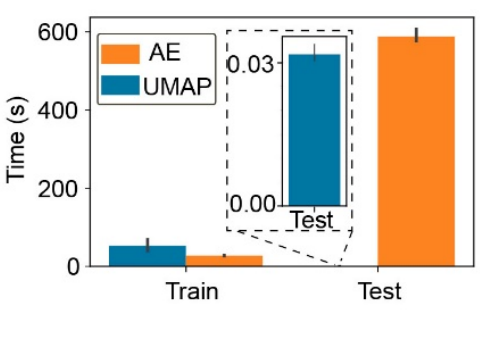

c

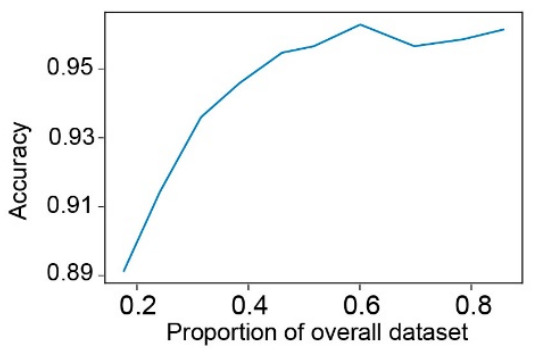

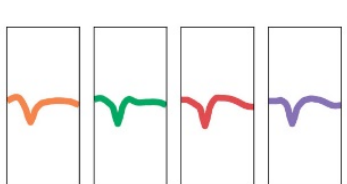
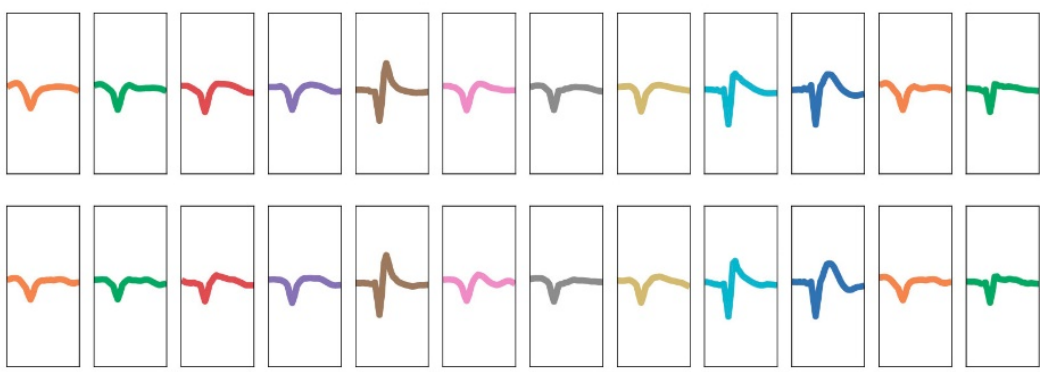

f

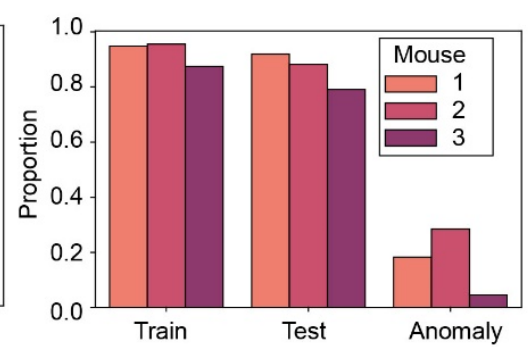

g

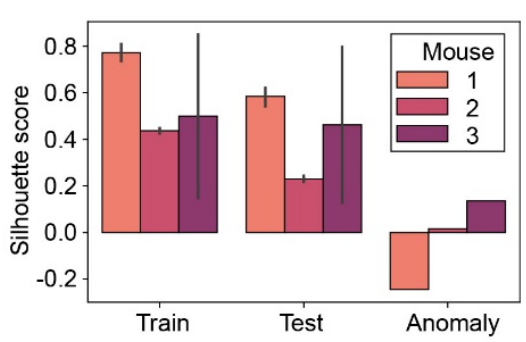

Training manifold

j Testing with noise rejection

k Drifting manifold
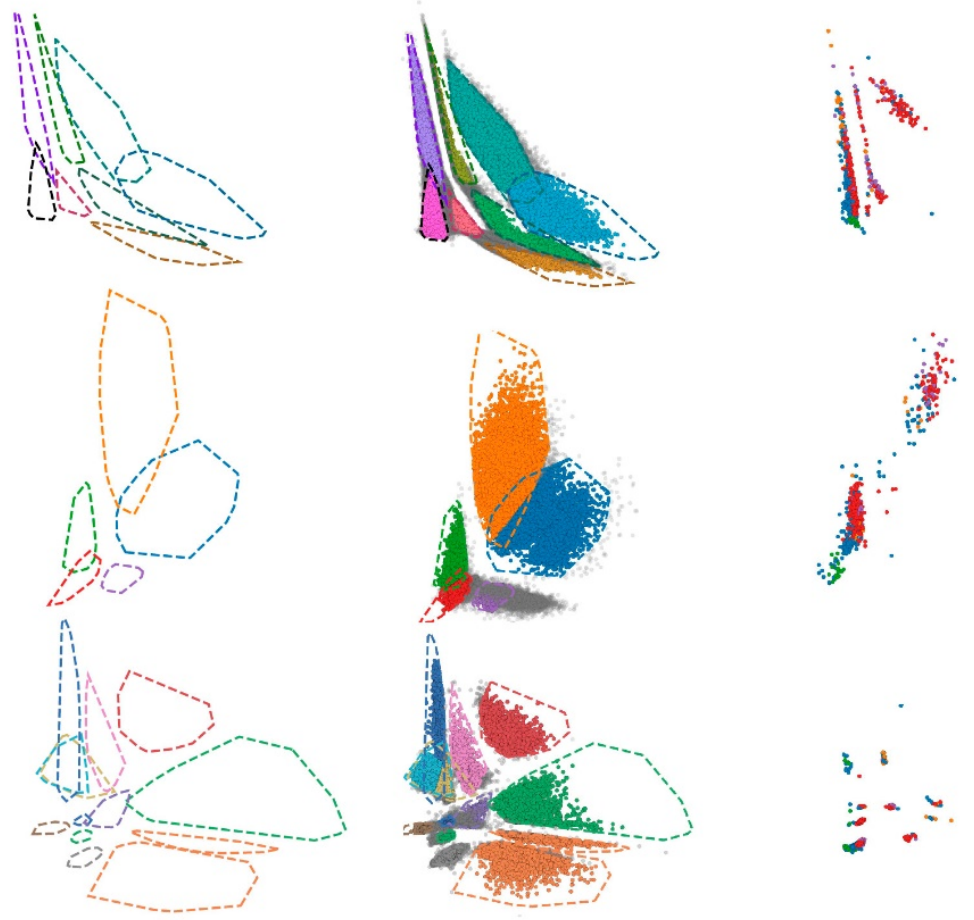
1011 Extended Data Fig. 8 | Autoencoder-based spike sorting and stability analysis a, 1012 Representative training, validation, and drift loss curves obtained during the training process of 1013 one of the autoencoders. b, Time performance comparison of autoencoder vs computational 1014 pipeline allowing for dimensionality reduction and classification (UMAP and a Random Forest 1015 classifier trained on UMAP embeddings). Tests were performed using a single CPU with Intel i5, 10168 cores@4.1GHz. The autoencoder has a significant time advantage for inference. c, Overall 1017 accuracy on the testing dataset as a function of the proportion of the overall dataset used for 1018 training the network. d, Original and reconstructed average neuron waveforms for the second and third mice data. Original waveforms on the top row are colored by within-mouse neuron labels (the first 5 neurons from the left are from mouse 2) while reconstructed waveforms on the bottom row are colored by autoencoder classifier predicted labels. Reconstruction and classification are near-perfect. e, Bar plot of per-mouse classification accuracy calculated for each neuron label class. f, Proportion of spikes kept for each dataset when using MSE-based thresholds for drift detection (Fig. 5d). g, Silhouette score calculated using autoencoder embeddings of training, testing and drift datasets with associated true neuron labels. The training and testing scores reflect the emergent latent space cluster separability for observed neurons while the anomalous scores highlight the poor separability of previously unseen embedded neuron waveforms. $\mathbf{h}, \mathbf{i}, \mathbf{j}$, Stability verification process illustrated for all three mice: visualizing training latent space embeddings (dots colored by their true neuron label), calculating training manifold boundaries, and finally applying these boundaries to quantify the proportion of testing dataset spikes latent embeddings lying inside the predicted neuron's training manifold. k, Visualization of the latent embeddings of drift spikes. Dots are colored by their true neuron labels; mixed colors within clusters showed poor ability to separate different neurons from the drift dataset, in accordance with $(\mathbf{g})$. 
bioRxiv preprint doi: https://doi.org/10.1101/2021.10.29.466524; this version posted November 1, 2021. The copyright holder for this preprint (which was not certified by peer review) is the author/funder. All rights reserved. No reuse allowed without permission.

a

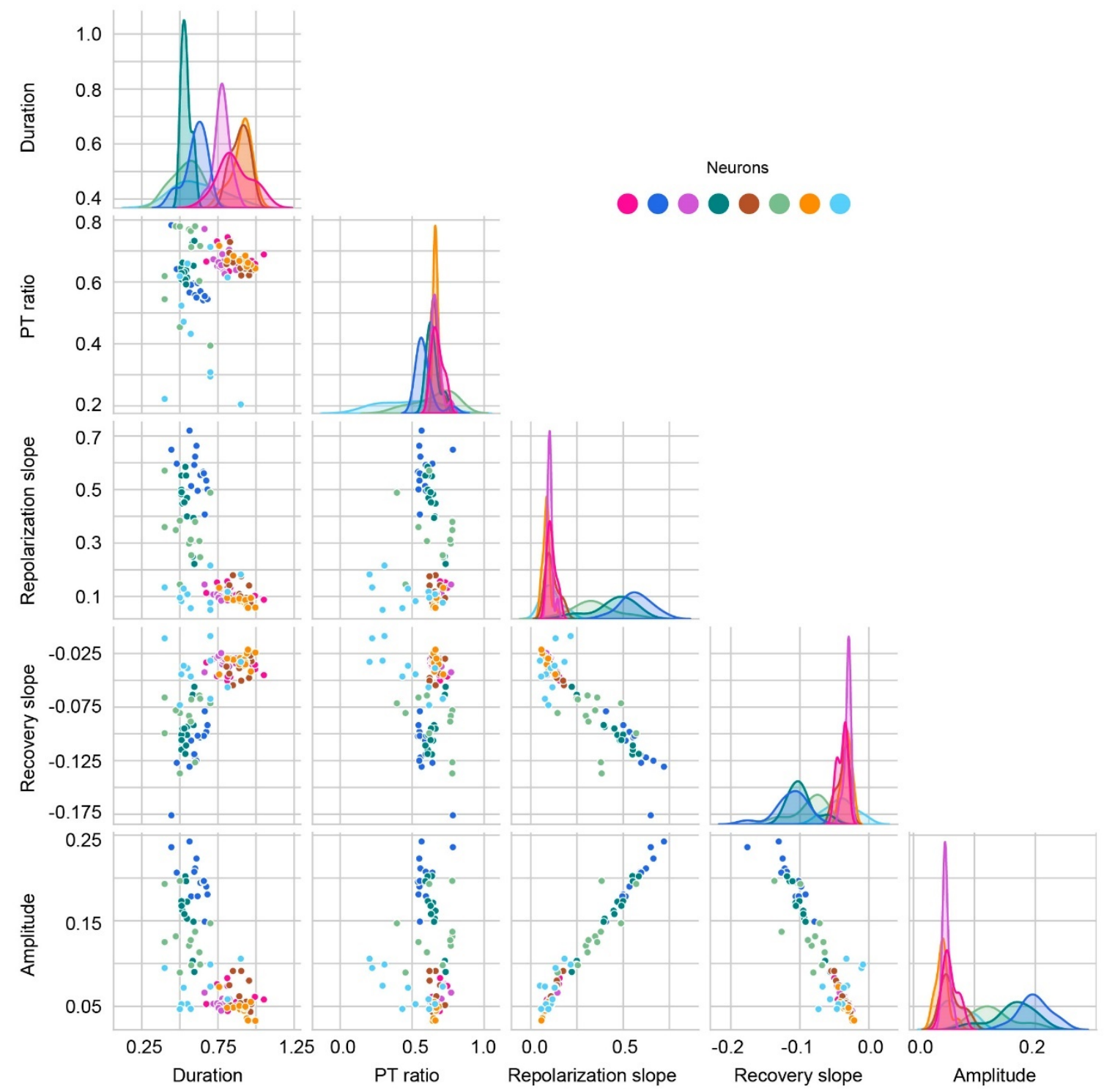

b

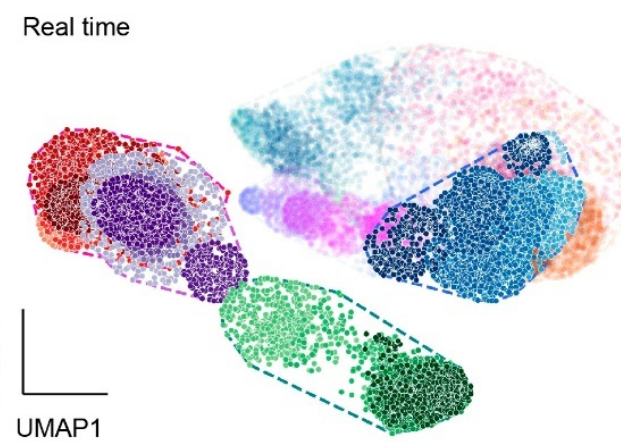

c

Pseudotime

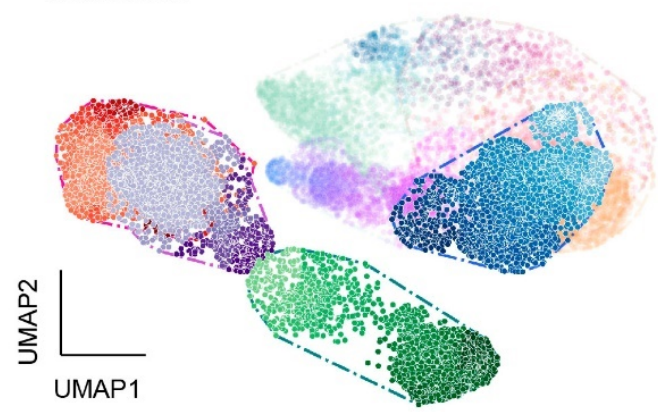

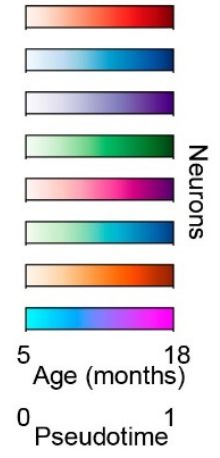


1035 Extended Data Fig. 9 | Feature selection and pseudo time analysis for potential aging1036 associated analysis a, Pairplot of 5 AllenSDK selected features shown in Fig. 4e-h. Dots represent 1037 mean values of paired features calculated over neuron clusters over the entire recording period. 1038 Diagonal subplots show neuron feature univariate distributions using kernel density estimators. b, 1039 c, Real time (b) and pseudotime (c) comparison of UMAP embedding time-evolution for neurons 1040 in Fig. 4. The $\mathrm{x}$ - and $\mathrm{y}$ axes denote the first and second UMAP dimensions, respectively. Each 1041 gradient color-coded cluster represents a distinct neuron in Fig. 4. The color bars show the mouse 1042 age time points from 5 to 18 months and the corresponding pseudo time from 0 to 1 . Highlighted 1043 dots correspond to the representative neurons used in the aging analysis of Fig. 6. Delimiting lines are the convex hull of a neuron's UMAP embedding time-evolution. 
bioRxiv preprint doi: https://doi.org/10.1101/2021.10.29.466524; this version posted November 1, 2021. The copyright holder for this preprint (which was not certified by peer review) is the author/funder. All rights reserved. No reuse allowed without permission.

a
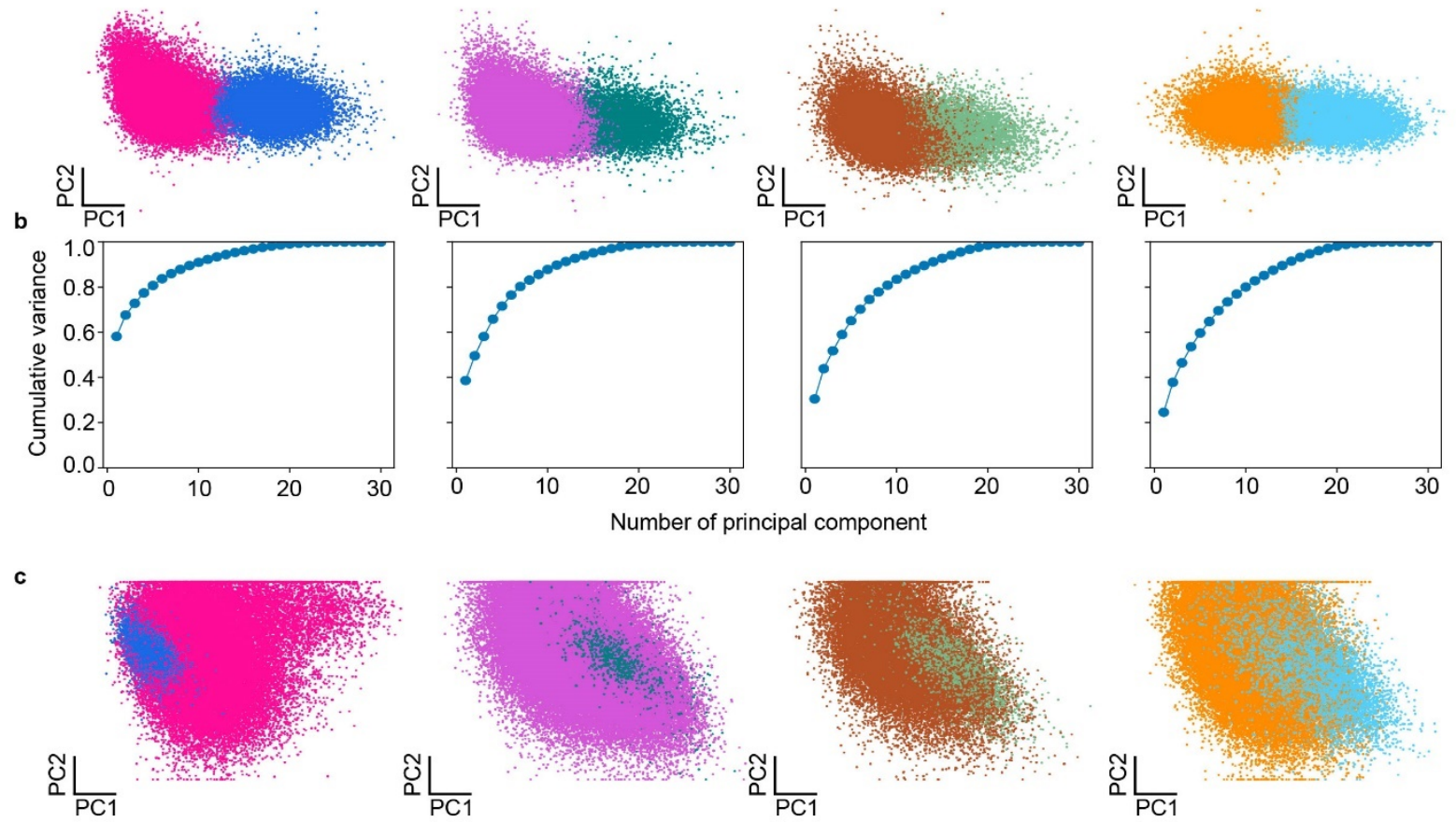

d
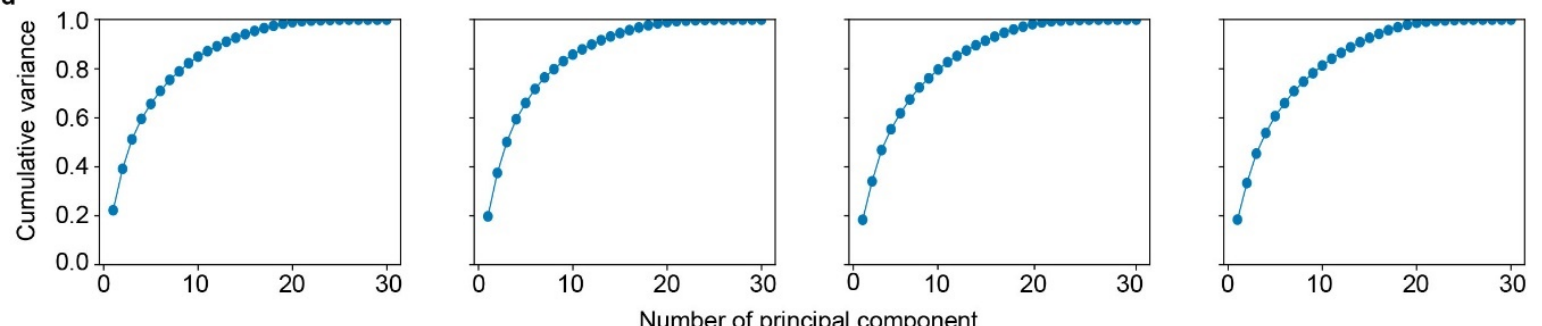

e
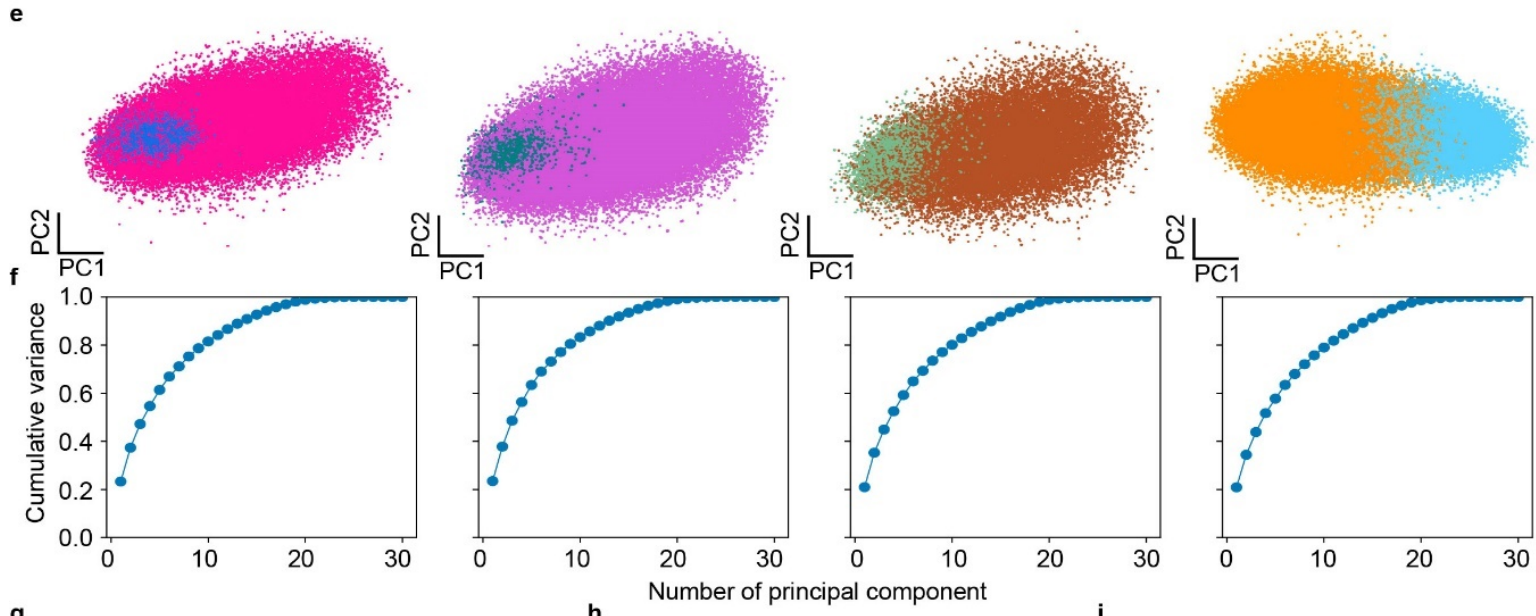

g
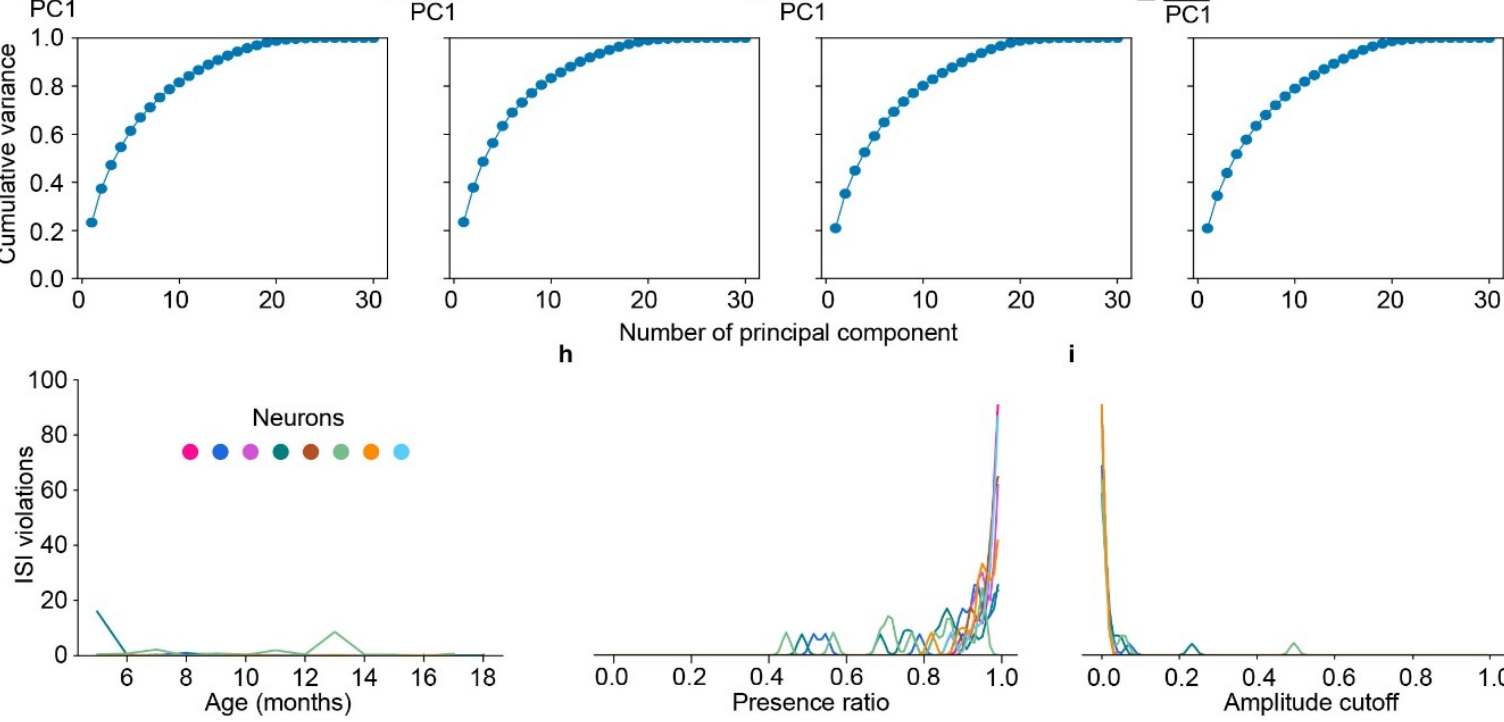

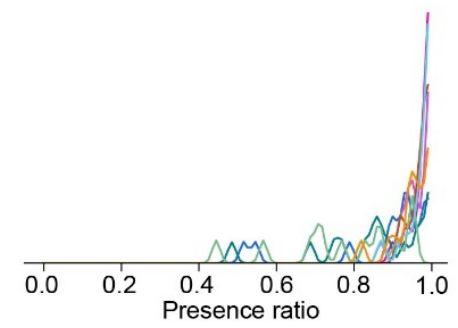

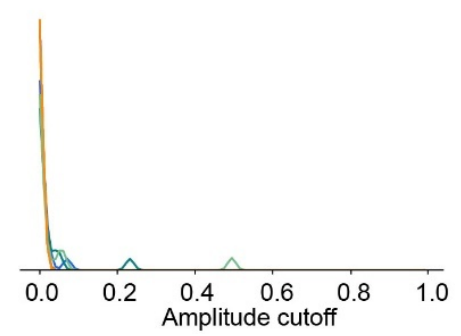


1046 Extended Data Fig. 10 | Quality assessment for PCA embeddings and neuron clusters. a, 1047 Principal Component (PC) embeddings of all recorded spikes without any normalization colored 1048 by neuron. b, Cumulative proportion of variance explained by top principal components of (a). c, 1049 d, Same as (a, b) with prior min-max normalization to scale spikes to $(-1,1)$ range. e, f, Same as 1050 (a, b) with prior standard scaling normalization per spike. Normalization decreases cluster 1051 separability in PC representation and lowers proportion of variance explained by the top two 1052 components by removing amplitude information from spikes. $\mathbf{g}$, Interspike interval (ISI) violations 1053 in the absolute number of spikes calculated over all recordings for each recording month. h, 1054 Presence ratio smoothed density plot over all recording sessions per unit. i, Amplitude cutoff 1055 smoothed density plot over all recording sessions per unit. 